\title{
Markovnikov Selective Hydroboration of Olefins Catalyzed by a Copper N-Heterocyclic Carbene Complex
}

\author{
Tarah A. DiBenedetto, Astrid M. Parsons, William D. Jones* \\ Department of Chemistry, University of Rochester, Rochester, NY 14627 \\ jones@chem.rochester.edu \\ Supporting Information
}

\section{Table of Contents}

1. General information $\quad$ S-2

2. Optimization of hydroboration reaction workup S-4

3. Optimization of electron rich substrates $\quad$ S-5

$\begin{array}{lr}\text { 4. General procedure for stoichiometric studies } & \text { S-7 }\end{array}$

5. Product characterization $\quad$ S-11

6. Large scale reaction $\quad$ S-17

$\begin{array}{ll}\text { 7. Unsuccessful Substrates } & \text { S-19 }\end{array}$

8. GC-MS Data $\quad$ S-20

9. References $\quad$ S-56 


\section{General information}

\subsection{Reagents}

\section{Metal Complexes}

Unless otherwise noted, all organometallic compounds were prepared and handled under a nitrogen atmosphere using standard Schlenk and glovebox techniques.

\section{Ligands}

(1E, 2E)- $N^{1}, N^{2}$-bis(2,6-diisopropylphenyl)ethane-1,2-diimine A was synthesized according to the literature procedure. ${ }^{1}$ 1,3-Bis(2,6-diisopropylphenyl)-1,3-dihydro-2H-imidazol-2-ylidene $\mathbf{B}$ was synthesized according to the literature procedure. ${ }^{2}$

\section{$\underline{\text { Solvents }}$}

Anhydrous tetrahydrofuran (THF) was obtained from an Innovative Technology PS-MD-6 solvent purification system and this was degassed by three freeze-pump-thaw cycles and stored in a nitrogen glovebox. THF- $d_{8}$ and chloroform- $d\left(\mathrm{CDCl}_{3}\right)$ were purchased from Cambridge Isotopes. $\mathrm{CDCl}_{3}$ was used without further purification. THF- $d_{8}$ was degassed by three freeze-pump-thaw cycles and stored over activated $3 \AA$ molecular sieves inside a nitrogen glovebox. Solvents for the preparation of the $N$-heterocyclic carbene salt and the copper precursor were obtained from Fisher Scientific and used without further purification.

\section{Other reagents}

Reagents were used as received from the following sources: 2,4,6-trimethylbenzaldehyde (Combi Blocks), $\alpha, \alpha, \alpha$-trifluorotoluene (Sigma Aldrich), 4-fluorobenzoic acid (Sigma Aldrich), 4,4,5,5tetramethyl-1,3,2-dioxaborolane (Sigma Aldrich), bis(pinacolato)diboron (Combi Blocks), styrene (Sigma Aldrich), 4-cyanostyrene (Alfa Aesar), 4-methylstyrene (Sigma Aldrich), 4trifluoromethylstyrene (Combi Blocks), 4-ethoxystyrene (Combi Blocks), 4-methoxystyrene (Sigma Aldrich), 4-aminostyrene (Combi Blocks), 4-fluorostyrene (Combi Blocks), 2-(4ethenylphenyl)-4,4,5,5-tetramethyl-1,3,2-dioxaborolane (Combi Blocks), 3-methylstyrene (Sigma Aldrich), 3-chlorostyrene (Combi Blocks), 2-methylstyrene (Fluka), 2-chlorostyrene (Combi Blocks), $\alpha$-methylstyrene (Sigma Aldrich), ethynylbenzene (Sigma Aldrich), 1-ethynyl-2methylbenzene (Combi Blocks), 3-ethynylpyridine (Combi Blocks), 1-ethynyl-4-methoxybenzene (Sigma Aldrich), methyl 4-ethynylbenzoate (Combi Blocks), 1,2-dihydronaphthalene (Sigma Aldrich), ethyl acrylate (Sigma Aldrich), 4-vinylpyridine (Sigma Aldrich), triisopropyl(prop-1-yn1-yl)silane (Sigma Aldrich), 1-ethynylcyclohex-1-ene (Sigma Aldrich), 4-bromostyrene (Combi Blocks), 1-ethynyl-4-(trifluoromethyl)benzene (Sigma Aldrich), 1-ethynyl-4-methylbenzene (Sigma Aldrich), 1-chloro-3-ethynylbenzene (Sigma Aldrich), 2,6-diisopropylaniline (TCI), glyoxal (Alfa Aesar), formic acid (Fisher Scientific), paraformaldehyde (Sigma Aldrich), hydrogen chloride $4 \mathrm{M}$ in dioxane (Oakwood), copper(I) oxide (Fisher Scientific), cesium hydroxide monohydrate (Sigma Aldrich) 


\subsection{Methods}

NMR spectroscopy

${ }^{1} \mathrm{H}$ NMR spectra were recorded on 400 and $500 \mathrm{MHz}$ Bruker Avance NMR instruments. NMR chemical shifts are reported in ppm and are referenced to the residual solvent peak $\mathrm{CDCl}_{3}(\delta=$ 7.26) and THF- $d_{8}(\delta=3.58)$. Coupling constants $(J)$ are reported in Hertz.

$\underline{\text { GC-MS analysis }}$

GC-MS spectra were recorded on a Shimadzu QP2010 instrument equipped with a SH-Rxi-5ms column $(15 \mathrm{M} \times 0.25 \mathrm{~mm}$ ID $\times 0.25 \mu$ film $)$. Column flow $=0.7 \mathrm{~mL} / \mathrm{min}$, temperature program: 3 min at $50{ }^{\circ} \mathrm{C}$, then increase at $20{ }^{\circ} \mathrm{C} / \mathrm{min}$ to $235^{\circ} \mathrm{C}$ and hold for $3.5 \mathrm{~min}$.

Chromatography

Chromatography was performed on silica gel (EMD, silica gel 60, particle size 0.040-0.063 mm) using standard flash techniques. Products were visualized by UV.

Elemental analysis

Elemental analyses were obtained from the CENTC Elemental Analysis Facility at the University of Rochester using a Perkin Elmer PerkinElmer 2400 Series II Analyzer. 


\section{Optimization of hydroboration reaction workup}

The general procedure was followed for the setup of the following optimization reactions.

Work-up 1: After $24 \mathrm{~h}$, the reaction was filtered through a celite plug and $\mathrm{CDCl}_{3}(0.6 \mathrm{~mL})$ was used to wash the reaction vial. The washings were filtered through the plug and the crude was analyzed by ${ }^{1} \mathrm{H}$ NMR spectroscopy utilizing 2,4,6-trimethylbenzaldehyde as the internal standard.

Work-up 2: After $24 \mathrm{~h}$, the reaction was filtered through a silica plug and $\mathrm{CDCl}_{3}(0.6 \mathrm{~mL})$ was used to wash the reaction vial. The washings were filtered through the plug and the crude was analyzed by ${ }^{1} \mathrm{H}$ NMR spectroscopy utilizing 2,4,6-trimethylbenzaldehyde as the internal standard.

Work-up 3: After $24 \mathrm{~h}$, the reaction was filtered through a celite plug and the reaction vial was washed with DCM $(5 x 0.5 \mathrm{~mL})$ and the washings were filtered through the plug. The crude reaction was concentrated in vacuo and analyzed by ${ }^{1} \mathrm{H}$ NMR spectroscopy utilizing 2,4,6trimethylbenzaldehyde as the internal standard.

Work-up 4: After $24 \mathrm{~h}$, the reaction was filtered through a silica plug and the reaction vial was washed with $\operatorname{DCM}(5 \mathrm{x} 0.5 \mathrm{~mL})$ and the washings were filtered through the plug. The crude reaction was concentrated in vacuo and analyzed by ${ }^{1} \mathrm{H}$ NMR spectroscopy utilizing 2,4,6trimethylbenzaldehyde as the internal standard.

\section{Table S1. Yields for various workup procedures}

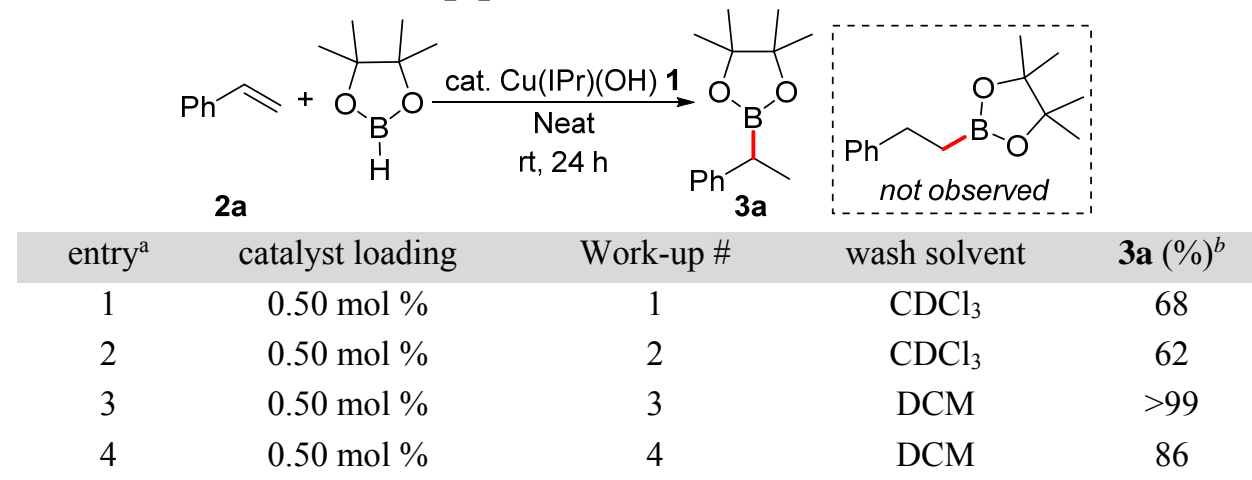

${ }^{a}$ Reaction conditions: alkene $(0.5 \mathrm{mmol})$, HBpin $(0.5 \mathrm{mmol})$, catalyst $1, \mathrm{rt}, 24 \mathrm{~h} .{ }^{b}$ Yield determined via ${ }^{1} \mathrm{H}$ NMR vs 2,4,6-trimethylbenzaldehyde as an internal standard.

Work-up 3 (entry 3) proved to be optimal for analyzing catalytic reactions. 


\section{Optimization of electron rich substrates}

The general reaction setup was followed for the optimization of electron rich substrates. Under mild atom economical (equimolar) conditions, the reaction between para-methoxystyrene and HBpin does not afford a high yield. The use of excess HBpin or $\mathrm{B}_{2} \mathrm{Pin}_{2}$ (entries 3-4) does result in an increase in yield. From our observations, the yield of the borylated product is likely dependent upon how much excess boron is available. When there is no excess boron available (1:1 ratio of alkene to boron), the ${ }^{1} \mathrm{H}$ NMR spectrum and GC-MS chromatogram shows full consumption of the boron species and remaining starting alkene and product.

Table S2. Optimization of conditions<smiles>COc1ccc(C=[C+]C(C)c2ccc(OC)cc2)cc1</smiles>

2g, 1 equiv

\begin{tabular}{c|cccccc} 
Entry $^{a}$ & Catalyst Loading & THF $(\mathrm{mL})$ & Boron Reagent & Boron mmol & Temperature & Yield $^{b}$ \\
\hline 1 & $1 \mathrm{~mol} \%$ & 0.5 & HBpin & $0.5 \mathrm{mmol}$ & $\mathrm{RT}$ & $43 \%$ \\
2 & $1 \mathrm{~mol} \%$ & 0.5 & $\mathrm{~B}_{2}$ Pin $_{2}$ & $0.5 \mathrm{mmol}$ & $\mathrm{RT}$ & $56 \%$ \\
3 & $1 \mathrm{~mol} \%$ & 0.5 & HBpin & $1 \mathrm{mmol}$ & $\mathrm{RT}$ & $49 \%$ \\
4 & $1 \mathrm{~mol} \%$ & 0.5 & $\mathrm{~B}_{2} \mathrm{Pin}_{2}$ & $0.6 \mathrm{mmol}$ & $\mathrm{RT}$ & $69 \%$ \\
5 & $1 \mathrm{~mol} \%$ & -- & HBpin & $0.5 \mathrm{mmol}$ & $\mathrm{RT}$ & $34 \%$ \\
6 & $1.5 \mathrm{~mol} \%$ & -- & HBpin & $0.5 \mathrm{mmol}$ & $\mathrm{RT}$ & $33 \%$ \\
7 & $1 \mathrm{~mol} \%$ & 0.25 & HBpin & $0.5 \mathrm{mmol}$ & $\mathrm{RT}$ & $30 \%$ \\
8 & $1.5 \mathrm{~mol} \%$ & 0.25 & HBpin & $0.5 \mathrm{mmol}$ & $\mathrm{RT}$ & $23 \%$ \\
\hline
\end{tabular}

${ }^{a}$ Reaction conditions: alkene $(0.5 \mathrm{mmol}), \mathrm{HBpin}(0.5 \mathrm{mmol})$, catalyst 1 , THF $(\mathrm{mL}), \mathrm{rt}, 24 \mathrm{~h} .{ }^{b}$ Yield determined via ${ }^{1} \mathrm{H}$ NMR spectroscopy vs 2,4,6-trimethylbenzaldehyde as an internal standard. 


\subsection{Time release optimization of electron poor substrates}

The general reaction setup was followed for the time release studies. Reactions were run for $48 \mathrm{~h}$.

Entry 1: HBpin ( $0.5 \mathrm{mmol}, 1.0$ equiv, $72.6 \mu \mathrm{L})$ was added over 3 portions $(24.2 \mu \mathrm{L}$ per portion). The first portion was added at the beginning of the reaction $(\mathrm{t}=0 \mathrm{~min})$, the next portion was added at $2 \mathrm{~h}$ and the last was added at $24 \mathrm{~h}$. The reaction was then allowed to stir another $24 \mathrm{~h}$ before workup.

Entry 2: HBpin $(0.5 \mathrm{mmol}, 1.0$ equiv, $72.6 \mu \mathrm{L})$ was added at the beginning of the reaction. After $24 \mathrm{~h}$ HBpin $(0.5 \mathrm{mmol}, 1.0$ equiv, $72.6 \mu \mathrm{L})$ was added again for a total of 2 equiv. The reaction was then allowed to stir another $24 \mathrm{~h}$ before workup.

Table S3. Effect of Boron loading on products

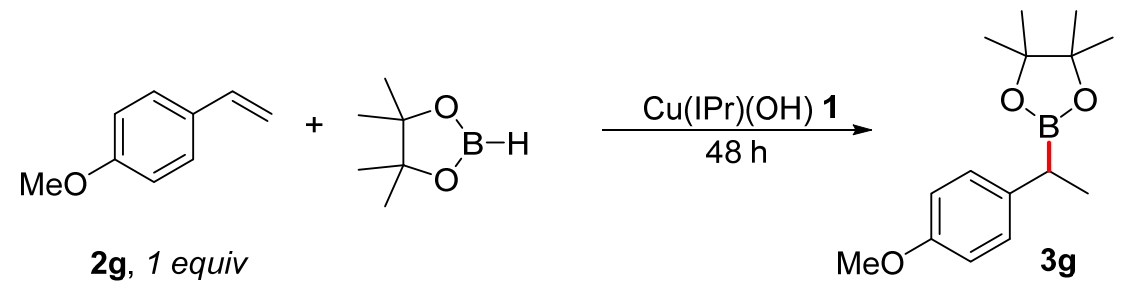

2g, 1 equiv

\begin{tabular}{ccccc} 
Entry $^{a}$ & Catalyst Loading & Boron mmol & Temperature & $(\mathbf{2 g}: 3 \mathbf{g})^{b}$ \\
\hline 1 & $1 \mathrm{~mol} \mathrm{\%}$ & $0.5 \mathrm{mmol}$ & RT & $(1.29: 1)$ \\
2 & $1 \mathrm{~mol} \mathrm{\%}$ & $1 \mathrm{mmol}$ & RT & $(1: 1.38)$
\end{tabular}

${ }^{a}$ Reaction conditions: alkene $(0.5 \mathrm{mmol})$, HBpin $(0.5 \mathrm{mmol})$, catalyst 1 , rt, $48 \mathrm{~h} .{ }^{b}$ Ratio of (2:3) determined via ${ }^{1} \mathrm{H}$ NMR spectroscopy vs 2,4,6-trimethylbenzaldehyde as an internal standard. 


\section{General procedure for stoichiometric studies}

$\underline{\text { Stoichiometric reaction of } 1 \text { with HBpin in THF- } d 8}$

$$
\underset{1,1 \text { equiv }}{\mathrm{Cu}(\mathrm{IPr})(\mathrm{OH})}+\underset{\substack{\text { 1 equiv } \\ \text { 1, Bpin }}}{\stackrel{\mathrm{THF}_{-d 8}}{\longrightarrow}} \underset{\mathbf{4}}{\mathrm{Cu}(\mathrm{IPr})(\mathrm{H})}
$$

In a nitrogen-filled glovebox: an oven-dried J-Young NMR test tube was charged with $\mathrm{Cu}(\mathrm{IPr})(\mathrm{OH})(0.05 \mathrm{mmol}, 1.0$ equiv, $23.5 \mathrm{mg})$ and THF-d $\mathrm{d}_{8}(0.6 \mathrm{~mL})$ resulting in a colorless solution. Next, HBpin $(0.05 \mathrm{mmol}, 1.0$ equiv, $6.5 \mathrm{mg}, 7.3 \mu \mathrm{L})$ was added and upon mixing the solution immediately became bright yellow in color. The tube was capped and brought out of the glovebox and the ${ }^{1} \mathrm{H}$ NMR spectrum was recorded as soon as possible (ca. $5 \mathrm{~min}$ ) thereafter.

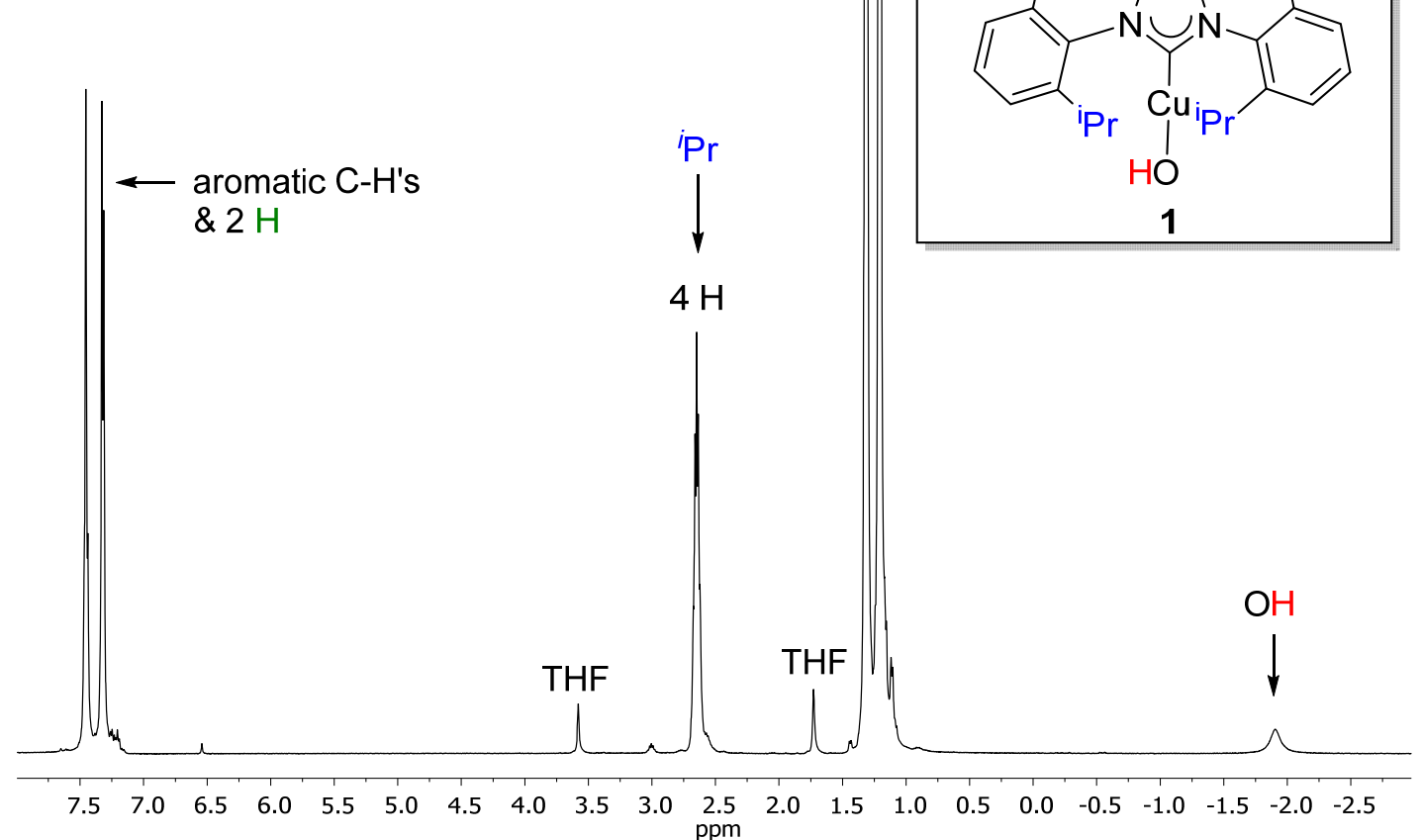

Figure S1. ${ }^{1} \mathrm{H}$ NMR spectra of 1 in THF-d8. 


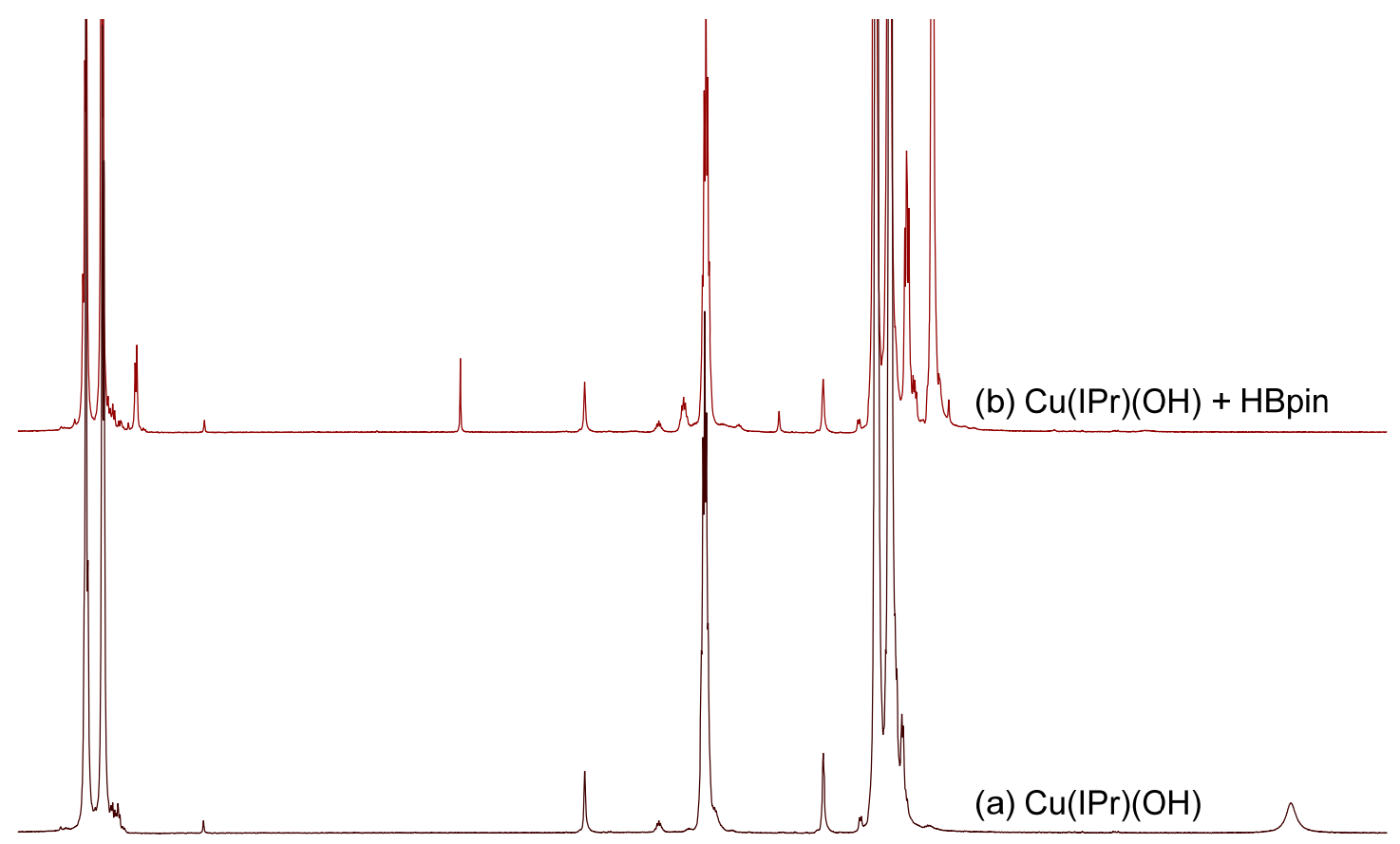

$\begin{array}{lllllllllllllllllllll}7.5 & 7.0 & 6.5 & 6.0 & 5.5 & 5.0 & 4.5 & 4.0 & 3.5 & 3.0 & \begin{array}{c}2.5 \\ \mathrm{ppm}\end{array} & 2.0 & 1.5 & 1.0 & 0.5 & 0.0 & -0.5 & -1.0 & -1.5 & -2.0 & -2.5\end{array}$

Figure S2. ${ }^{1} \mathrm{H}$ NMR spectra in THF- $d_{8}$ of the stoichiometric reaction of 1 with HBpin. (a) before the addition of HBpin. (b) after the addition of HBpin (ca. 5 min). 


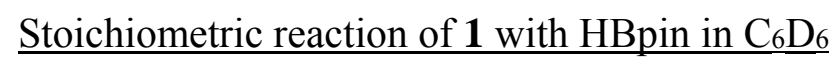

$$
\underset{\mathbf{1}, 1 \text { equiv }}{\mathrm{Cu}(\mathrm{IPr})(\mathrm{OH})+\underset{1 \text { equiv }}{\mathrm{H}-\text { Bpin }} \stackrel{\mathrm{C}_{6} \mathrm{D}_{6}}{\longrightarrow} \mathrm{Cu}(\mathrm{IPr})(\mathrm{H})}
$$

In a nitrogen-filled glovebox: an oven-dried J-Young NMR test tube was charged with $\mathrm{Cu}(\mathrm{IPr})(\mathrm{OH})(0.05 \mathrm{mmol}, 1.0$ equiv, $23.5 \mathrm{mg})$ and $\mathrm{C}_{6} \mathrm{D}_{6}(0.6 \mathrm{~mL})$ resulting in a colorless solution. Next, HBpin $(0.05 \mathrm{mmol}, 1.0$ equiv, $6.5 \mathrm{mg}, 7.3 \mu \mathrm{L})$ was added and upon mixing the solution immediately became bright yellow in color. The tube was capped and brought out of the glovebox and the ${ }^{1} \mathrm{H}$ NMR spectrum was recorded as soon as possible $(\mathrm{ca} .5 \mathrm{~min})$ thereafter.

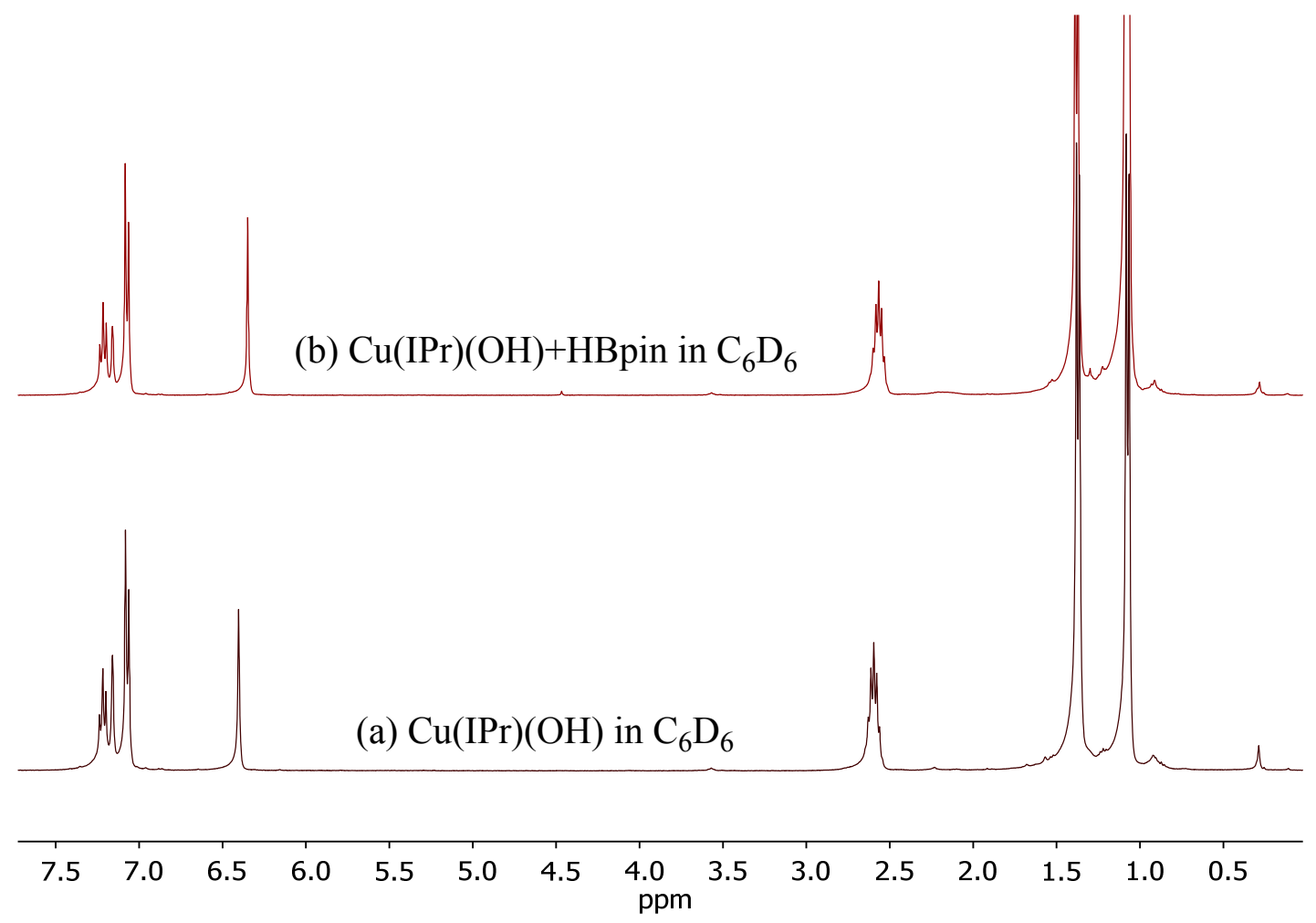

Figure S3. ${ }^{1} \mathrm{H}$ NMR spectra in $\mathrm{C}_{6} \mathrm{D}_{6}$ of the stoichiometric reaction of 1 with HBpin. (a) before the addition of HBpin. (b) after the addition of HBpin (ca. $5 \mathrm{~min}$ ). 
$\underline{\text { Stoichiometric reaction of } \mathbf{4} \text { with HBpin and alkene 2a }}$

$$
\underset{4}{\mathrm{Cu}(\mathrm{IPr})(\mathrm{H})}+\underset{1 \text { equiv }}{\mathrm{H}-\text { Bpin }}+\underset{\mathbf{2} \mathbf{a}, 1 \text { equiv }}{\mathrm{Ph}} \stackrel{\mathrm{THF}-\mathrm{d}_{8}}{\longrightarrow} \mathbf{3 a}
$$

In a nitrogen-filled glovebox: an oven-dried J-Young NMR test tube was charged with $\mathrm{Cu}(\mathrm{IPr})(\mathrm{OH})(0.05 \mathrm{mmol}, 1$ equiv, $23.5 \mathrm{mg})$ and THF- $d_{8}(0.6 \mathrm{~mL})$ resulting in a colorless solution. Next, HBpin $(0.05 \mathrm{mmol}, 1$ equiv, $6.5 \mathrm{mg}, 7.3 \mu \mathrm{L})$ was added and upon mixing the solution immediately became bright yellow in color. The bright yellow solution indicates the formation of 4. Next, 2a (0.05 mmol, 1 equiv, $5.2 \mathrm{mg}, 5.7 \mu \mathrm{L})$ and $\mathrm{HBpin}(0.05 \mathrm{mmol}, 1$ equiv, $6.5 \mathrm{mg}, 7.3 \mu \mathrm{L})$ were quickly added and the tube was capped and brought out of the glovebox and reaction progress was monitored by ${ }^{1} \mathrm{H}$ NMR spectroscopy.

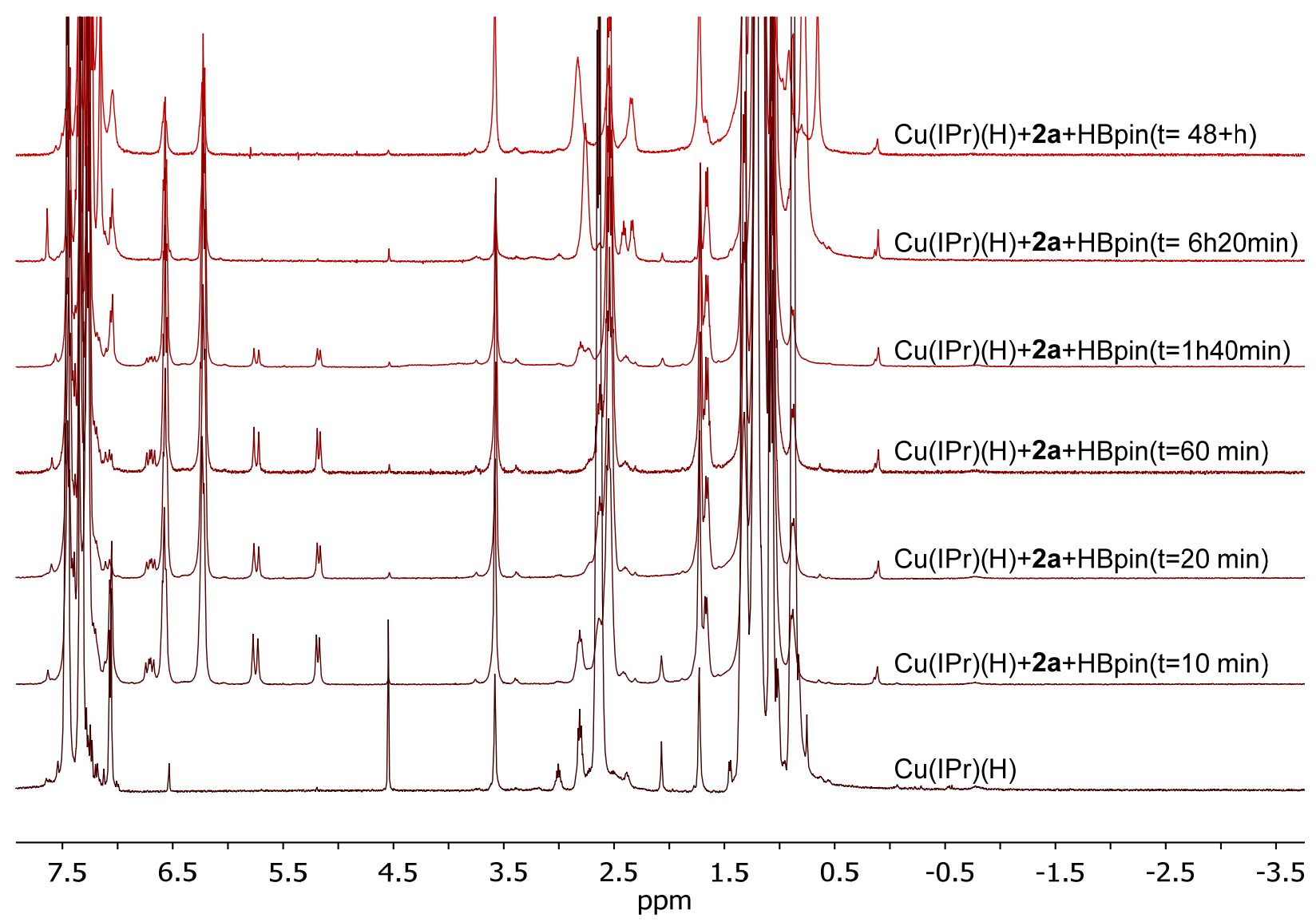

Figure S4. ${ }^{1} \mathrm{H}$ NMR spectra in THF- $d_{8}$ of the stoichiometric reaction of $\mathbf{4}$ with HBpin and $2 \mathbf{2}$. 


\section{Product characterization}<smiles>CC(Br)c1ccccc1</smiles>

3a

\section{4,4,5,5-tetramethyl-2-(1-phenylethyl)-1,3,2-dioxaborolane}

The general procedure for hydroboration was followed with styrene $(0.5 \mathrm{mmol}, 1.0$ equiv, 57.5 $\mu \mathrm{L})$ and 4,4,5,5-tetramethyl-1,3,2-dioxaborolane (0.5 mmol, 1.0 equiv, $72.6 \mu \mathrm{L})$. Spectroscopic data matches the reported literature. ${ }^{3}$<smiles>CC(c1ccccc1)c1ccc(Cl)cc1</smiles>

\section{2-(1-(4-chlorophenyl)ethyl)-4,4,5,5-tetramethyl-1,3,2-dioxaborolane}

The general procedure for hydroboration was followed with 4-chlorostyrene ( $0.5 \mathrm{mmol}, 1.0$ equiv, $60.0 \mu \mathrm{L})$ and 4,4,5,5-tetramethyl-1,3,2-dioxaborolane $(0.5 \mathrm{mmol}, 1.0$ equiv, $72.6 \mu \mathrm{L})$. Spectroscopic data matches the reported literature. ${ }^{4}$<smiles>CC(c1ccccc1)c1ccc(C#N)cc1</smiles>

\section{4-(1-(4,4,5,5-tetramethyl-1,3,2-dioxaborolan-2-yl)ethyl)benzonitrile}

The general procedure for hydroboration was followed with 4-cyanostyrene ( $0.5 \mathrm{mmol}, 1.0$ equiv, $60.0 \mu \mathrm{L})$ and 4,4,5,5-tetramethyl-1,3,2-dioxaborolane (0.5 mmol, 1.0 equiv, $72.6 \mu \mathrm{L})$. Spectroscopic data matches the reported literature. ${ }^{5}$<smiles>CC(c1ccccc1)c1ccc(C(F)(F)F)cc1</smiles>

\section{4,4,5,5-tetramethyl-2-(1-(4-(trifluoromethyl)phenyl)ethyl)-1,3,2-dioxaborolane}

The general procedure for hydroboration was followed with 4-trifluoromethylstyrene $(0.5 \mathrm{mmol}$, 1.0 equiv, $73.9 \mu \mathrm{L})$ and 4,4,5,5-tetramethyl-1,3,2-dioxaborolane (0.5 mmol, 1.0 equiv, $72.6 \mu \mathrm{L})$. Spectroscopic data matches the reported literature. ${ }^{4}$ 
<smiles>CC(c1ccccc1)c1ccc(F)cc1</smiles>

\section{2-(1-(4-fluorophenyl)ethyl)-4,4,5,5-tetramethyl-1,3,2-dioxaborolane}

The general procedure for hydroboration was followed with 4-fluorostyrene $(0.5 \mathrm{mmol}, 1.0$ equiv, $59.6 \mu \mathrm{L})$ and 4,4,5,5-tetramethyl-1,3,2-dioxaborolane (0.5 mmol, 1.0 equiv, $72.6 \mu \mathrm{L})$. Spectroscopic data matches the reported literature. ${ }^{6}$<smiles>Cc1ccc(C(C)c2ccccc2)cc1</smiles>

\section{4,4,5,5-tetramethyl-2-(1-(p-tolyl)ethyl)-1,3,2-dioxaborolane}

The general procedure for hydroboration was followed with 4-methylstyrene ( $0.5 \mathrm{mmol}, 1.0$ equiv, $65.9 \mu \mathrm{L})$ and 4,4,5,5-tetramethyl-1,3,2-dioxaborolane (0.5 mmol, 1.0 equiv, $72.6 \mu \mathrm{L})$. Spectroscopic data matches the reported literature. ${ }^{7}$<smiles>COc1ccc(C(C)c2ccccc2)cc1</smiles>

\section{2-(1-(4-methoxyphenyl)ethyl)-4,4,5,5-tetramethyl-1,3,2-dioxaborolane}

The general procedure for hydroboration was followed with 4-methoxystyrene $(0.5 \mathrm{mmol}, 1.0$ equiv, $66.5 \mu \mathrm{L})$ and 4,4,5,5-tetramethyl-1,3,2-dioxaborolane ( $0.5 \mathrm{mmol}, 1.0$ equiv, $72.6 \mu \mathrm{L})$. Spectroscopic data matches the reported literature. ${ }^{4}$<smiles>CC(c1ccccc1)c1ccc(Br)cc1</smiles>

\section{4,4,5,5-tetramethyl-2-(4-(1-(4,4,5,5-tetramethyl-1,3,2-dioxaborolan-2-yl)ethyl)phenyl)-1,3,2-} dioxaborolane

The general procedure for hydroboration was followed with 2-(4-ethenylphenyl)-4,4,5,5tetramethyl-1,3,2-dioxaborolane $(0.5 \mathrm{mmol}, 1.0$ equiv, $0.115 \mathrm{~g})$ and 4,4,5,5-tetramethyl-1,3,2dioxaborolane $(0.5 \mathrm{mmol}, 1.0$ equiv, $72.6 \mu \mathrm{L})$. Spectroscopic data matches the reported literature. ${ }^{8}$ 
<smiles>Cc1cccc(C(C)c2ccccc2)c1</smiles>

3i

\section{4,4,5,5-tetramethyl-2-(1-(m-tolyl)ethyl)-1,3,2-dioxaborolane}

The general procedure for hydroboration was followed with 3-methylstyrene $(0.5 \mathrm{mmol}, 1.0$ equiv, $66.4 \mu \mathrm{L})$ and 4,4,5,5-tetramethyl-1,3,2-dioxaborolane (0.5 mmol, 1.0 equiv, $72.6 \mu \mathrm{L})$. Spectroscopic data matches the reported literature. ${ }^{5}$<smiles>CC(Br)c1cccc(Cl)c1</smiles>

3j

\section{2-(1-(3-chlorophenyl)ethyl)-4,4,5,5-tetramethyl-1,3,2-dioxaborolane}

The general procedure for hydroboration was followed with 3 -chlorostyrene $(0.5 \mathrm{mmol}, 1.0$ equiv, $63.6 \mu \mathrm{L})$ and 4,4,5,5-tetramethyl-1,3,2-dioxaborolane (0.5 mmol, 1.0 equiv, $72.6 \mu \mathrm{L})$. Spectroscopic data matches the reported literature. ${ }^{9}$<smiles>Cc1ccccc1C(C)Br</smiles>

\section{4,4,5,5-tetramethyl-2-(1-(o-tolyl)ethyl)-1,3,2-dioxaborolane}

The general procedure for hydroboration was followed with 2-methylstyrene $(0.5 \mathrm{mmol}, 1.0$ equiv, $64.6 \mu \mathrm{L})$ and 4,4,5,5-tetramethyl-1,3,2-dioxaborolane (0.5 mmol, 1.0 equiv, $72.6 \mu \mathrm{L})$. Spectroscopic data matches the reported literature. ${ }^{4}$<smiles>CC(c1ccccc1)c1ccccc1Cl</smiles>

\section{2-(1-(2-chlorophenyl)ethyl)-4,4,5,5-tetramethyl-1,3,2-dioxaborolane}

The general procedure for hydroboration was followed with 2-chlorostyrene $(0.5 \mathrm{mmol}, 1.0$ equiv, $64.2 \mu \mathrm{L})$ and 4,4,5,5-tetramethyl-1,3,2-dioxaborolane (0.5 mmol, 1.0 equiv, $72.6 \mu \mathrm{L})$. Spectroscopic data matches the reported literature. ${ }^{4}$ 
<smiles>Brc1ccccc1</smiles>

$3 \mathrm{~m}$

\section{4,4,5,5-tetramethyl-2-(1,2,3,4-tetrahydronaphthalen-1-yl)-1,3,2-dioxaborolane}

The general procedure for hydroboration was followed with 1,2-dihydronaphthalene $(0.5 \mathrm{mmol}$, 1.0 equiv, $65.3 \mu \mathrm{L})$ and 4,4,5,5-tetramethyl-1,3,2-dioxaborolane ( $0.5 \mathrm{mmol}, 1.0$ equiv, $72.6 \mu \mathrm{L})$. Spectroscopic data matches the reported literature. ${ }^{10}$<smiles>CCOC(=O)C(C)Br</smiles>

$3 n$

ethyl 2-(4,4,5,5-tetramethyl-1,3,2-dioxaborolan-2-yl)propanoate

The general procedure for hydroboration was followed with ethyl acrylate $(0.5 \mathrm{mmol}, 1.0$ equiv, $54.5 \mu \mathrm{L})$ and 4,4,5,5-tetramethyl-1,3,2-dioxaborolane (0.5 mmol, 1.0 equiv, $72.6 \mu \mathrm{L})$. Spectroscopic data matches the reported literature. ${ }^{11}$<smiles>CC(C)(Br)c1ccccc1</smiles>

30

\section{4,4,5,5-tetramethyl-2-(2-phenylpropan-2-yl)-1,3,2-dioxaborolane}

The general procedure for hydroboration was followed with $\alpha$-methylstyrene $(0.5 \mathrm{mmol}, 1.0$ equiv, $65.0 \mu \mathrm{L})$ and 4,4,5,5-tetramethyl-1,3,2-dioxaborolane ( $0.5 \mathrm{mmol}, 1.0$ equiv, $72.6 \mu \mathrm{L})$.

NOTE: no borylation (branched or linear) of the alkene was observed by NMR spectroscopy, but some $(<5 \%)$ branched isomer was seen by GCMS.<smiles>C=C(Br)c1ccccc1</smiles>

$3 p$

\section{4,4,5,5-tetramethyl-2-(1-phenylvinyl)-1,3,2-dioxaborolane}

The general procedure for hydroboration was followed with ethynylbenzene $(0.5 \mathrm{mmol}, 1.0$ equiv, $54.9 \mu \mathrm{L})$ and 4,4,5,5-tetramethyl-1,3,2-dioxaborolane (0.5 mmol, 1.0 equiv, $72.6 \mu \mathrm{L})$. Spectroscopic data matches the reported literature. ${ }^{12}$ 
<smiles>C=C(Br)c1ccccc1</smiles>

$3 q$

\section{4,4,5,5-tetramethyl-2-(1-(o-tolyl)vinyl)-1,3,2-dioxaborolane}

The general procedure for hydroboration was followed with 1-ethynyl-2-methylbenzene $(0.5$ mmol, 1.0 equiv, $63.0 \mu \mathrm{L}$ ) and 4,4,5,5-tetramethyl-1,3,2-dioxaborolane ( $0.5 \mathrm{mmol}, 1.0$ equiv, 72.6 $\mu \mathrm{L})$. Spectroscopic data matches the reported literature. ${ }^{13}$<smiles>C=C(c1ccccc1)c1cccnc1</smiles>

\section{3-(1-(4,4,5,5-tetramethyl-1,3,2-dioxaborolan-2-yl)vinyl)pyridine}

The general procedure for hydroboration was followed with 3-ethynylpyridine ( $0.5 \mathrm{mmol}, 1.0$ equiv, $0.052 \mathrm{~g})$ and 4,4,5,5-tetramethyl-1,3,2-dioxaborolane $(0.5 \mathrm{mmol}, 1.0$ equiv, $72.6 \mu \mathrm{L})$.

${ }^{1} \mathrm{H}$ NMR (400 MHz, Chloroform-d) $\delta 8.57(\mathrm{~s}, 1 \mathrm{H}), 8.41-8.38(\mathrm{~m}, 1 \mathrm{H}), 7.71-7.65(\mathrm{~m}, 1 \mathrm{H}), 7.26$ $(\mathrm{d}, J=18.4 \mathrm{~Hz}, 1 \mathrm{H}), 7.18-7.11(\mathrm{~m}, 1 \mathrm{H}), 6.14(\mathrm{~d}, J=18.5 \mathrm{~Hz}, 1 \mathrm{H}), 1.20$ (s, 12H).

GC-MS: $231(\mathrm{M}+)$

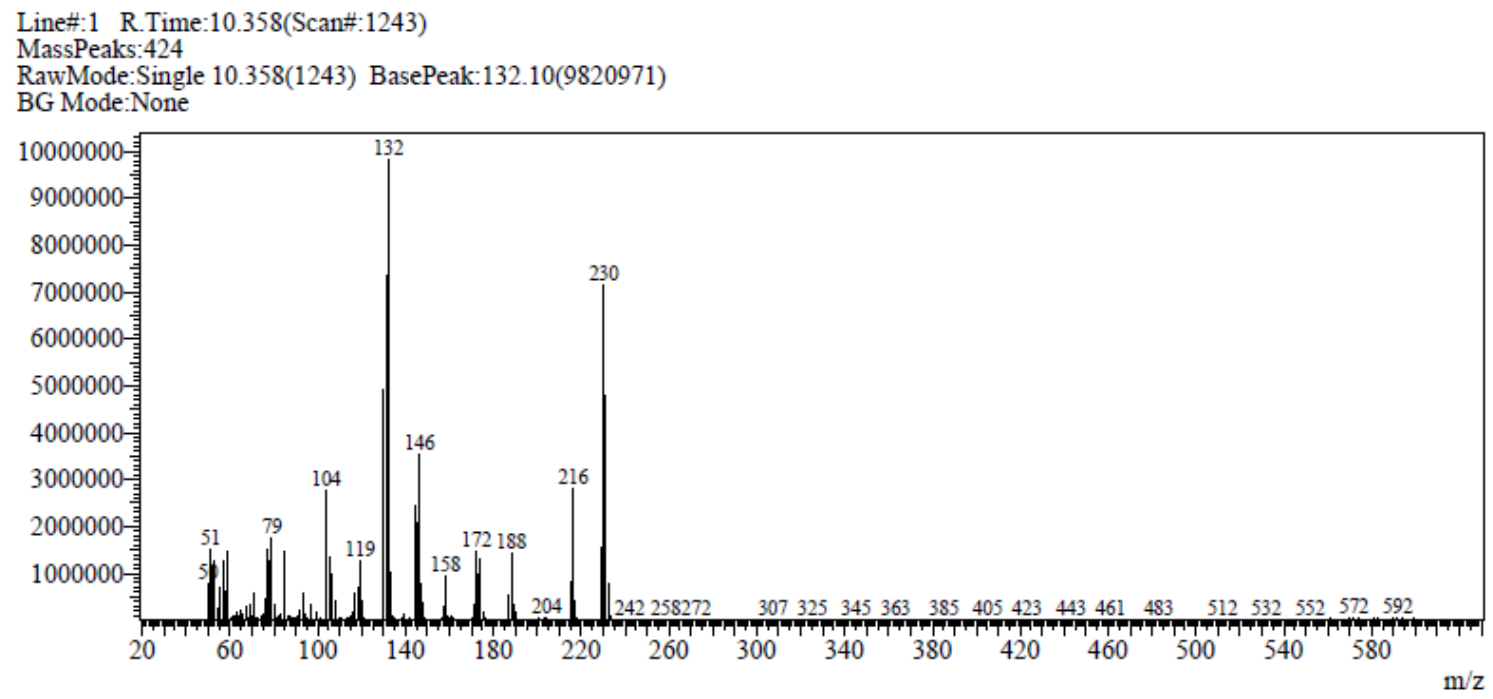

Figure S5. GC-MS chromatogram of borylated product 3r. 
<smiles>C=C(Cc1ccccc1)c1ccc(OC)cc1</smiles>

\section{2-(1-(4-methoxyphenyl)vinyl)-4,4,5,5-tetramethyl-1,3,2-dioxaborolane}

The general procedure for hydroboration was followed with 1-ethynyl-4-methoxybenzene $(0.5$ mmol, 1.0 equiv, $64.8 \mu \mathrm{L}$ ) and 4,4,5,5-tetramethyl-1,3,2-dioxaborolane ( $0.5 \mathrm{mmol}, 1.0$ equiv, 72.6 $\mu \mathrm{L})$. Spectroscopic data matches the reported literature. ${ }^{12}$

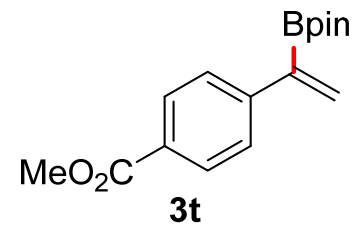

\section{methyl 4-(1-(4,4,5,5-tetramethyl-1,3,2-dioxaborolan-2-yl)vinyl)benzoate}

The general procedure for hydroboration was followed with methyl 4-ethynylbenzoate $(0.5 \mathrm{mmol}$, 1.0 equiv, $0.080 \mathrm{~g})$ and 4,4,5,5-tetramethyl-1,3,2-dioxaborolane (0.5 mmol, 1.0 equiv, $72.6 \mu \mathrm{L})$. Spectroscopic data matches the reported literature. ${ }^{14}$

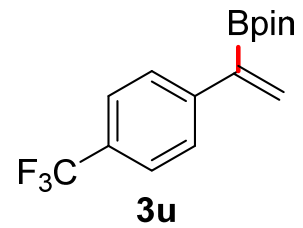

\section{4,4,5,5-tetramethyl-2-(1-(4-(trifluoromethyl)phenyl)vinyl)-1,3,2-dioxaborolane}

The general procedure for hydroboration was followed with 1-ethynyl-4-(trifluoromethyl)benzene ( $0.5 \mathrm{mmol}, 1.0$ equiv, $81.6 \mu \mathrm{L})$ and 4,4,5,5-tetramethyl-1,3,2-dioxaborolane ( $0.5 \mathrm{mmol}, 1.0$ equiv, $72.6 \mu \mathrm{L})$. Spectroscopic data matches the reported literature. ${ }^{15}$<smiles>C=C(Br)c1ccc(C)cc1</smiles>

3v

\section{4,4,5,5-tetramethyl-2-(1-(p-tolyl)vinyl)-1,3,2-dioxaborolane}

The general procedure for hydroboration was followed with 1-ethynyl-4-methylbenzene $(0.5$ mmol, 1.0 equiv, $63.4 \mu \mathrm{L}$ ) and 4,4,5,5-tetramethyl-1,3,2-dioxaborolane ( $0.5 \mathrm{mmol}, 1.0$ equiv, 72.6 $\mu \mathrm{L})$. Spectroscopic data matches the reported literature. ${ }^{13}$<smiles>C=C(Br)c1cccc(Br)c1</smiles>

\section{2-(1-(3-chlorophenyl)vinyl)-4,4,5,5-tetramethyl-1,3,2-dioxaborolane}

The general procedure for hydroboration was followed with 1-chloro-3-ethynylbenzene $(0.5$ mmol, 1.0 equiv, $61.6 \mu \mathrm{L}$ ) and 4,4,5,5-tetramethyl-1,3,2-dioxaborolane ( $0.5 \mathrm{mmol}, 1.0$ equiv, 72.6 $\mu \mathrm{L})$. Spectroscopic data matches the reported literature. ${ }^{13}$ 


\section{Large scale reaction}

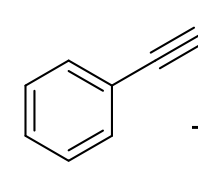

1 equiv

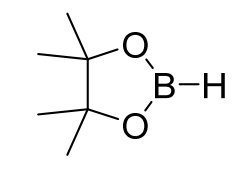

1 equiv $\underset{0.5 \mathrm{~mol} \% \mathrm{Cu}(\mathrm{IPr})(\mathrm{OH}) 1}{\longrightarrow}$

neat, rt, $24 \mathrm{~h}$

$96 \%$ yield

General procedure for hydroboration was followed for the large scale reaction, with slight modifications (vial size, workup). In a glovebox, an oven-dried 2-dram vial containing a stir bar was charged with $\mathrm{Cu}(\mathrm{IPr})(\mathrm{OH})(0.50 \mathrm{~mol} \%, 0.05$ equiv), phenylacetylene $(5.9 \mathrm{mmol}, 1.0$ equiv, $0.648 \mathrm{~mL})$, and HBpin $(5.9 \mathrm{mmol}, 1.0$ equiv, $0.856 \mathrm{~mL})$. Note the reaction was highly exothermic and was allowed to cool enough to touch the vial before the reaction was capped and removed from the glovebox and stirred at room temperature for $24 \mathrm{~h}$. The reaction was then filtered through a celite $(5 \mathrm{~mL}) /$ silica $(5 \mathrm{~mL})$ plug. The vial was washed with an eluent of $8: 2$ hexanes/ethyl acetate and added to the plug. The plug was then washed with $350 \mathrm{~mL}$ of the $8: 2$ hexanes/ethyl acetate eluent. The combined washings were concentrated in vacuo and dried on a vacuum line to afford $3 p\left(1.30 \mathrm{~g}, 96 \%\right.$ yield). Spectroscopic data matches the reported data. ${ }^{12}$

${ }^{1} \mathrm{H}$ NMR (400 MHz, Chloroform- $d$ ) $\delta 7.49$ (d, $\left.J=7.0 \mathrm{~Hz}, 2 \mathrm{H}\right), 7.40$ (d, $\left.J=18.5 \mathrm{~Hz}, 1 \mathrm{H}\right), 7.36$ 7.29 (m, 2H), 6.19 (s, 1H), $6.15(\mathrm{~s}, 1 \mathrm{H}) 1.32(\mathrm{~s}, 12 \mathrm{H})$.

${ }^{13} \mathrm{C}$ NMR (101 MHz, Chloroform-d) $\delta$ 149.65, 137.62, 129.03, 128.71, 127.20, 83.49, 24.96. 


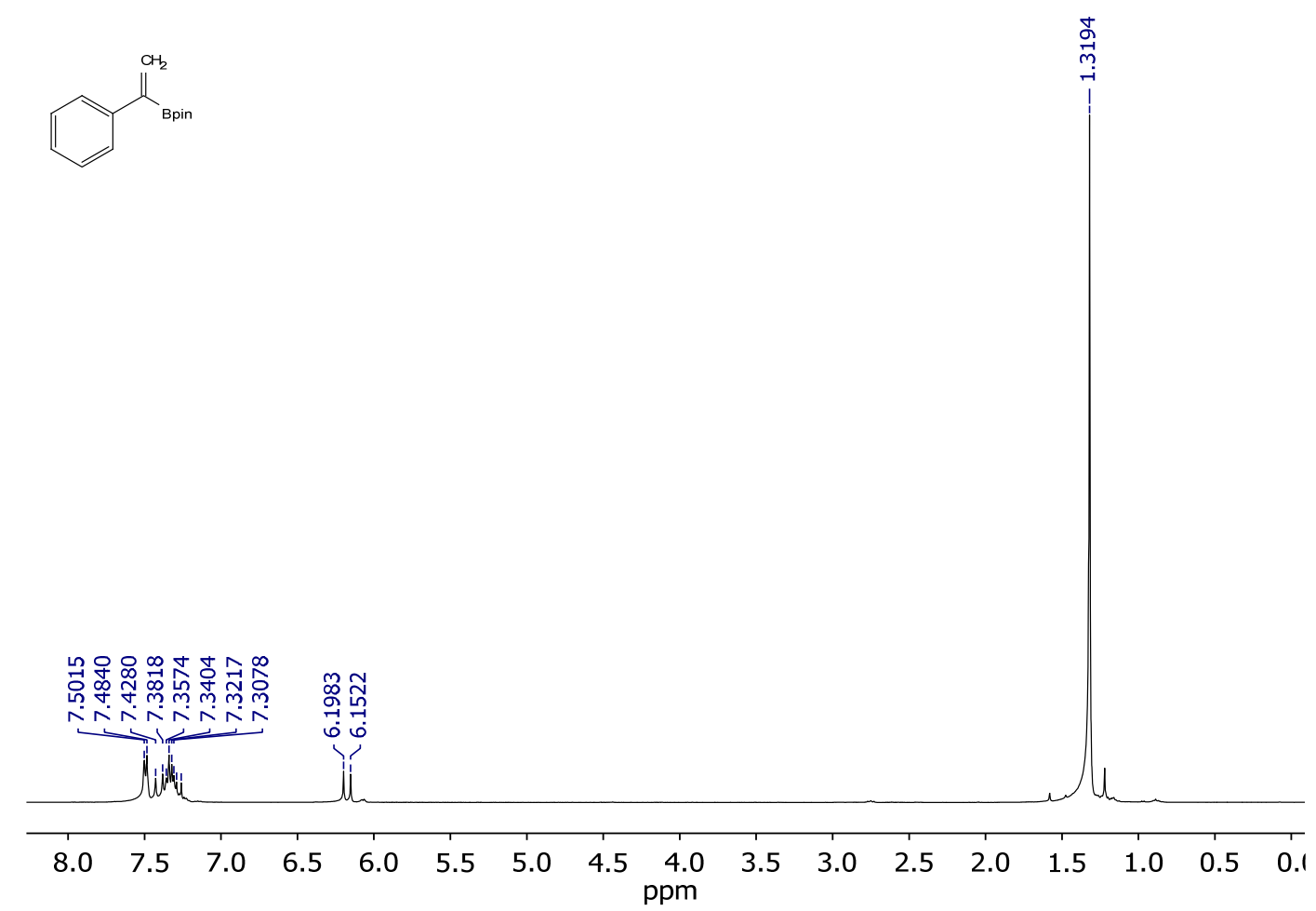

Figure S6. ${ }^{1} \mathrm{H}$ NMR spectrum of borylated product 3p.

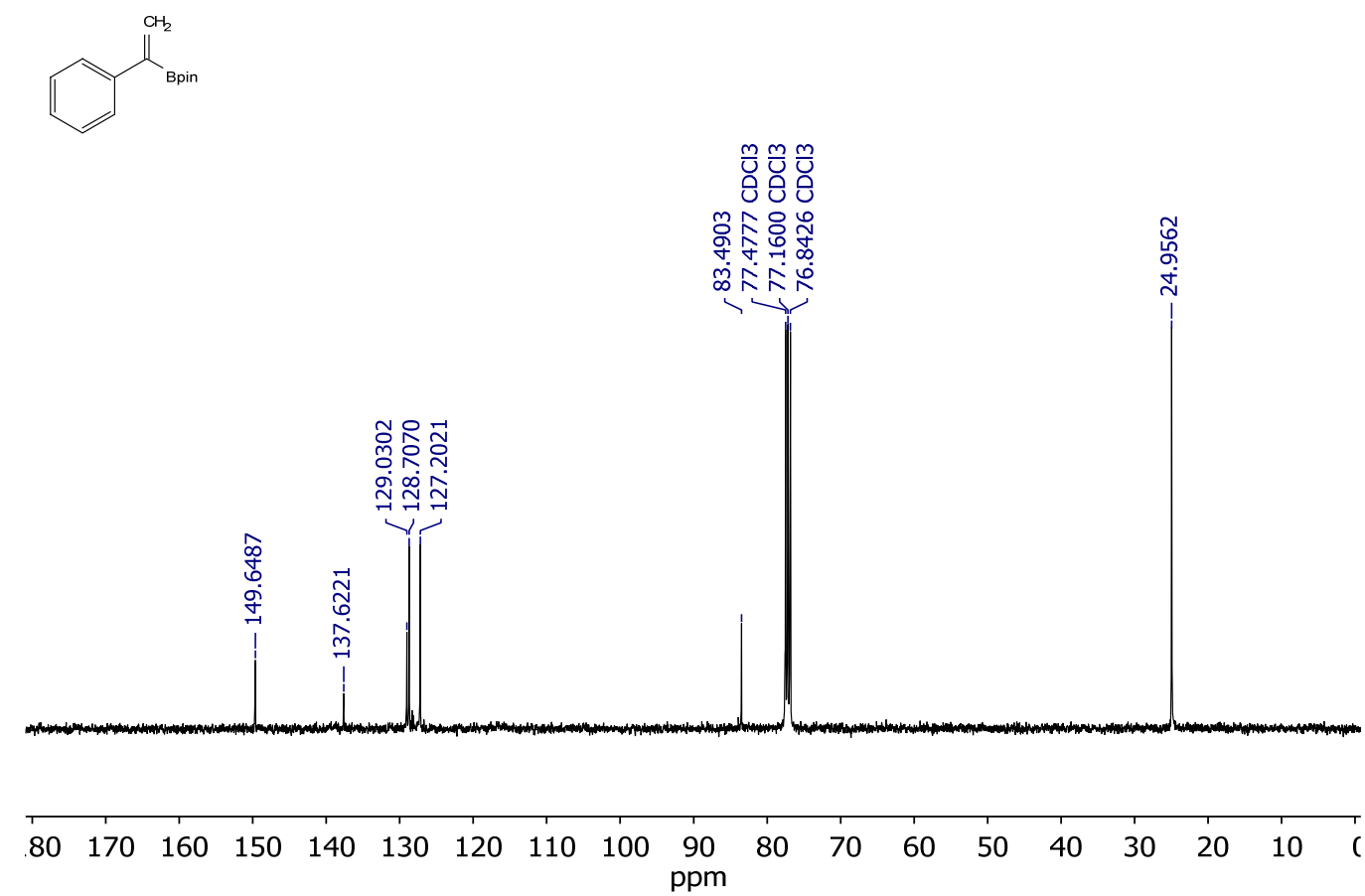

Figure S7. ${ }^{13} \mathrm{C}\left\{{ }^{1} \mathrm{H}\right\}$ NMR spectrum of borylated product $\mathbf{3 p}$. 


\section{Unsuccessful substrates}

The substrates shown below were run following the general procedure for hydroboration. Note that the substrates that ended up in this table showed less than a $25 \%$ yield by ${ }^{1} \mathrm{H} \mathrm{NMR}$ spectroscopy using 2,4,6-trimethylbenzaldehyde as the internal standard. For 4-bromostyrene and 1-ethynylcyclohex-1-ene, two hydroboration products were observed. In the case of 4bromostyrene we saw competition for borylation at both the alkene and the bromide, and for 1ethynylcyclohex-1-ene we saw competition for borylation at both the alkene and alkyne. The unselective borylation made the determination of yields by ${ }^{1} \mathrm{H}$ NMR spectroscopy unreliable; the GCMS chromatograms showed many products.

Table S4. Unsuccessful substrates

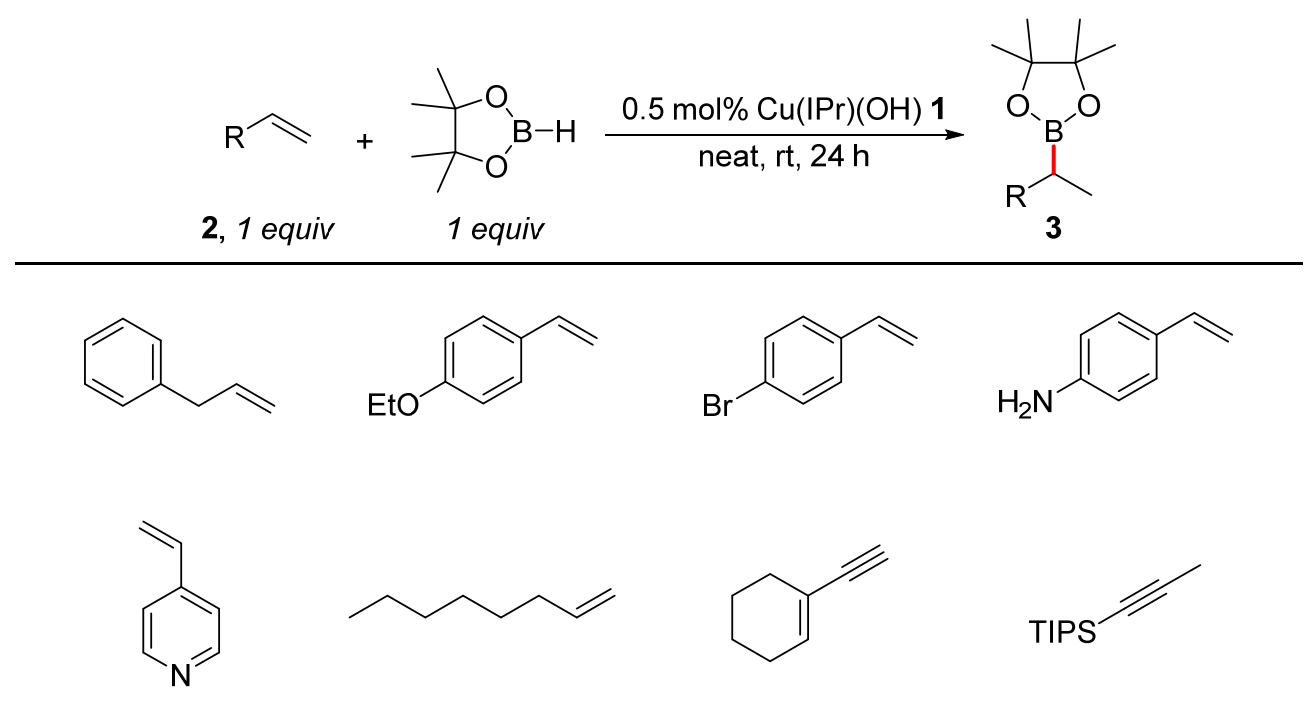




\section{GCMS Data}

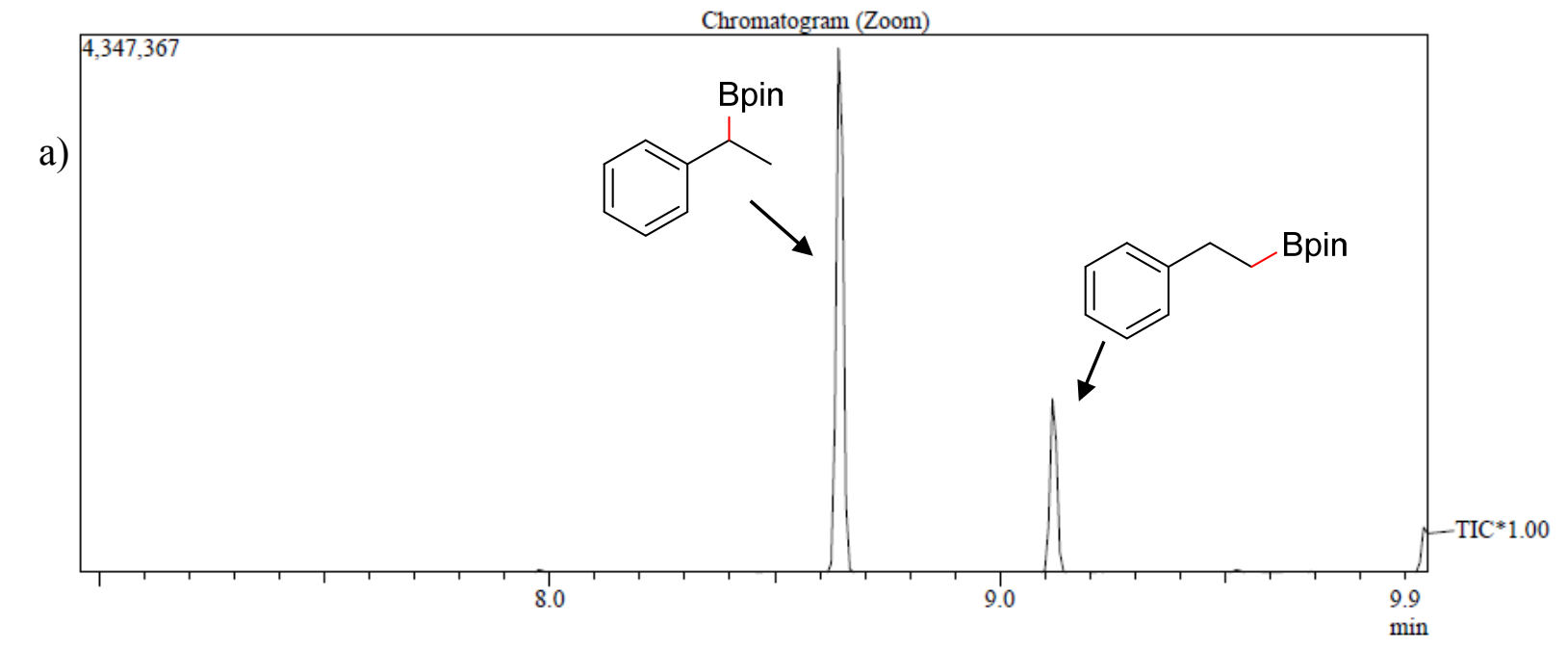

Line\#:1 R.Time:8.642(Scan\#:918)

MassPeaks: 438

RawMode:Single 8.642(918) BasePeak:105.10(413644)

BG Mode:None

b)

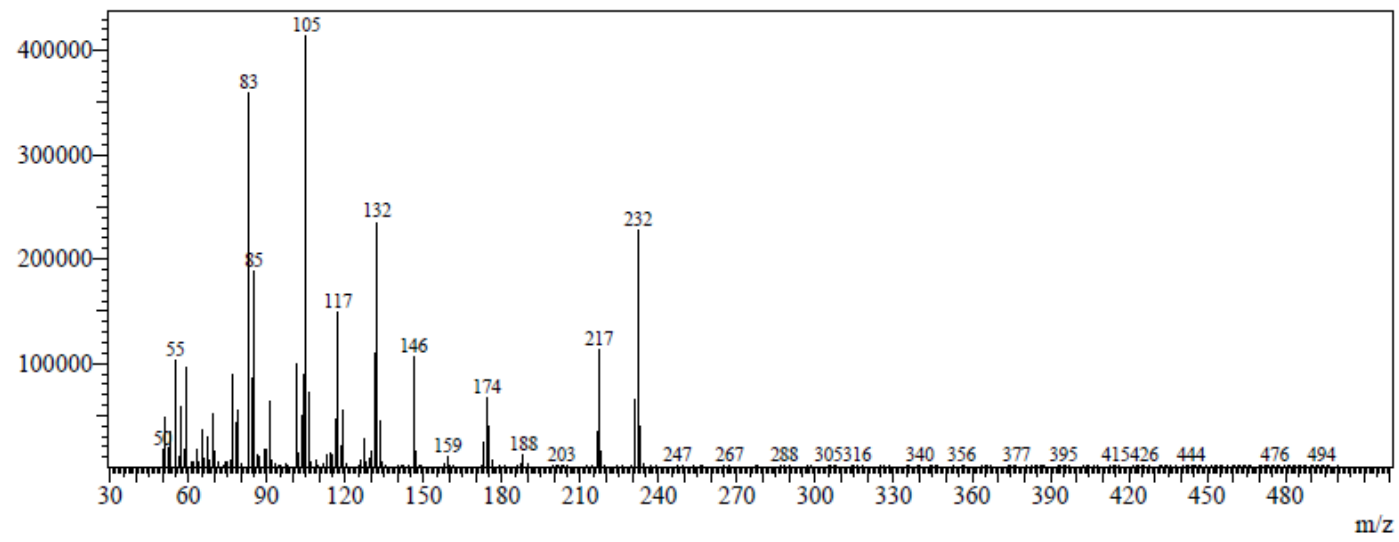

Figure S8. GC-MS chromatograms of authentic samples of the branched and linear borylated products. 

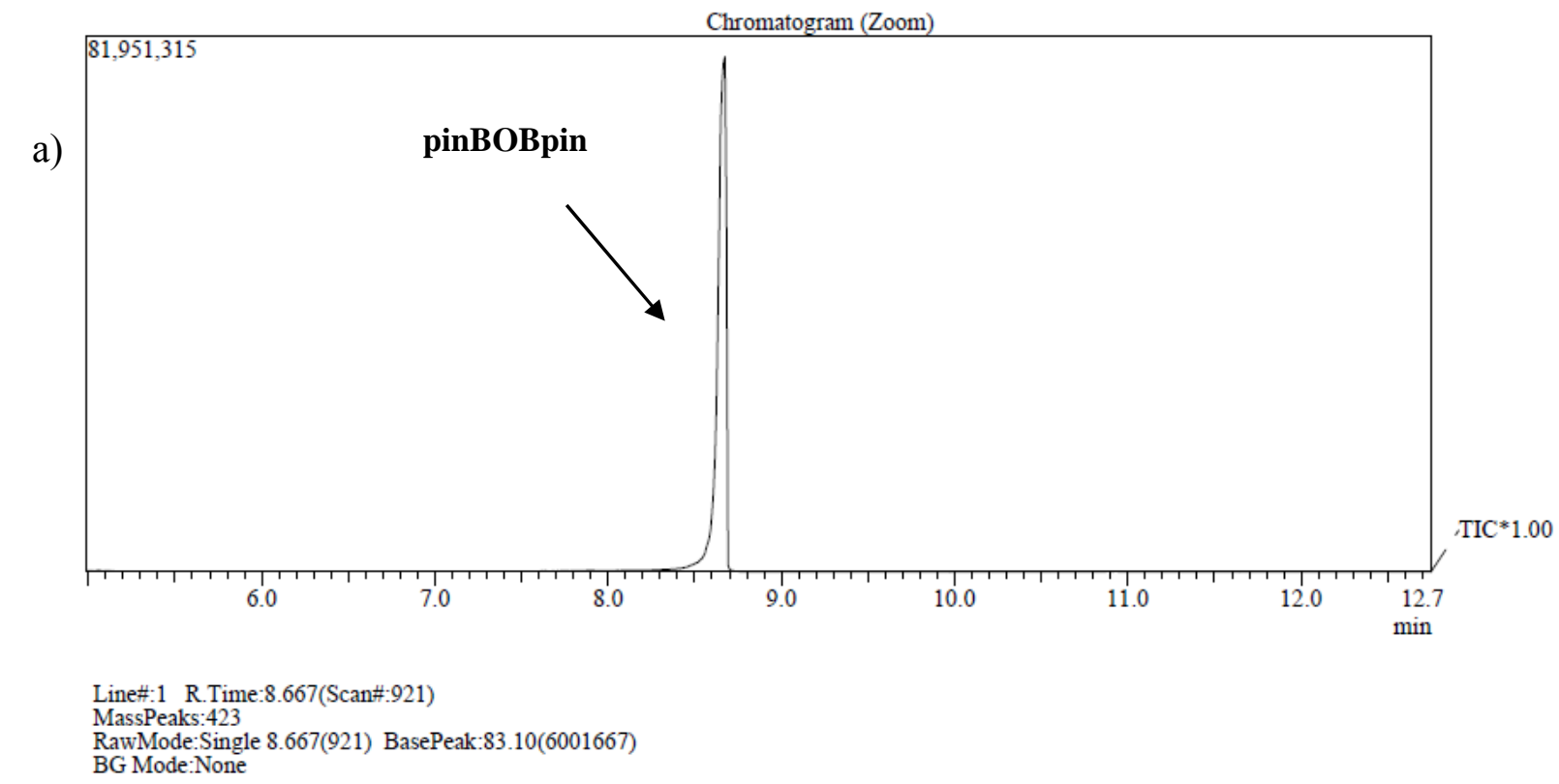
BG Mode:None

b)

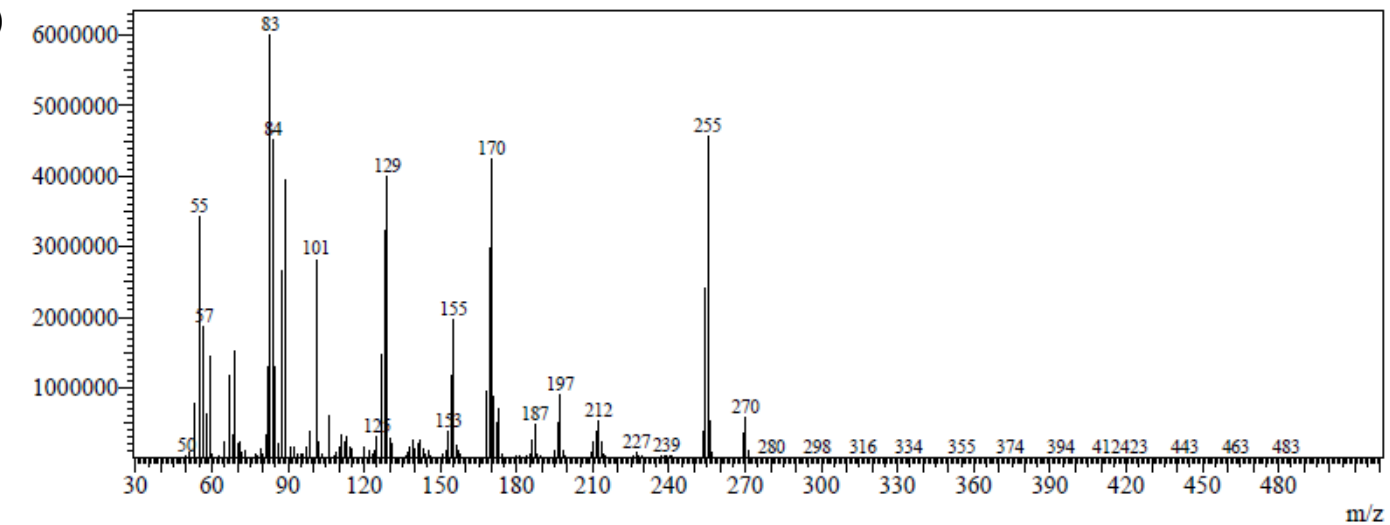

Figure S9. GC-MS chromatograms of authentic sample of 2,2'-oxybis(4,4,5,5-tetramethyl1,3,2,-dioxaborolane. The compound was independently synthesized according to Stephan's procedure see ref. $16 .{ }^{16}$ 
Chromam (Zoom)

a)

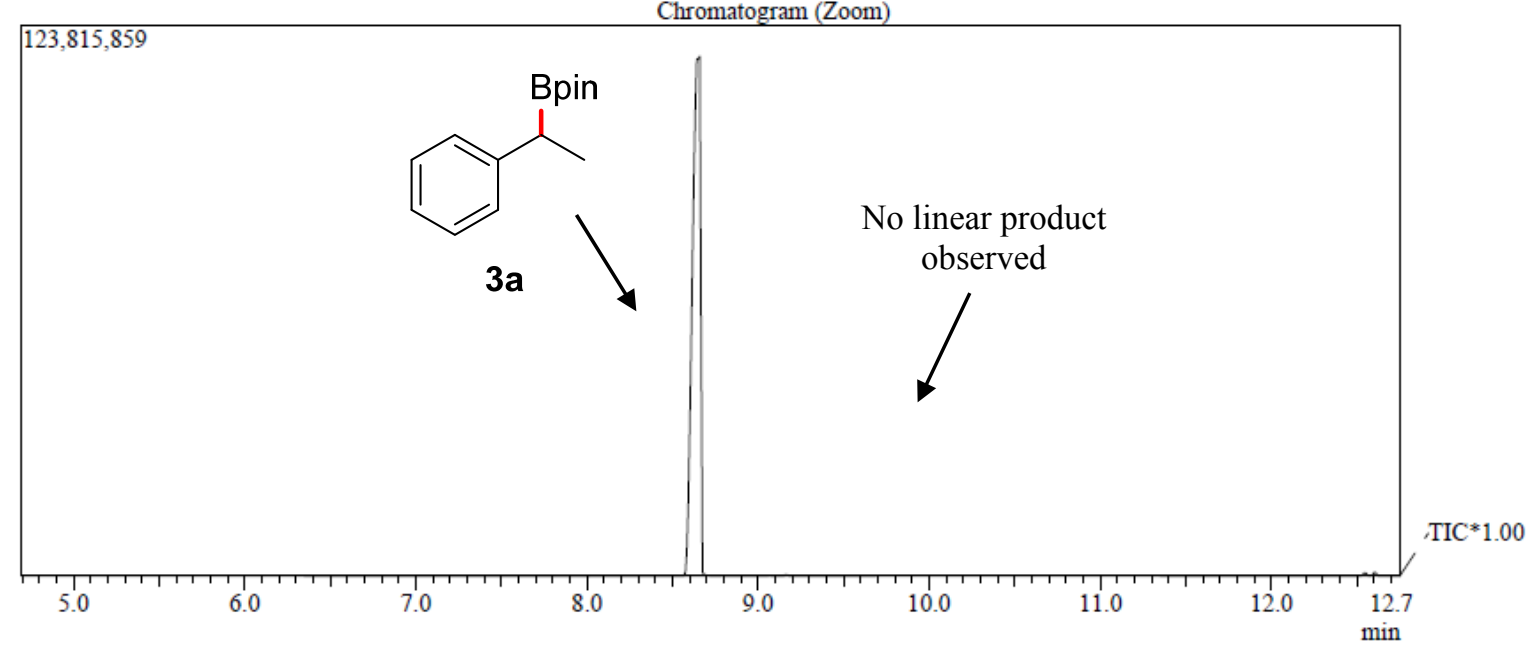

Line\#:1 R.Time:8.642(Scan\#:918)

MassPeaks: 430

RawMode:Single 8.642(918) BasePeak:104.95(14105095)

BG Mode:None

b)

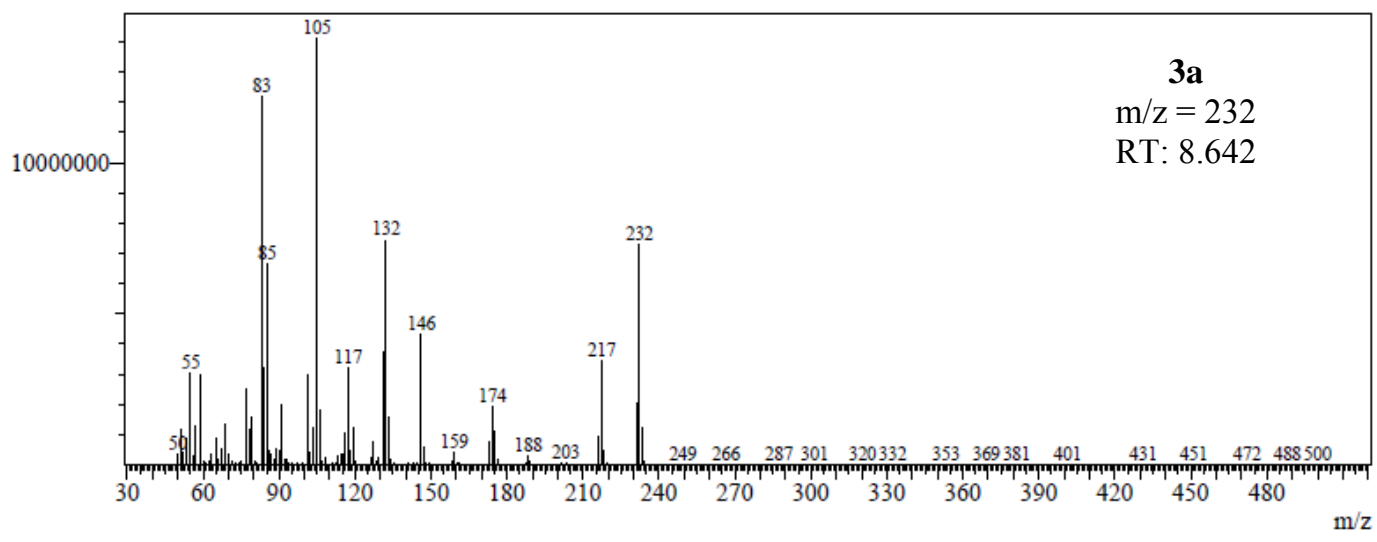

Figure S10. GC-MS chromatograms of product 3a. 
Chromatogram (Zoom)

a)

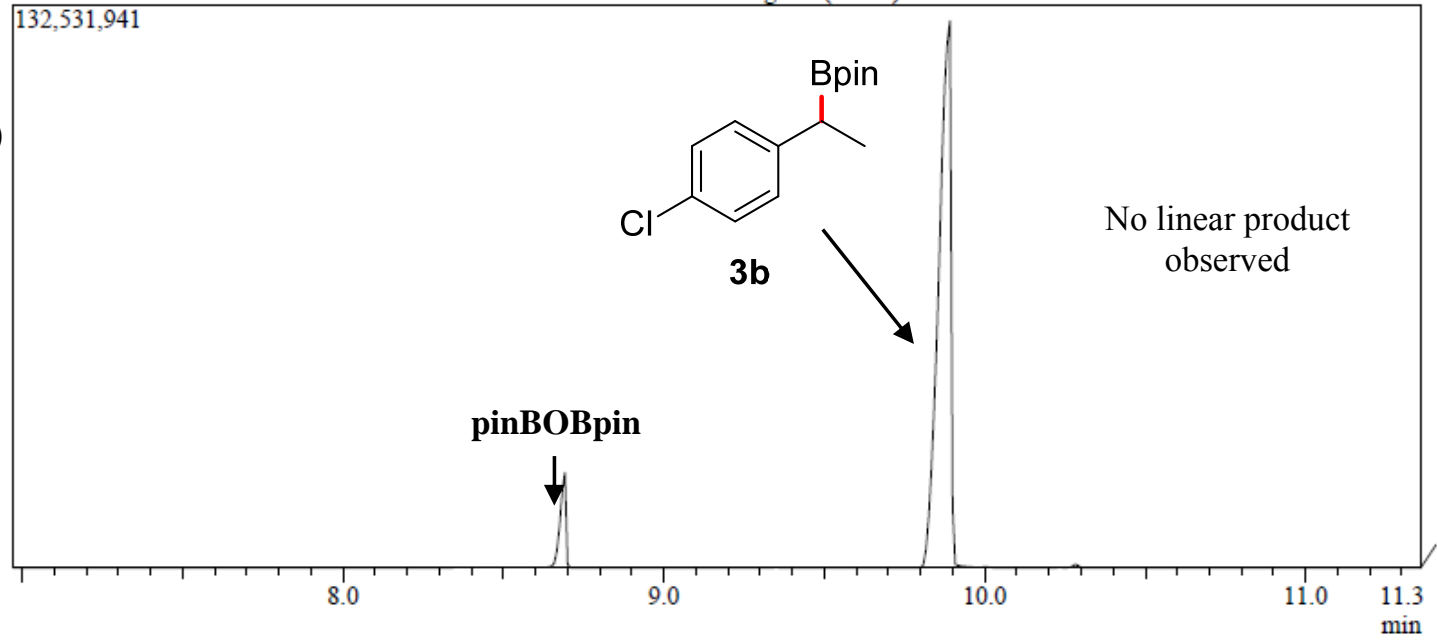

Line\#:1 R.Time:9.883(Scan\#:1067)

MassPeaks:434

RawMode:Single 9.883(1067) BasePeak:231.15(14238739)

BG Mode:None

b)

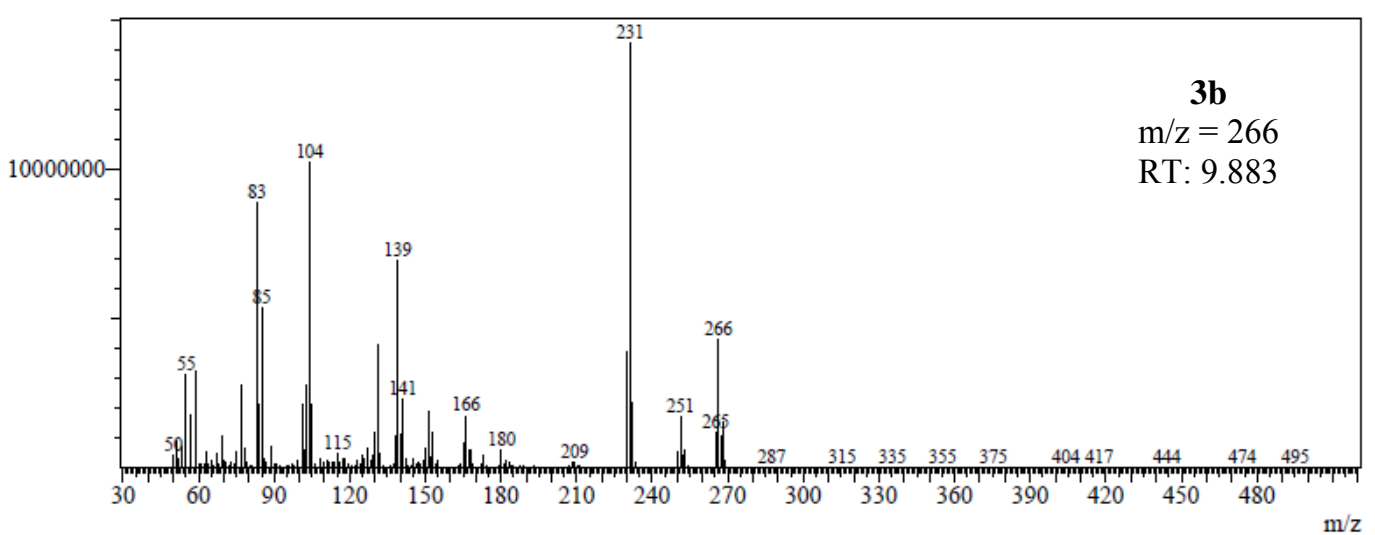

Line\#:1 R.Time:8.683(Scan\#:923)

MassPeaks: 437

RawMode:Single 8.683(923) BasePeak:255.20(1446225)

BG Mode:None

c)

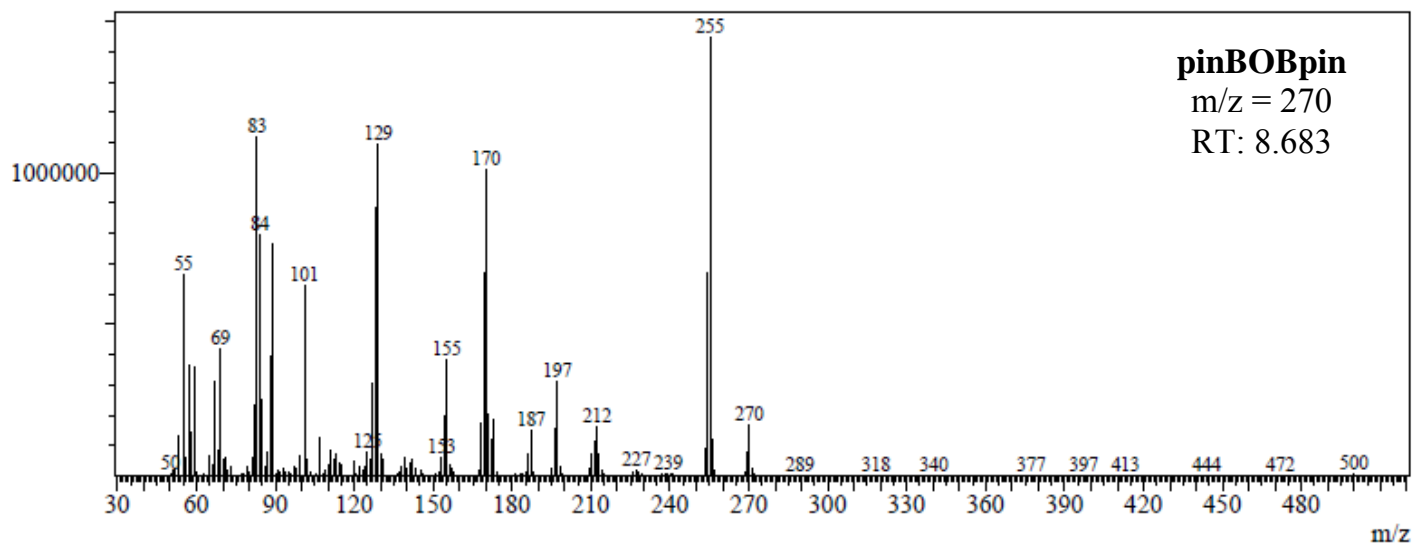

Figure S11. GC-MS chromatograms of product $\mathbf{3 b}$ and pinBOBpin. 
Chromatogram (Zoom)

a)

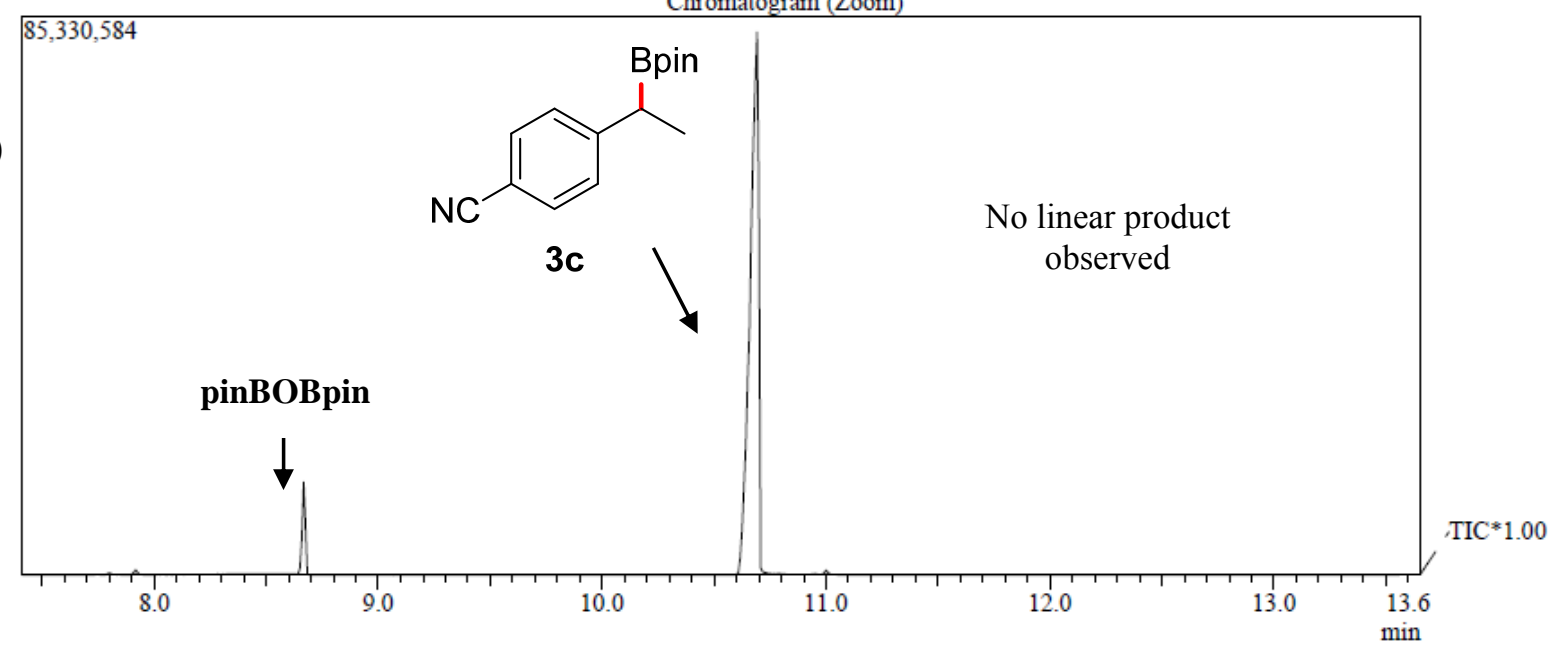

Line\#:1 R.Time:10.683(Scan\#:1163)

MassPeaks: 436

RawMode:Single 10.683(1163) BasePeak:83.10(8881072)

BG Mode:None

b)

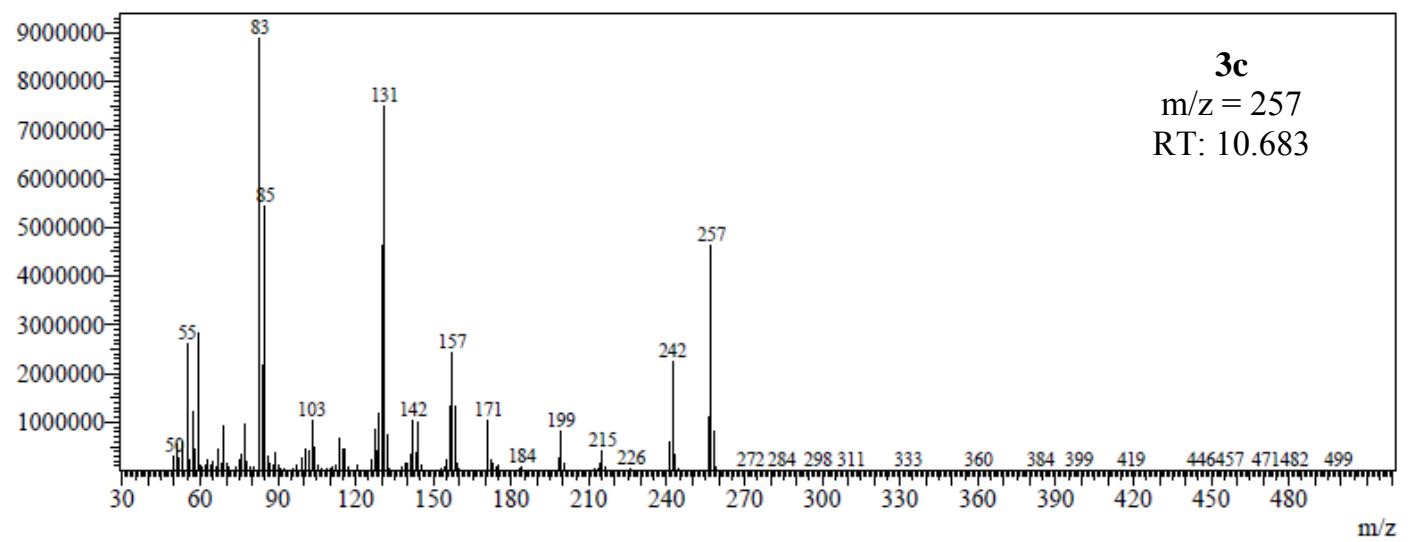

Line\#:1 R.Time:8.667(Scan\#:921)

MassPeaks:438

RawMode:Single 8.667(921) BasePeak:255.25(1074646)

BG Mode:None

c)

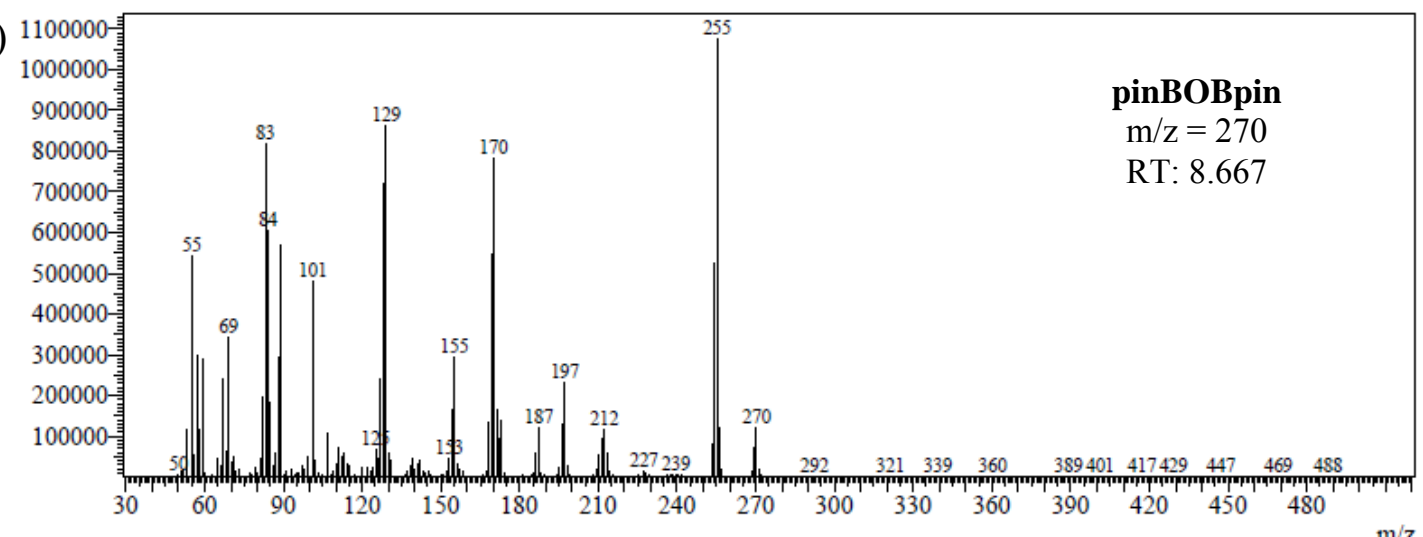

Figure S12. GC-MS chromatograms of product 3c and pinBOBpin. 
Chromatogram (Zoom)

a)

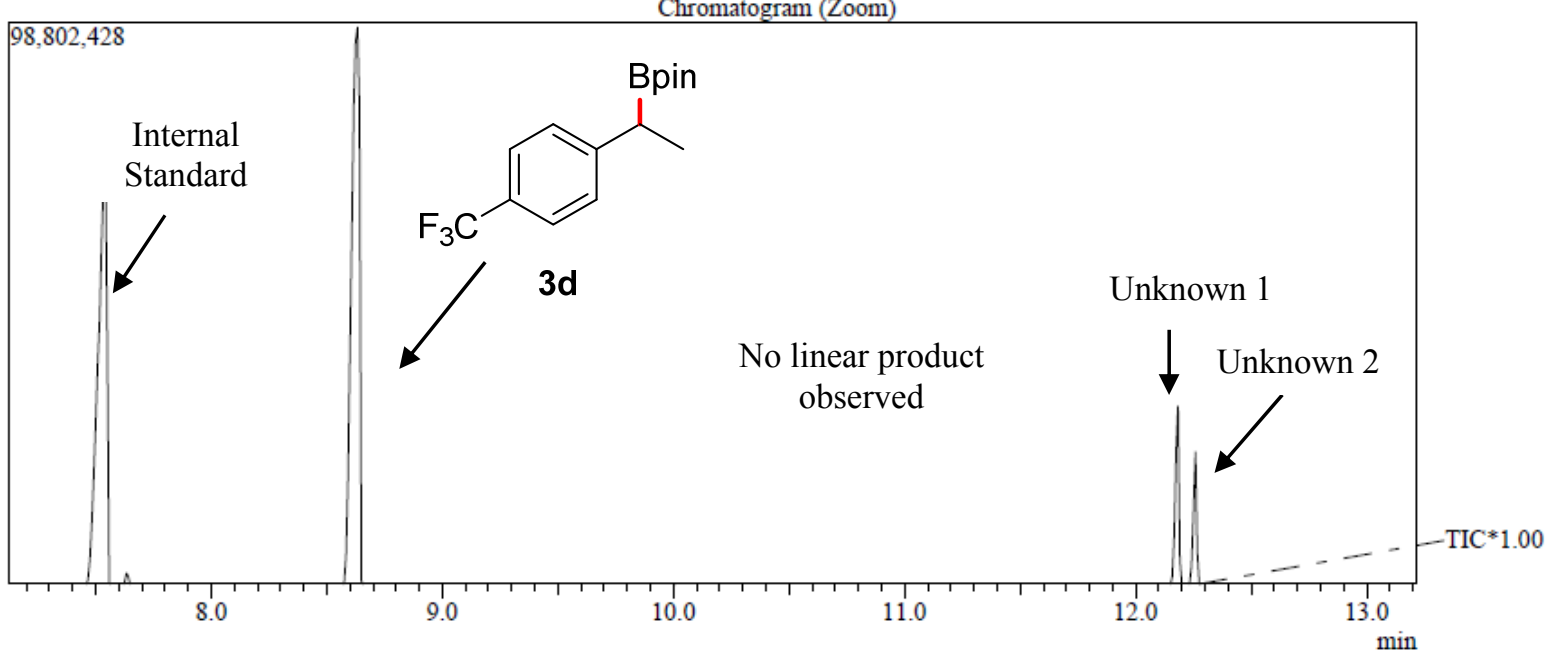

Line\#:1 R.Time:8.633(Scan\#:1036)

MassPeaks: 413

RawMode:Single 8.633(1036) BasePeak:153.95(13998804)

BG Mode:None

b)

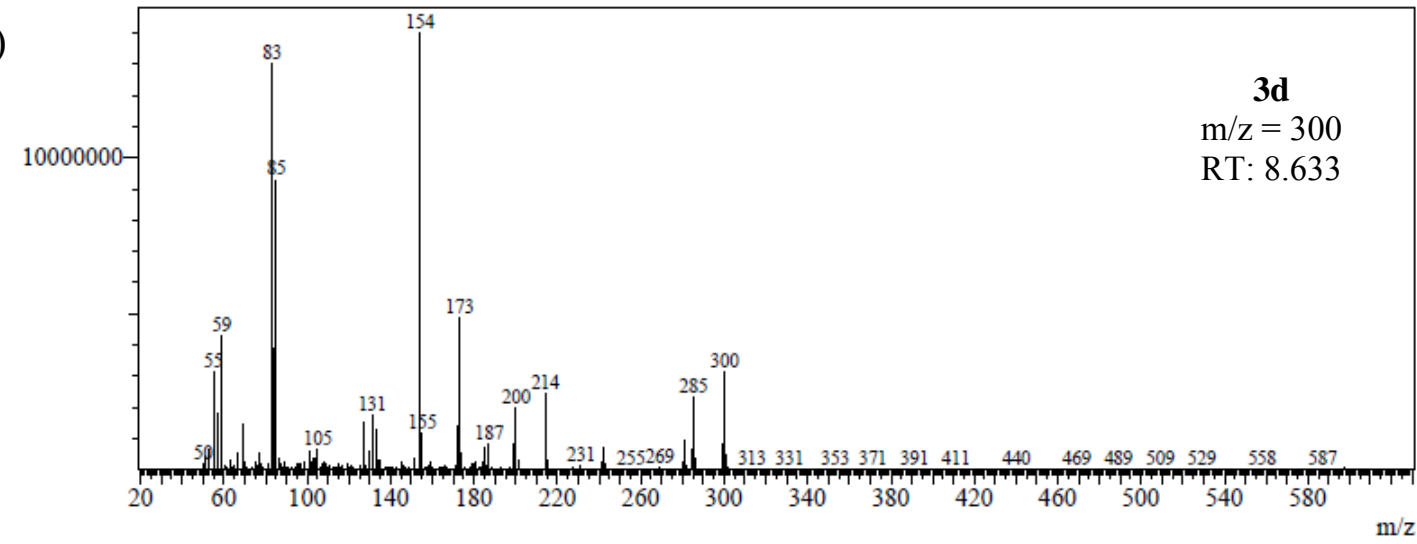

Line\#:1 R.Time:12.183(Scan\#:1462)

MassPeaks:448

RawMode:Single 12.183(1462) BasePeak:83.05(3770516)

BG Mode-None

c)

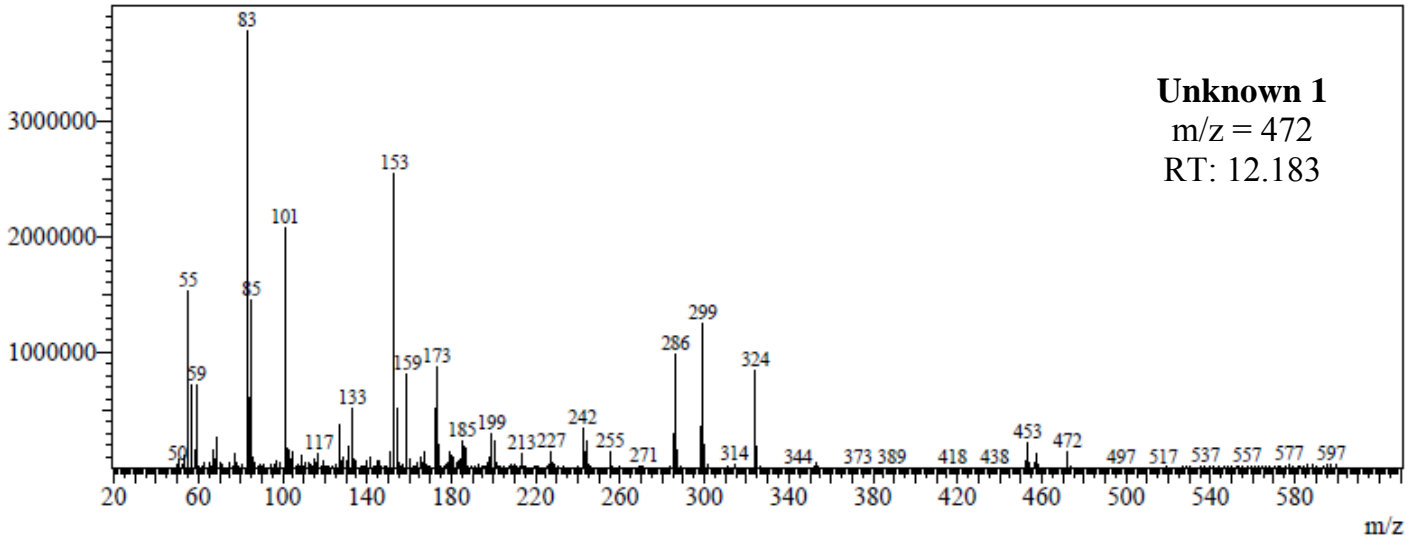


Line\#:1 R.Time:12.258(Scan\#:1471)

MassPeaks: 446

RawMode:Single 12.258(1471) BasePeak:83.05(2612904)

BG Mode:None

d)

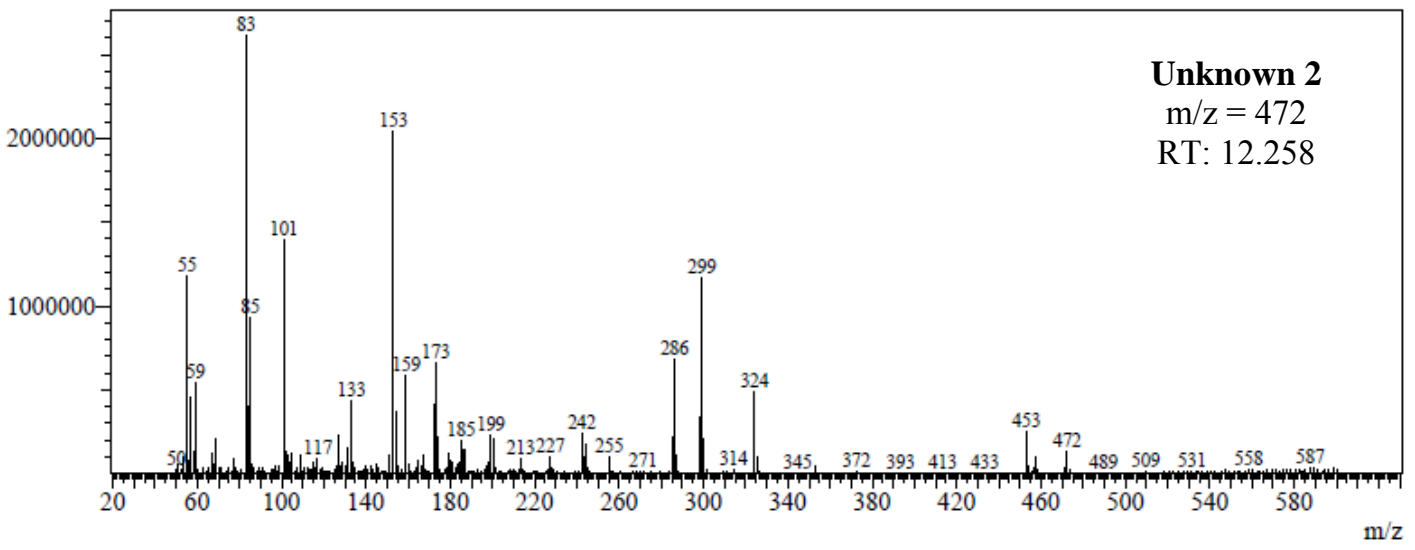

Figure S13. GC-MS chromatograms of product 3d and 2 unknowns. 
Chromatogram (Zoom)

a)
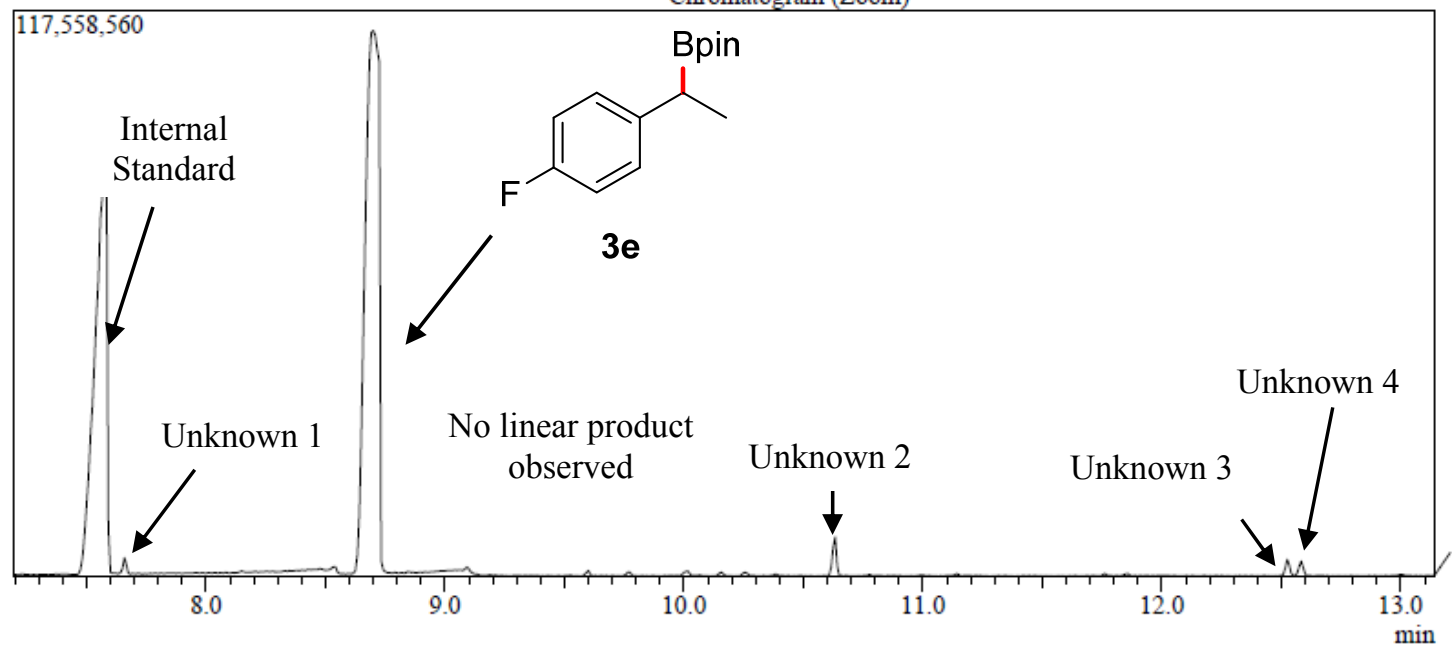

Line\#:1 R.Time:8.700(Scan\#:1044)

MassPeaks: 411

RawMode:Single 8.700(1044) BasePeak:83.05(12792463)

BG Mode:None

b)

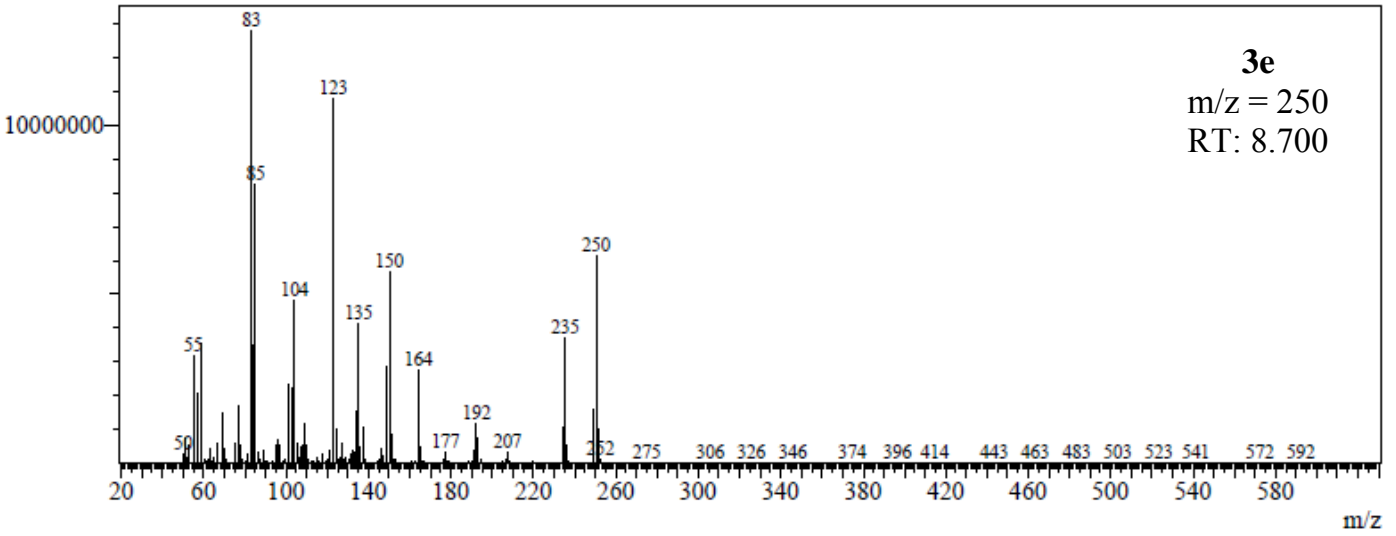

Line\#:1 R.Time:7.658(Scan\#:919)

MassPeaks:453

RawMode:Single 7.658(919) BasePeak:147.15(638476)

BG Mode:None

c)

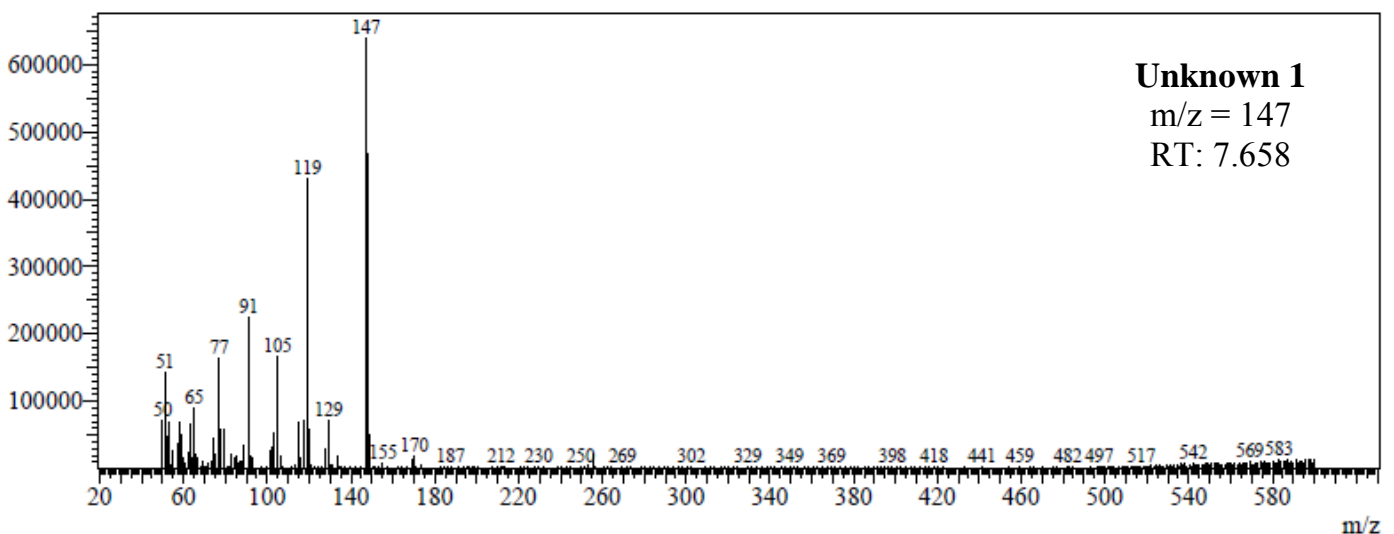


Line\#:1 R.Time:10.633(Scan\#:1276)

MassPeaks: 410

RawMode:Single 10.633(1276) BasePeak:133.10(1212694)

d)

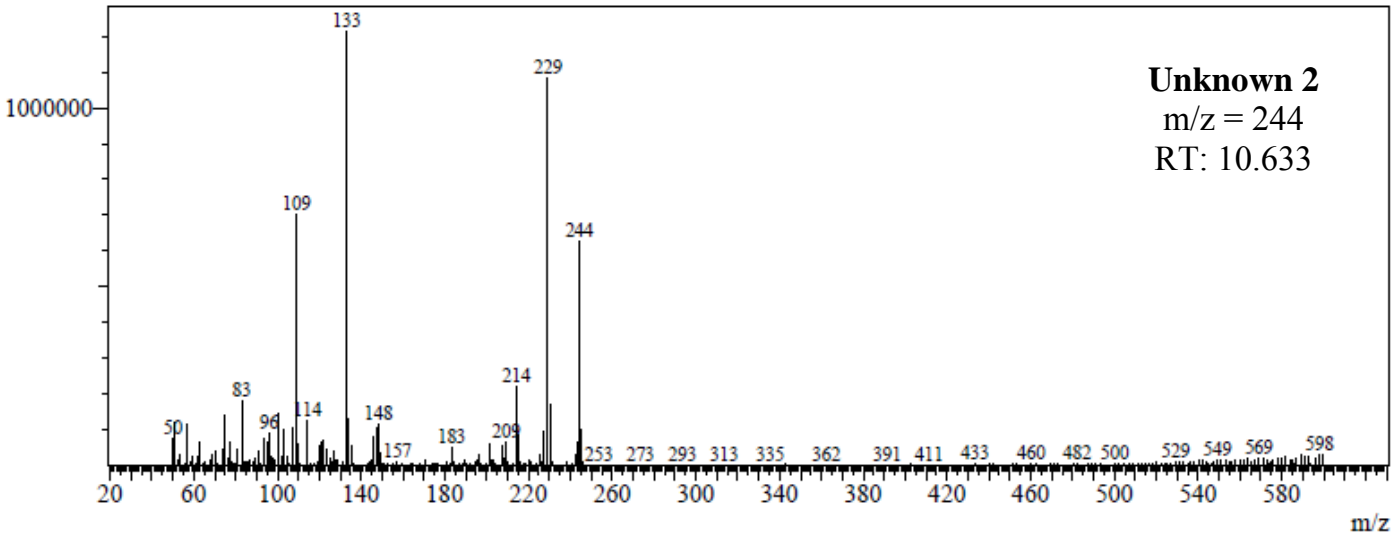

Line\#:1 R.Time:12.525(Scan\#:1503)

MassPeaks: 408

RawMode:Single 12.525(1503) BasePeak:83.10(288981)

BG Mode:None

e)

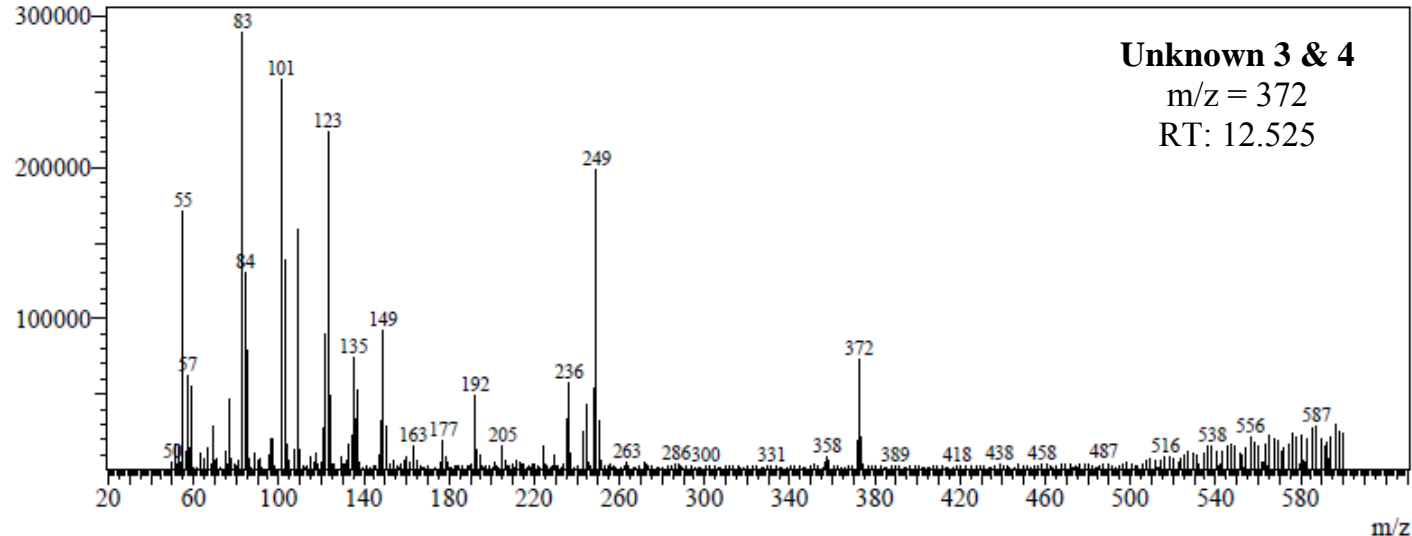

Figure S14. GC-MS chromatograms of product 3e and several unknowns. 
Chromatogram (Zoom)

a)

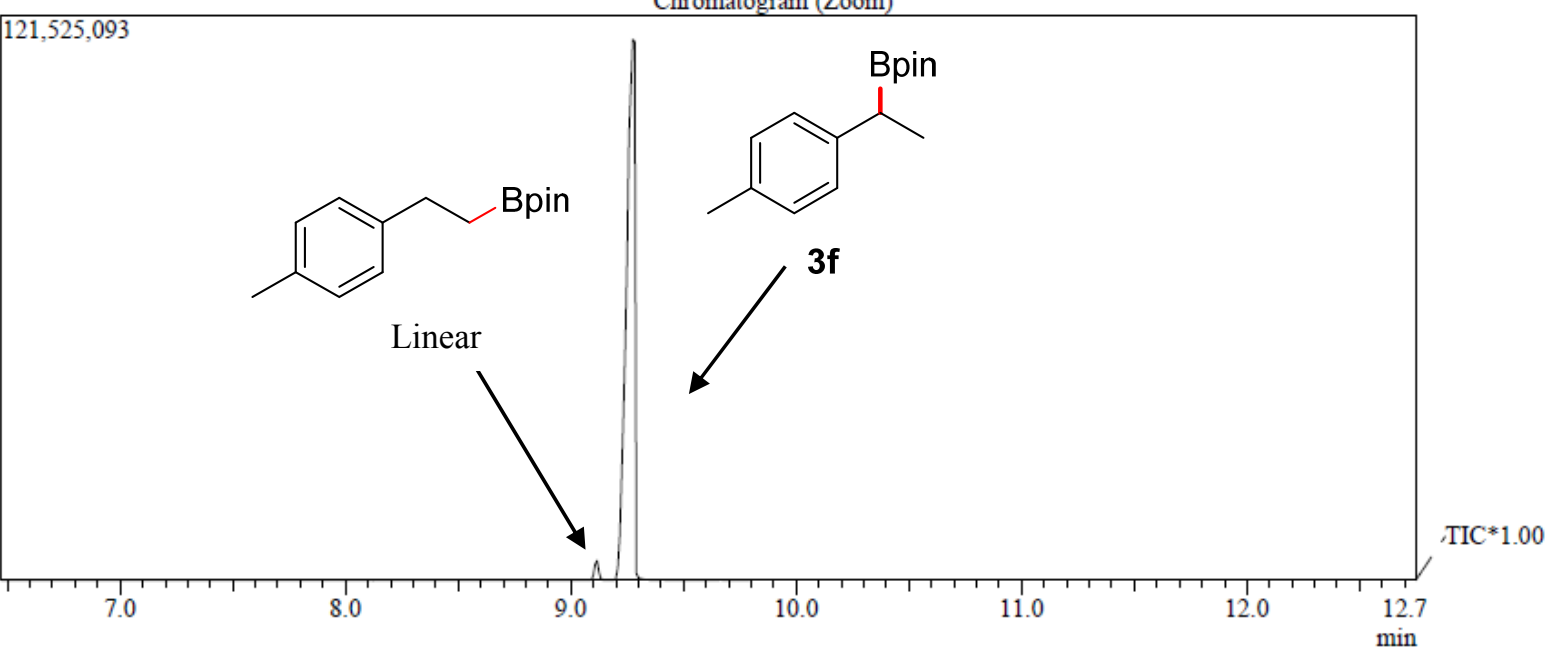

Line\#:1 R.Time:9.117(Scan\#:975)

MassPeaks:435

RawMode:Single 9.117(975) BasePeak:119.00(582652)

BG Mode:None

b)

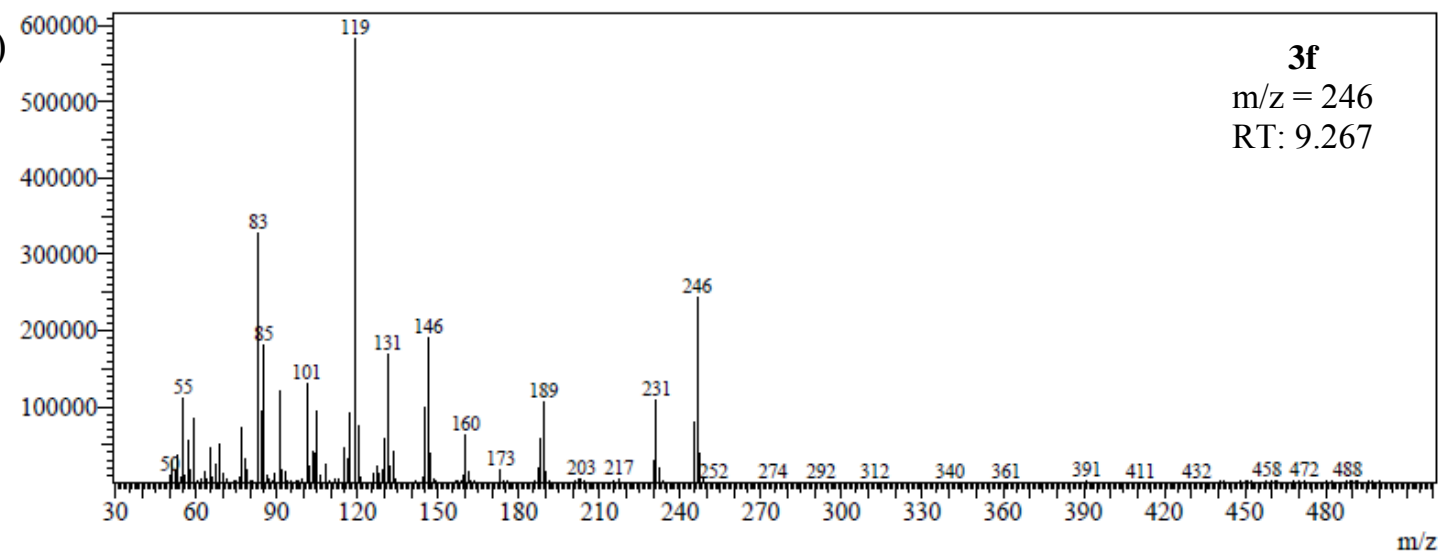

Line\#:1 R.Time:9.267(Scan\#:993)

MassPeaks: 426

RawMode:Single 9.267(993) BasePeak:119.15(11447471)

BG Mode:None

c)

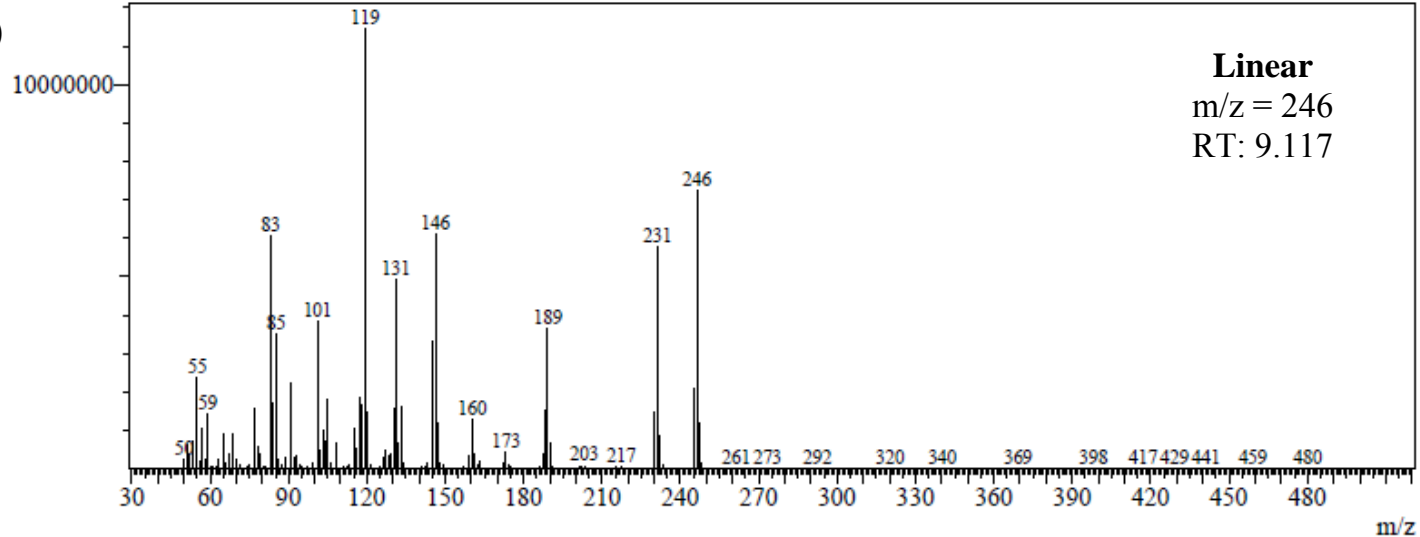

Figure S15. GC-MS chromatograms of product $\mathbf{3 f}$ and linear isomer. 
a)

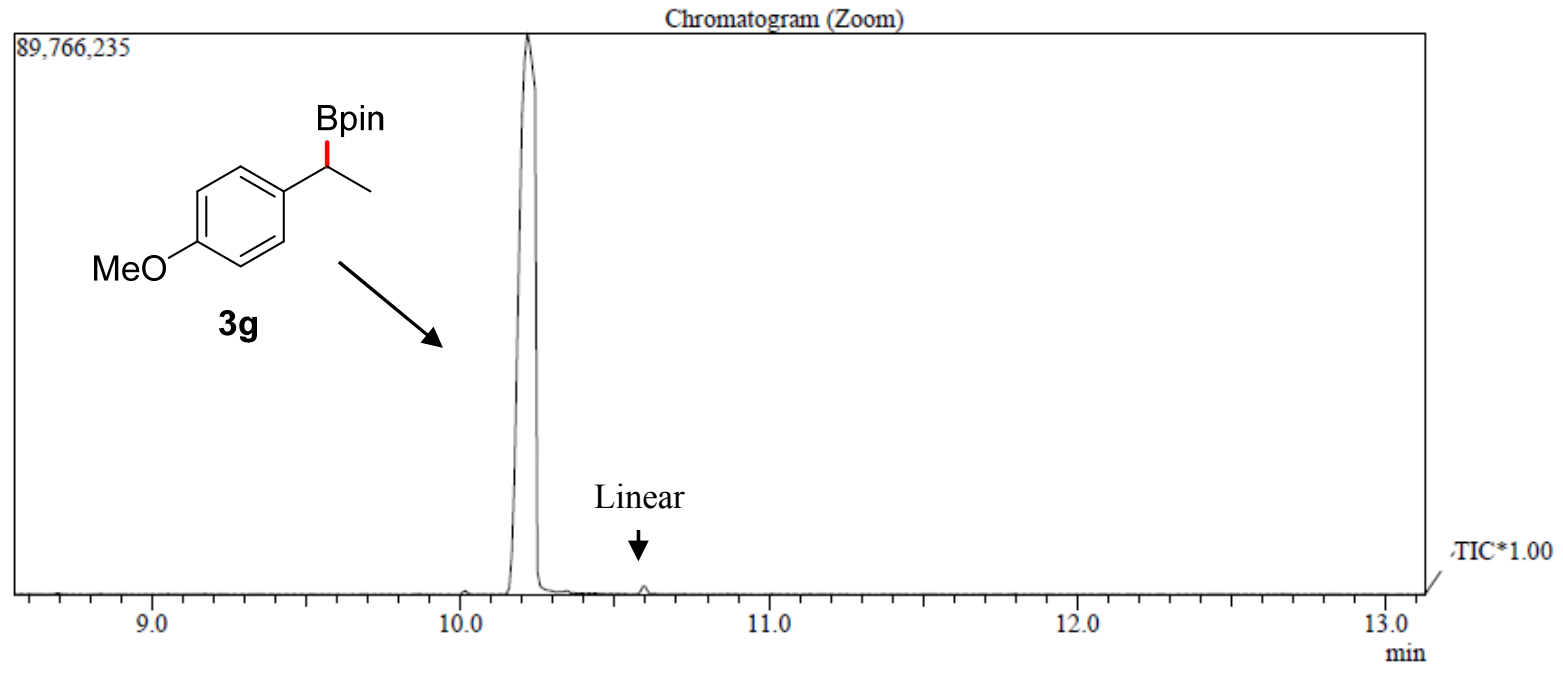

Line\#:1 R.Time:10.208(Scan\#:1225)

MassPeaks:441

RawMode:Single 10.208(1225) BasePeak:247.25(15673430)

BG Mode: None

b)

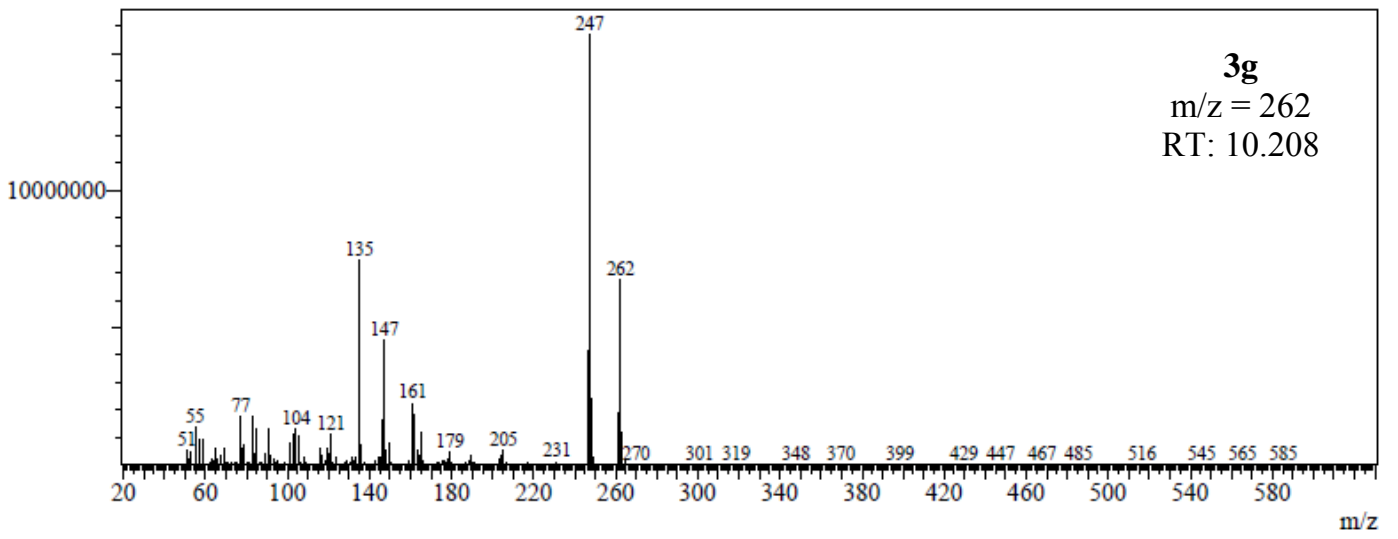

Line\#:1 R.Time:10.600(Scan\#:1272)

MassPeaks:418

RawMode:Single 10.600(1272) BasePeak:121.10(269142)

BG Mode:None

c)

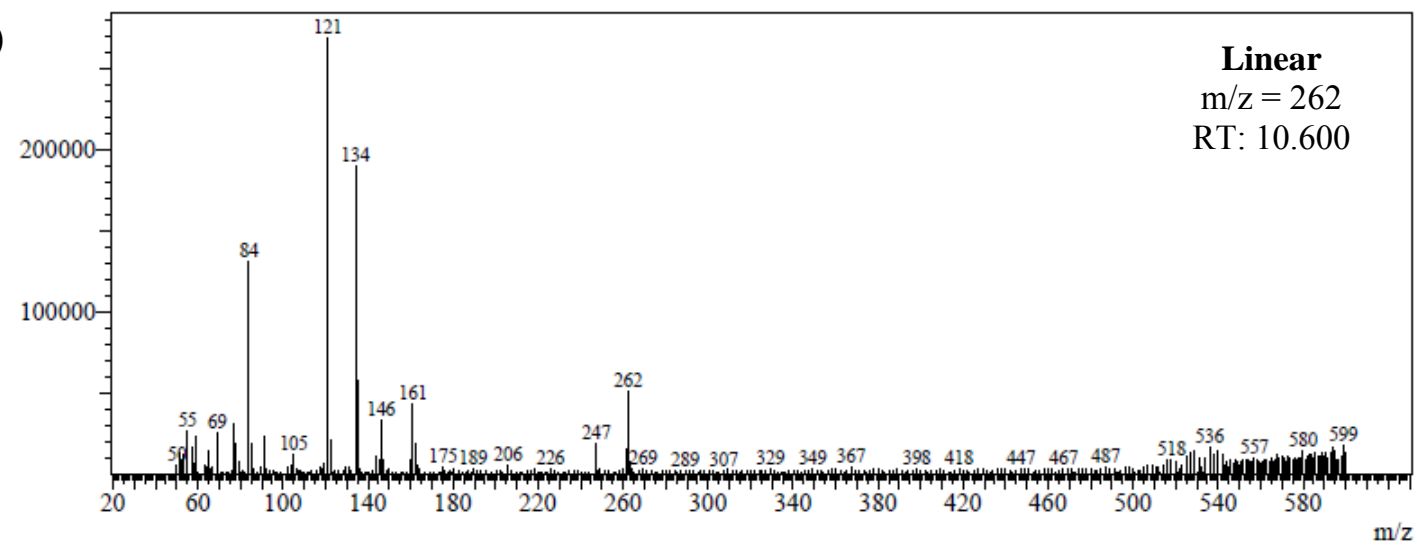

Figure S16. GC-MS chromatograms of product $\mathbf{3 g}$ and linear isomer. 
a)

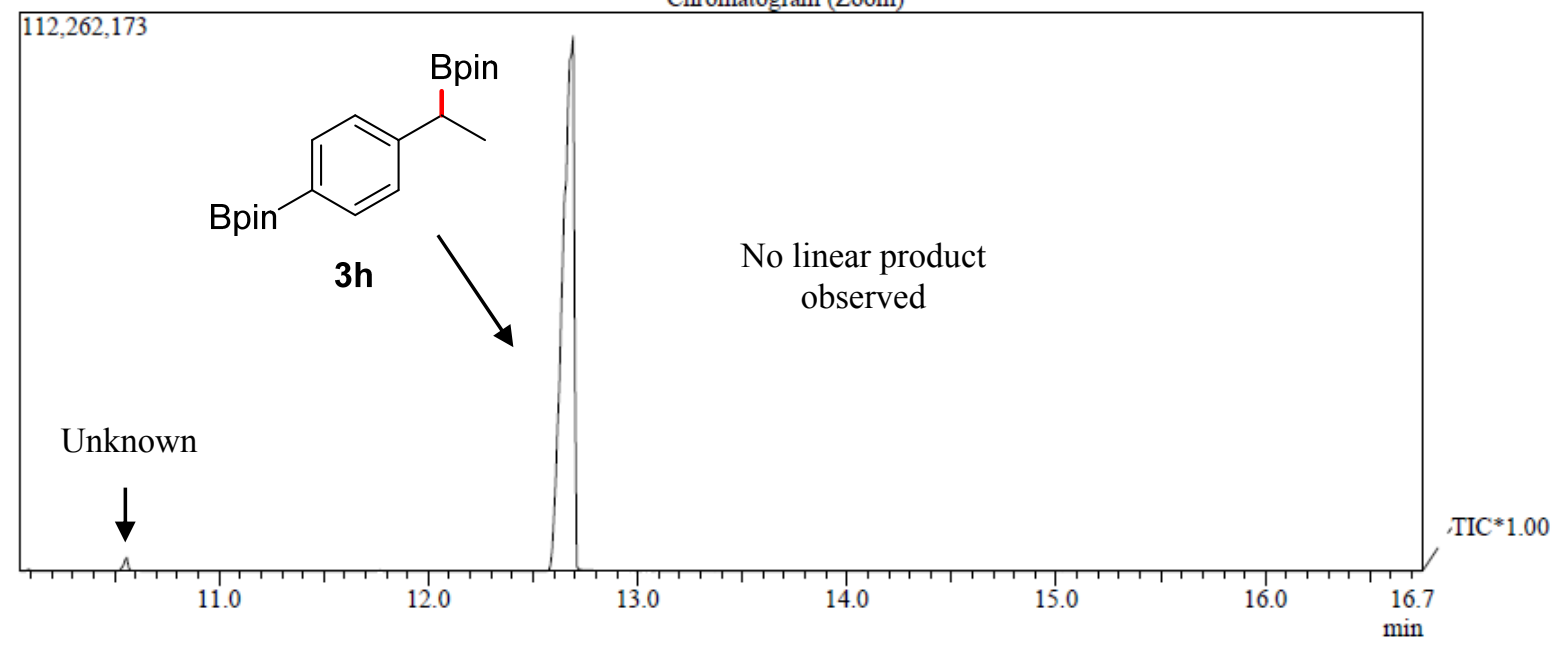

Line\#:1 R.Time:12.667(Scan\#:1401)

MassPeaks: 420

RawMode:Single 12.667(1401) BasePeak:83.05(16606880)

BG Mode:None

b)

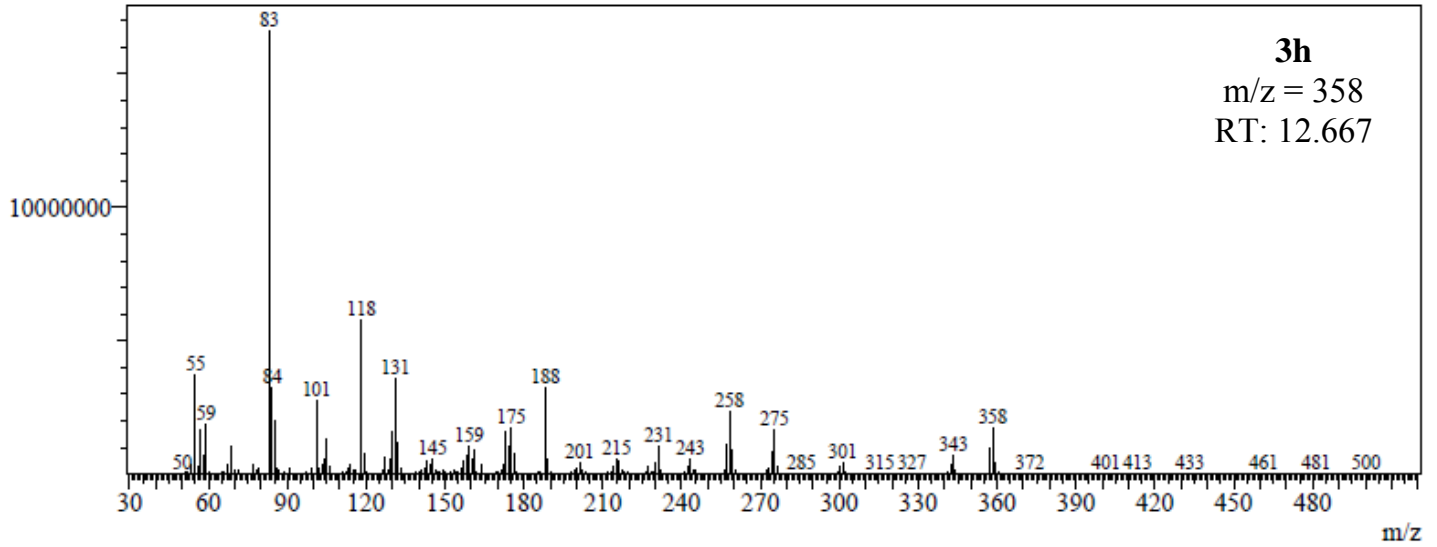

Line\#:1 R.Time:10.550(Scan\#:1147)

MassPeaks:431

RawMode:Single 10.550(1147) BasePeak:231.15(380656)

BG Mode:None

c)

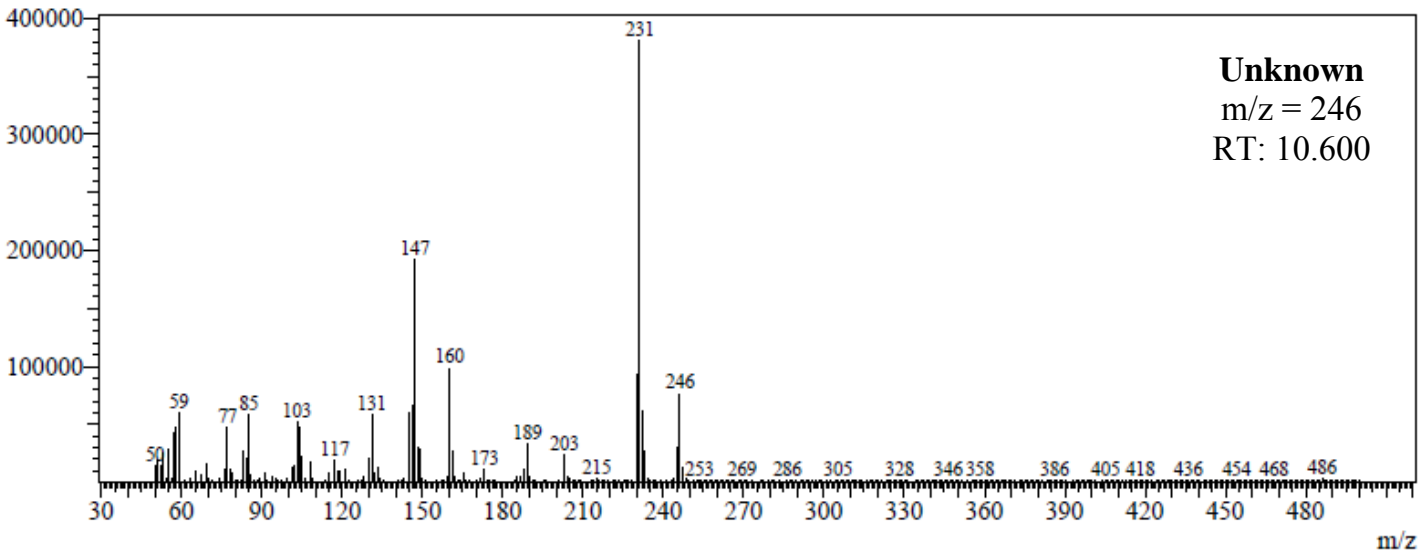

Figure S17. GC-MS chromatograms of product $\mathbf{3 h}$ and an unknown. 
Chromatogram (Zoom)

a)

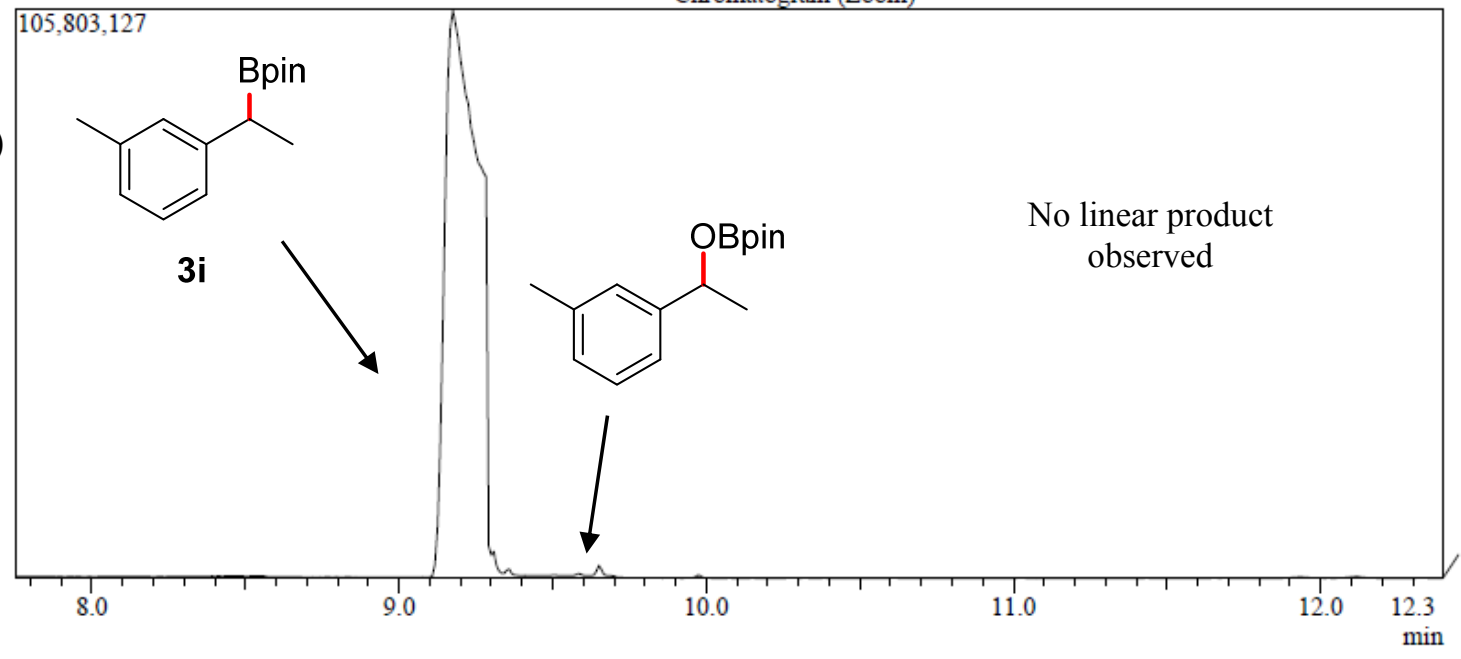

Line\#:1 R.Time:9.217(Scan\#:1106)

MassPeaks: 423

RawMode:Single 9.217(1106) BasePeak:118.95(10515452)

BG Mode:None

b)

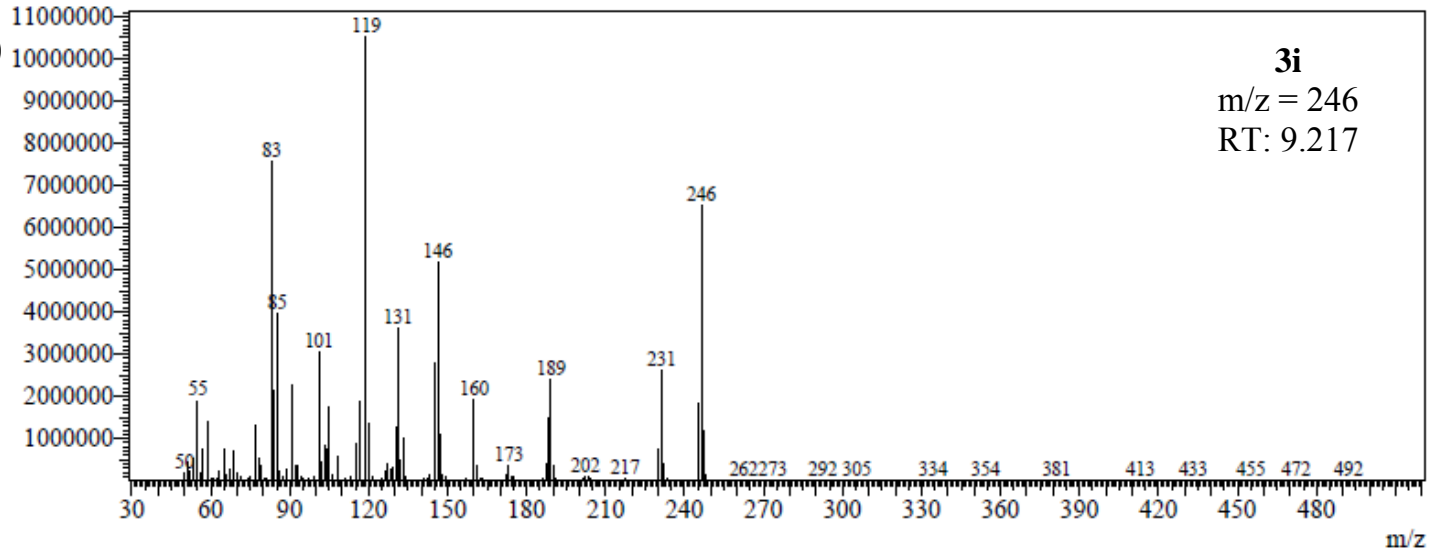

Line\#:1 R.Time:9.658(Scan\#:1159)

MassPeaks: 430

RawMode:Single 9.658(1159) BasePeak:84.00(316170)

BG Mode: None

c)

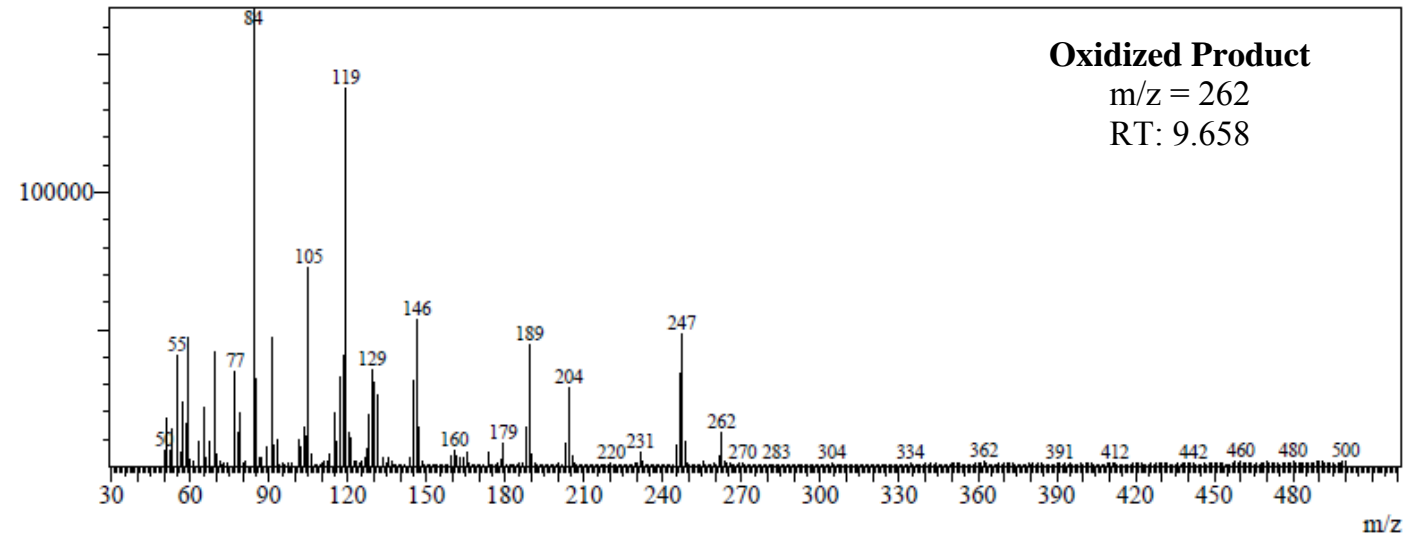

Figure S18. GC-MS chromatograms of product $3 \mathbf{i}$ and oxidized product. Assignment of oxidized product is tentative. 
a)

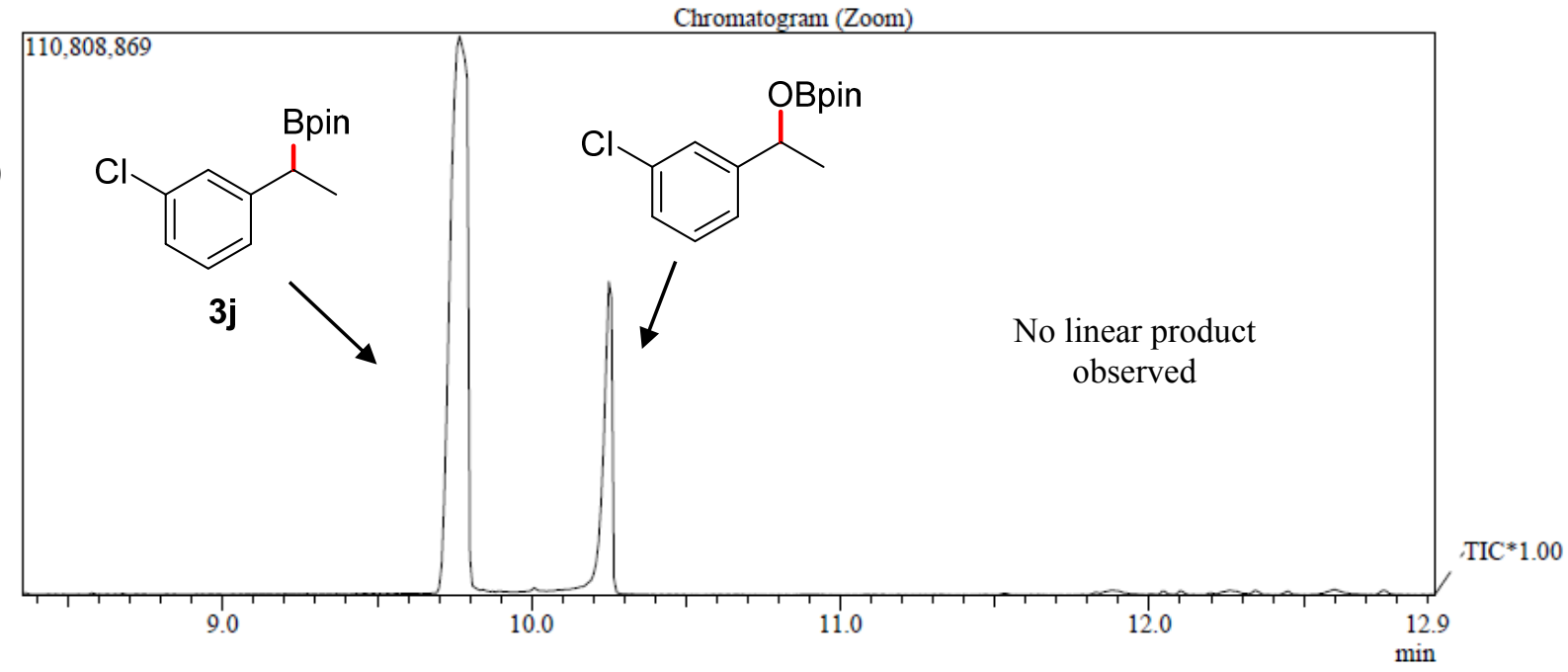

Line\#:1 R.Time:9.758(Scan\#:1171)

MassPeaks: 417

RawMode:Single 9.758(1171) BasePeak:83.05(13964098)

BG Mode:None

b)

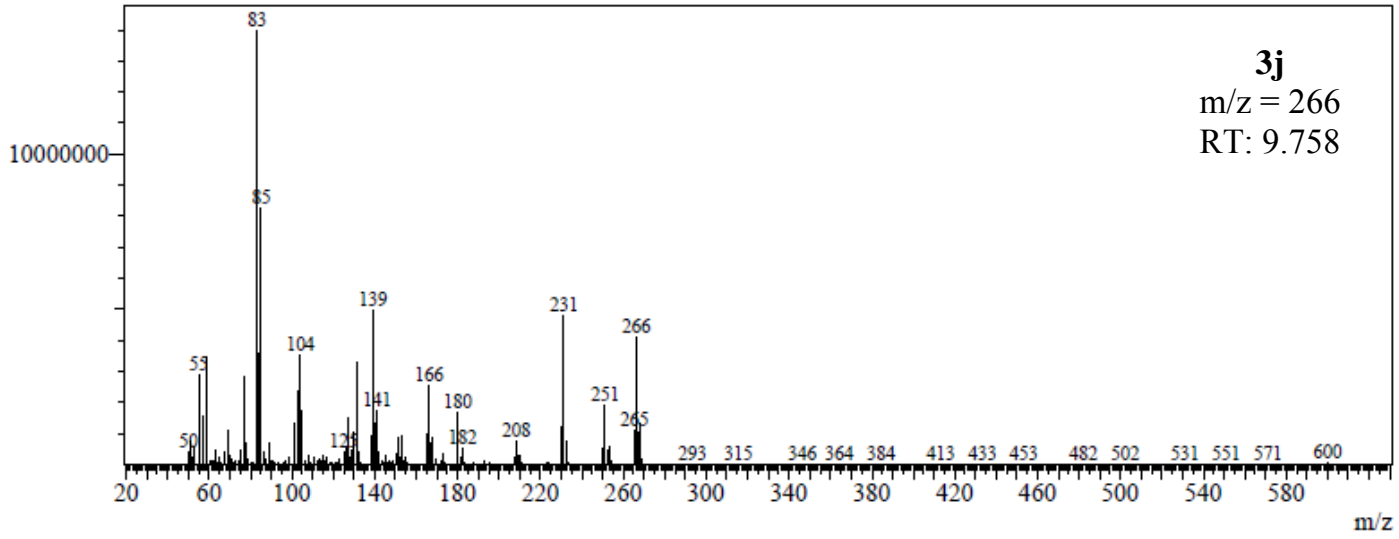

Line\#:1 R.Time:10.250(Scan\#:1230)

MassPeaks:421

RawMode:Single 10.250(1230) BasePeak:138.95(6382807)

BG Mode:None

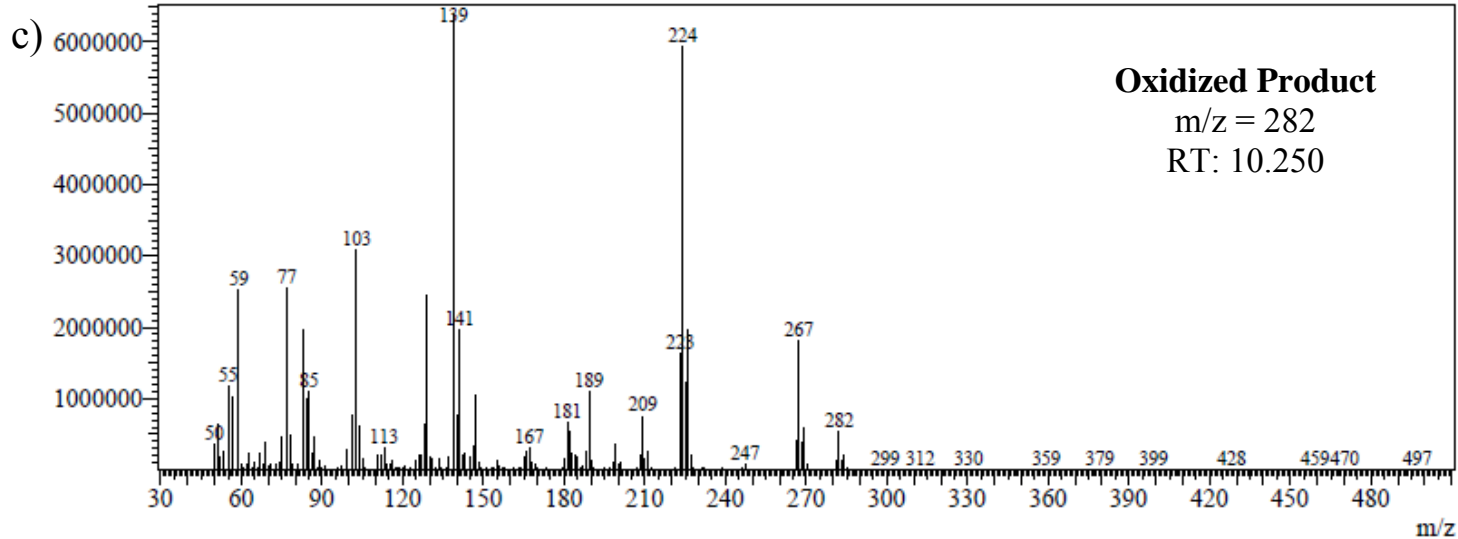

Figure S19. GC-MS chromatogram of product 3j and oxidized product. Assignment of oxidized product is tentative. 
Chromatogram (Zoom)

a)

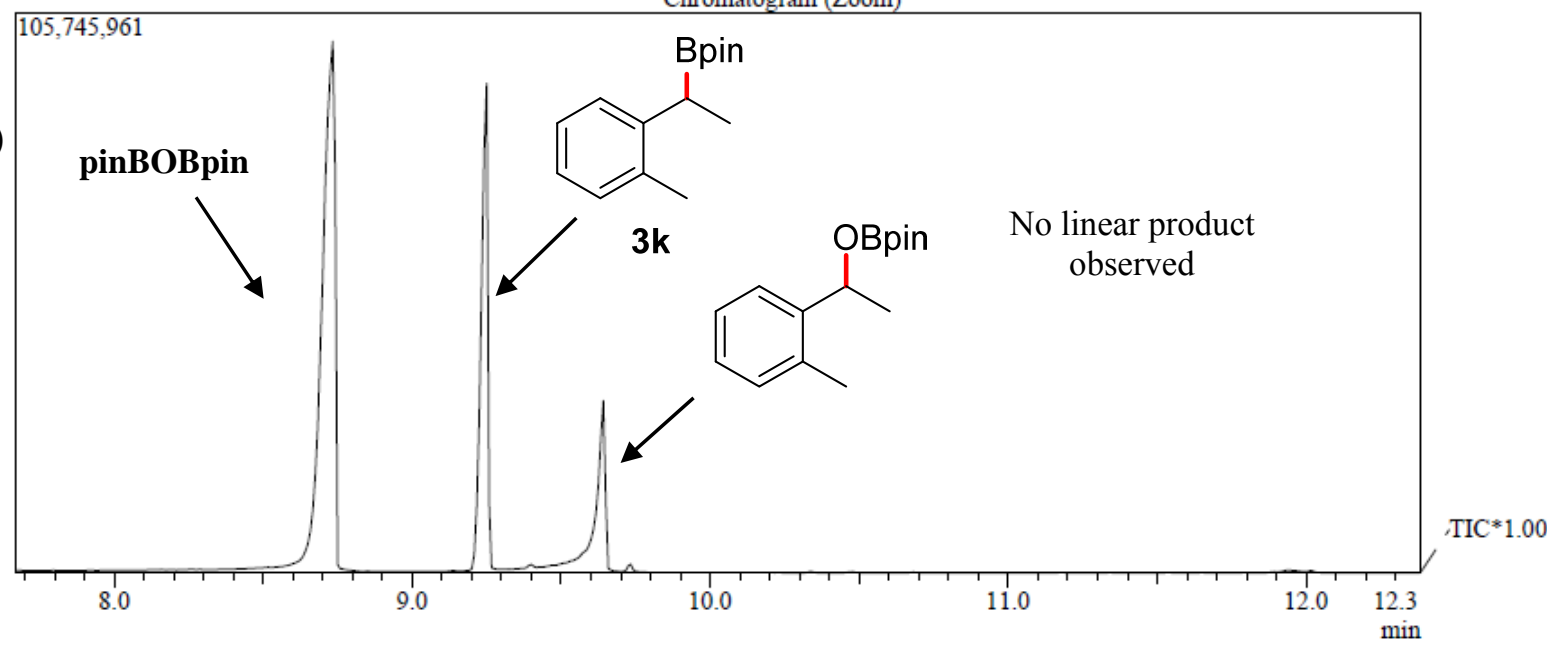

Line\#:1 R.Time:9.242(Scan\#:1109)

MassPeaks:434

RawMode:Single 9.242(1109) BasePeak:189.20(5942153)

BG Mode:None

b)

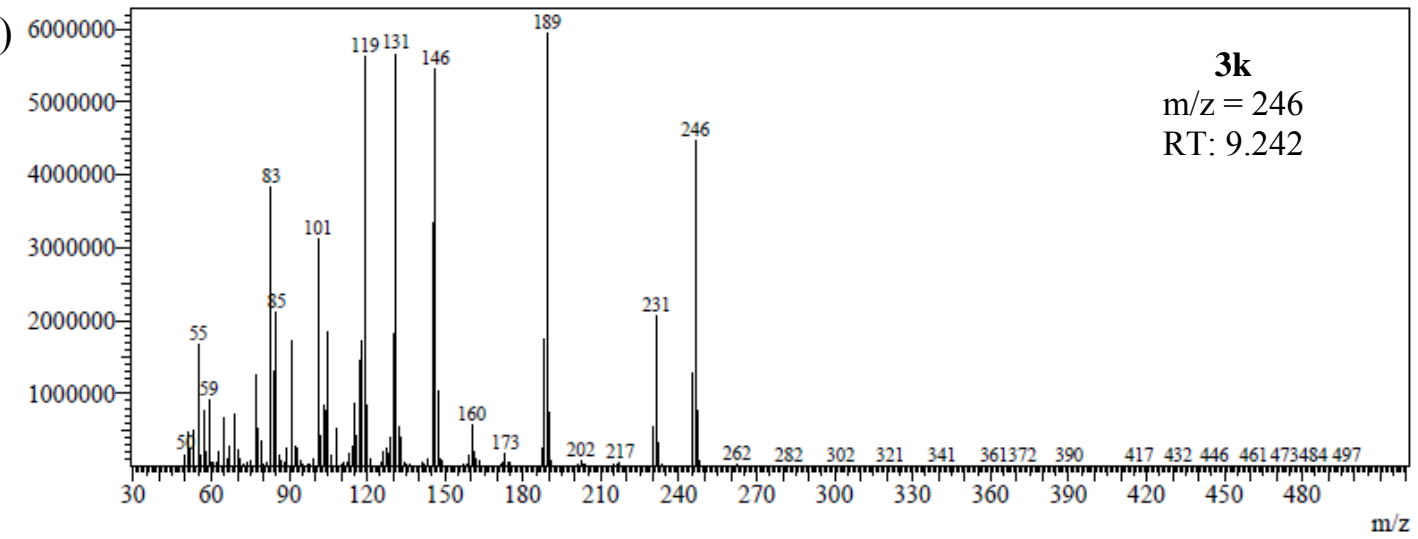

Line\#:1 R.Time:8.725(Scan\#:1047)

MassPeaks: 427

RawMode:Single 8.725(1047) BasePeak:255.20(6560777)

BG Mode:None

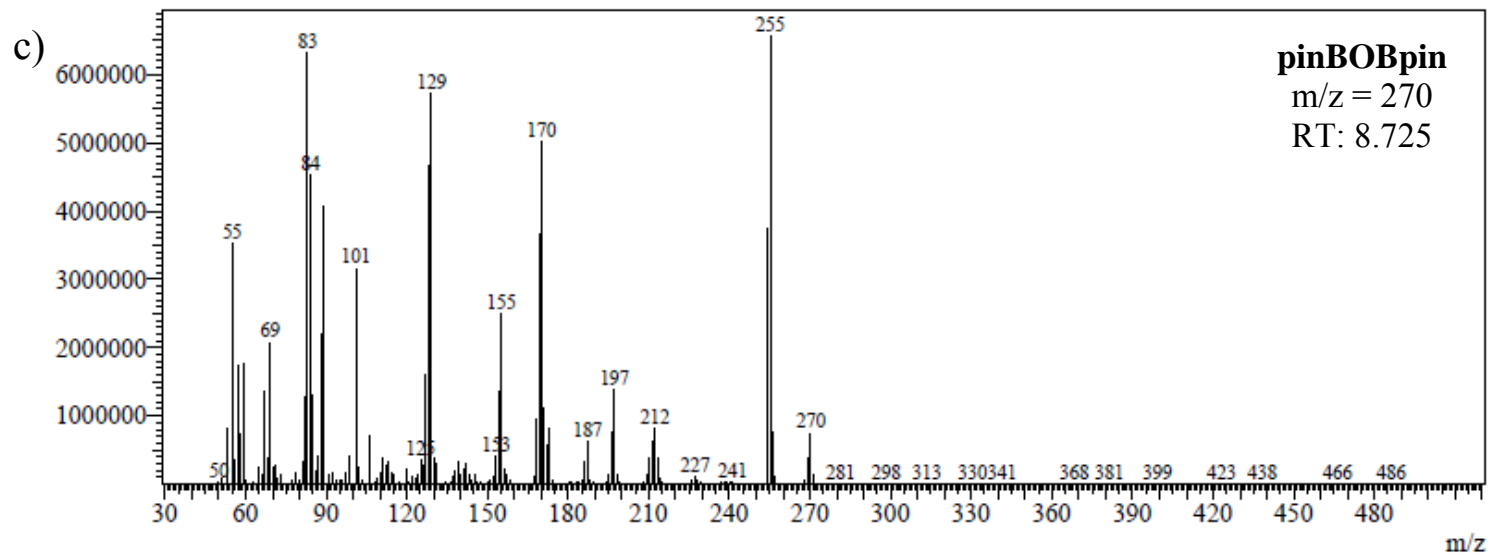


Line\#:1 R.Time:9.633(Scan\#:1156)

MassPeaks:429

RawMode:Single 9.633(1156) BasePeak:118.15(4049065)

BG Mode:None

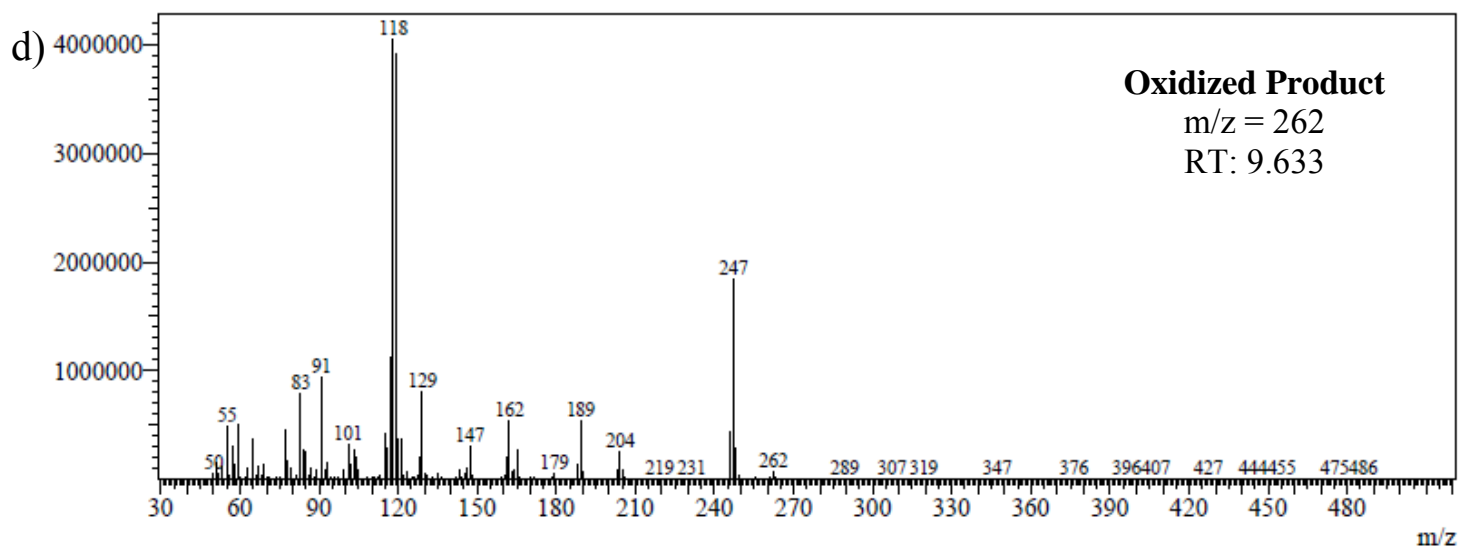

Figure S20. GC-MS chromatogram of product 3k, pinBOBpin, and oxidized product. Assignment of oxidized product is tentative. 
a)

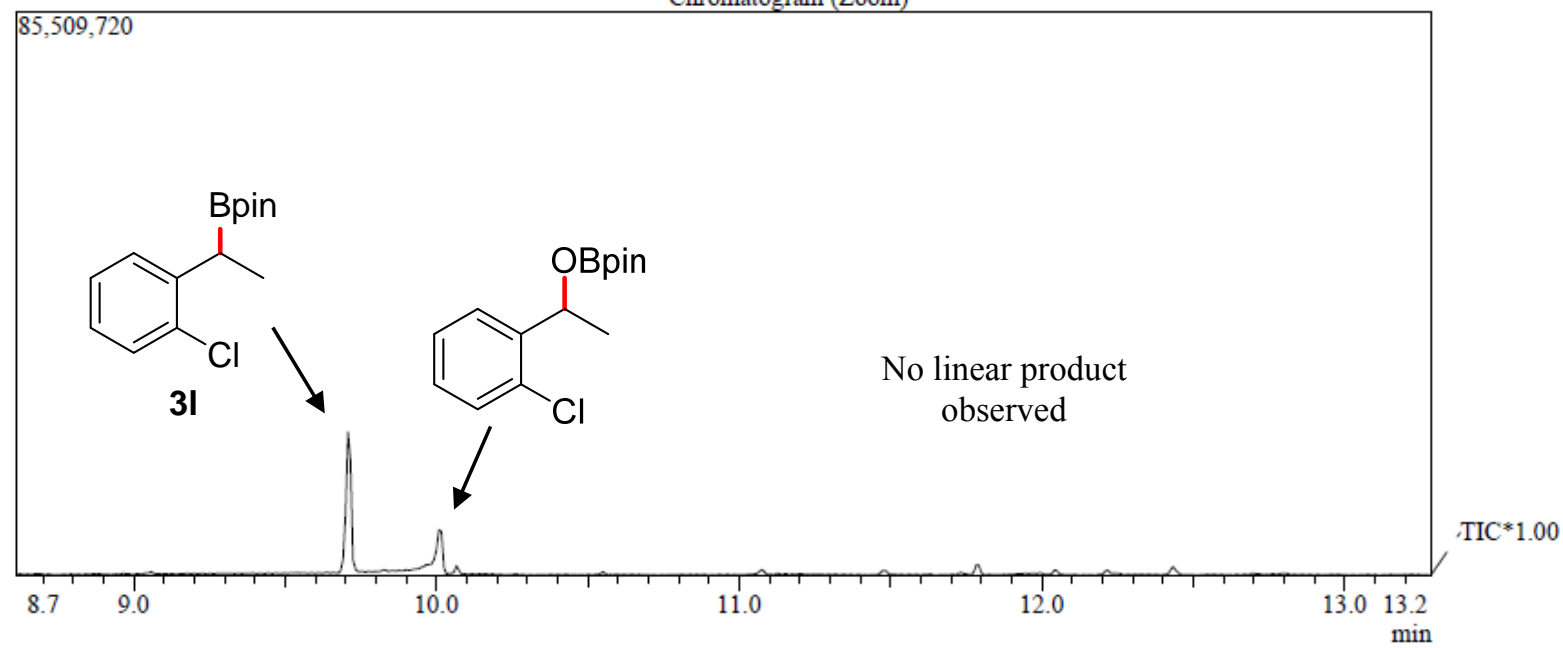

Line\#:1 R.Time:9.708(Scan\#:1165)

MassPeaks:431

RawMode:Single 9.708(1165) BasePeak:83.05(1979955)

BG Mode:None

b)

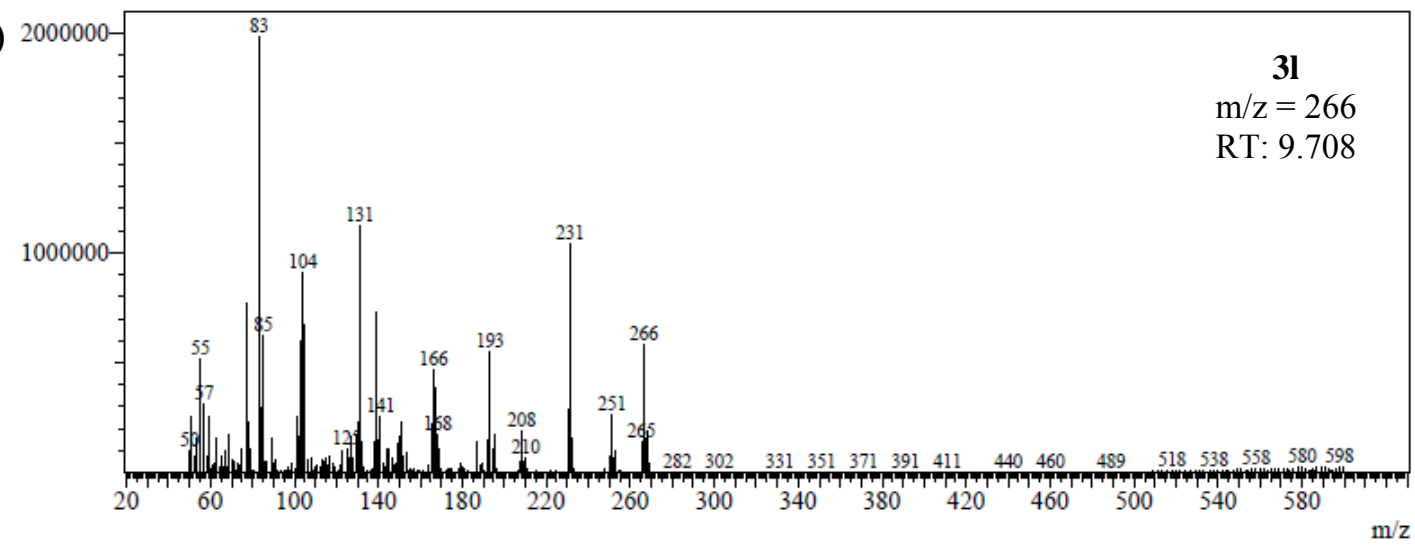

Line\#:1 R.Time:10.008(Scan\#:1201)

MassPeaks:476

RawMode:Single 10.008(1201) BasePeak:139.00(607436)

BG Mode:None

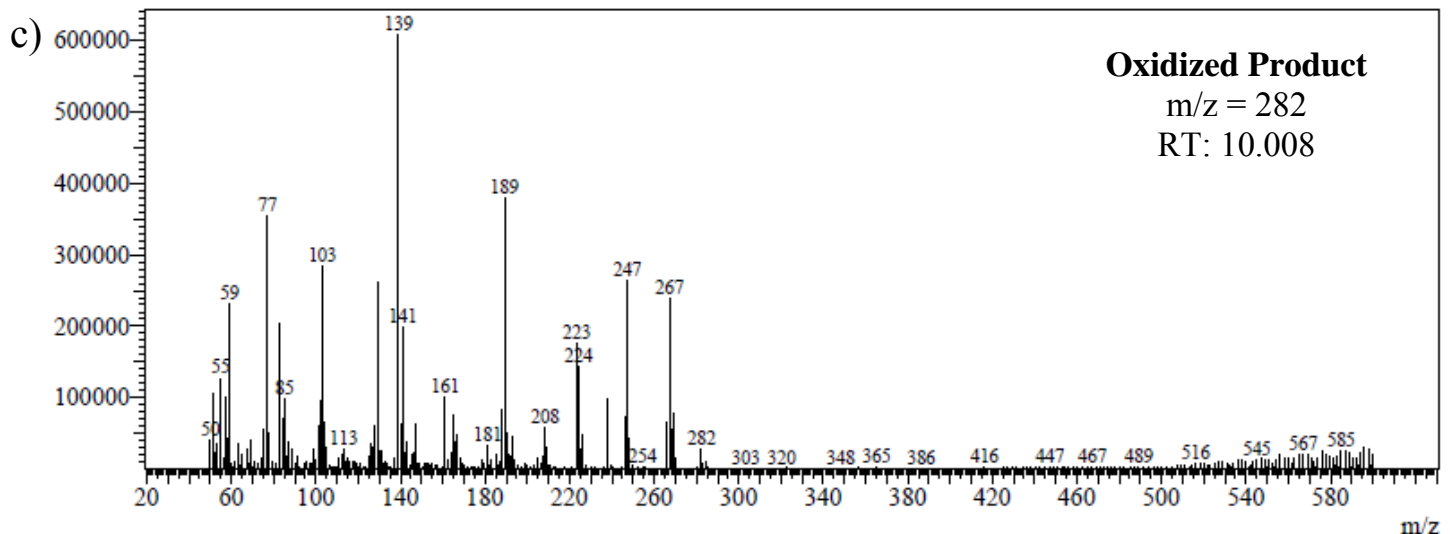

Figure S21. GC-MS chromatograms of product 3l and oxidized product. Assignment of oxidized product is tentative. 
Chromatogram (Zoom)

a)

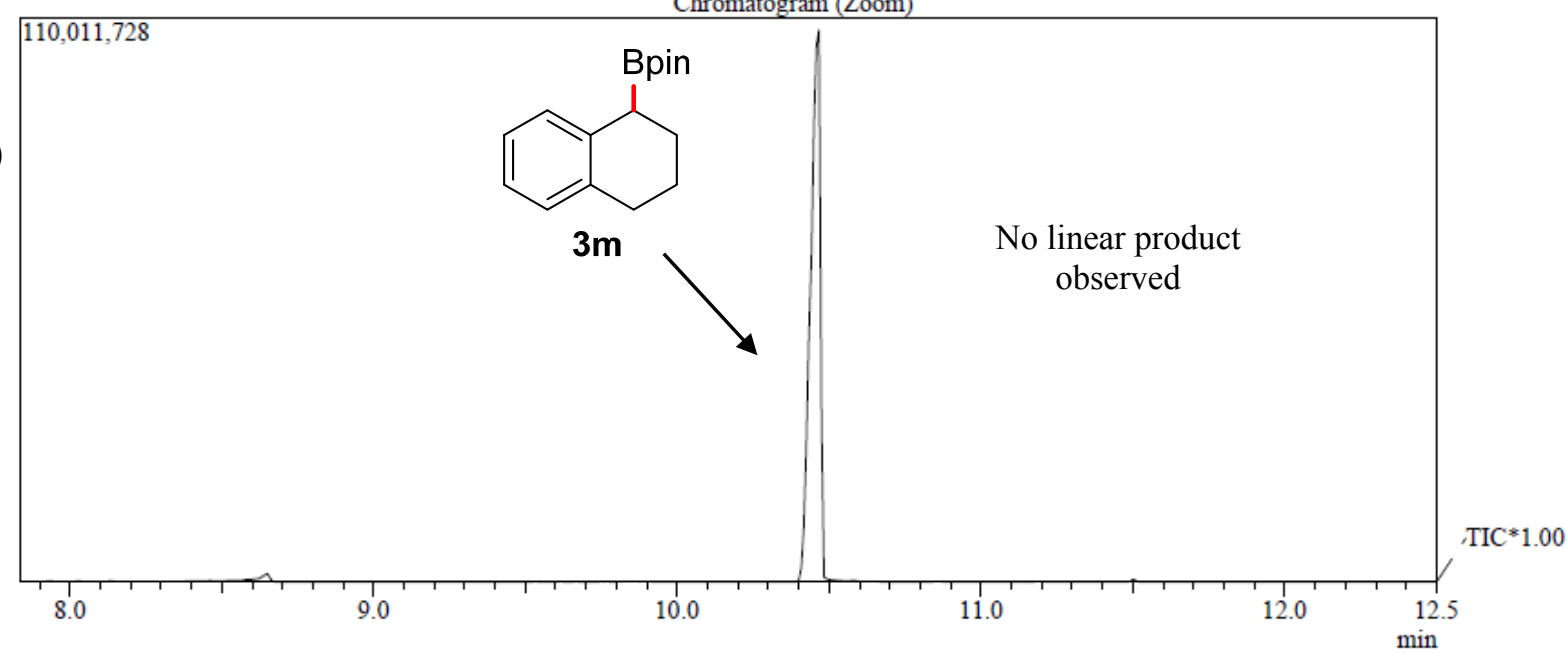

Line\#:1 R.Time:10.450(Scan\#:1254)

MassPeaks:428

RawMode:Single 10.450(1254) BasePeak:130.15(15512546)

BG Mode:None

b)

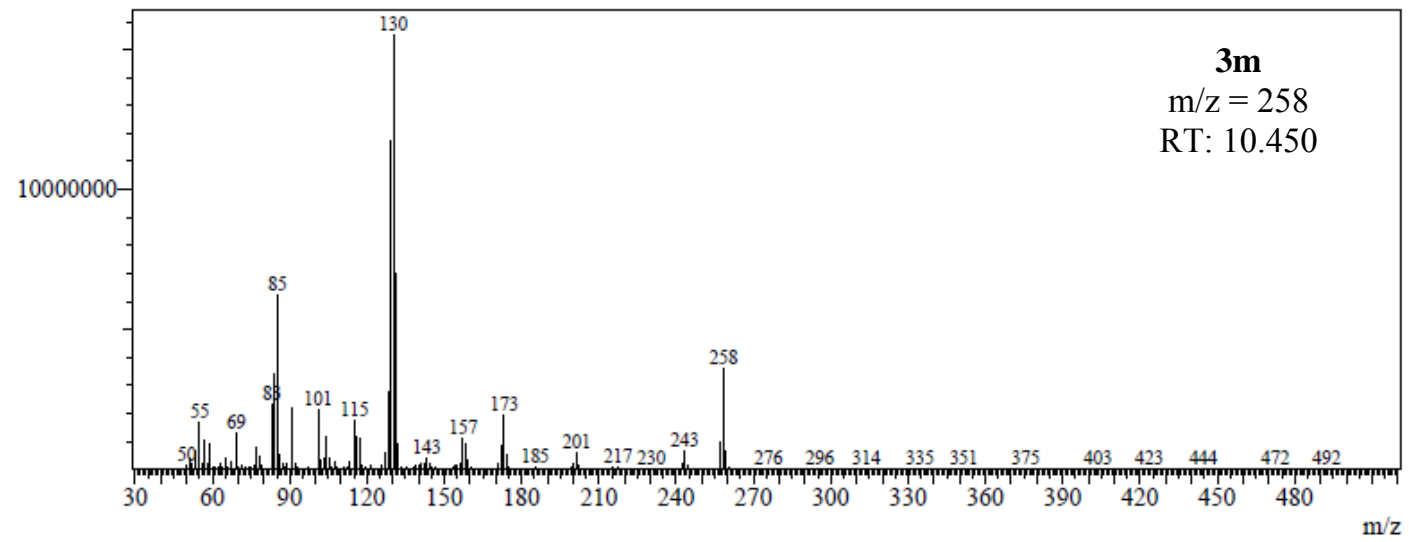

Figure S22. GC-MS chromatograms of product $\mathbf{3 m}$. 
Chromatogram (Zoom)

a)

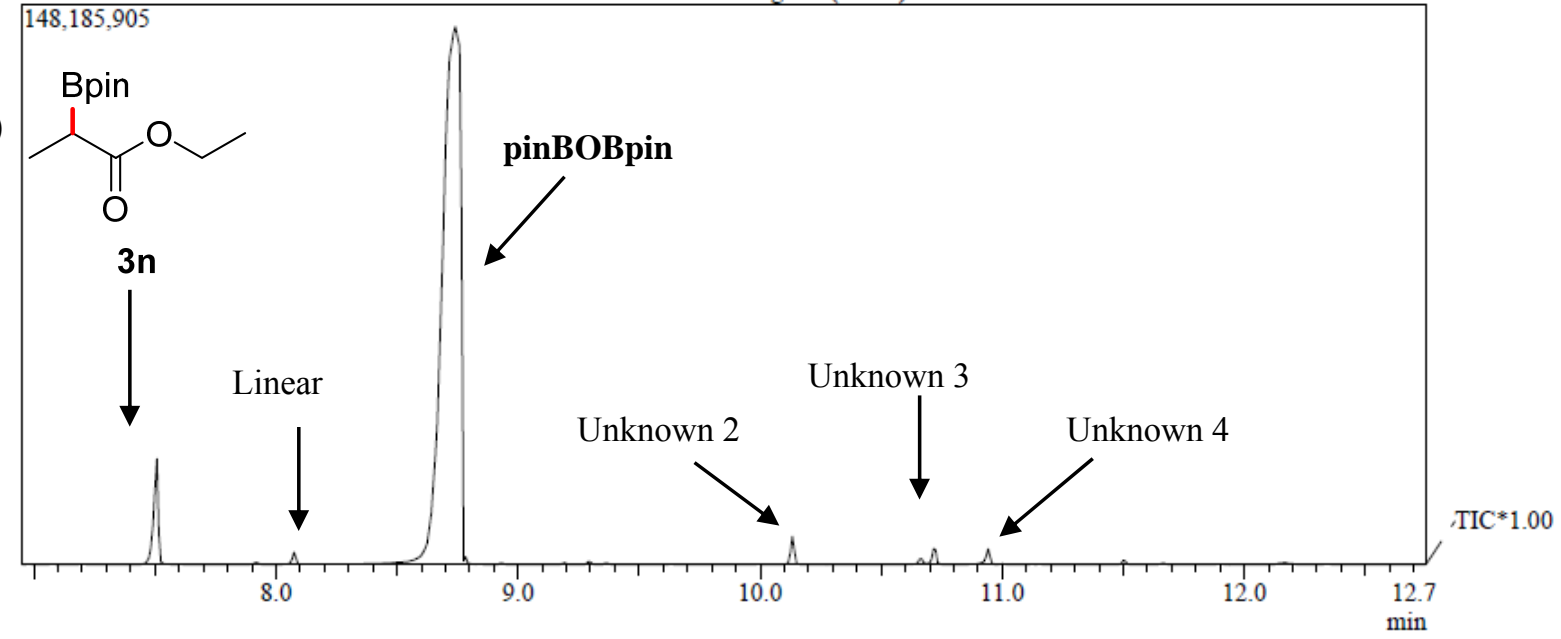

Line\#:1 R.Time:7.508(Scan\#:782)

MassPeaks: 437

RawMode:Single 7.508(782) BasePeak:55.00(2489137)

BG Mode:None

b)

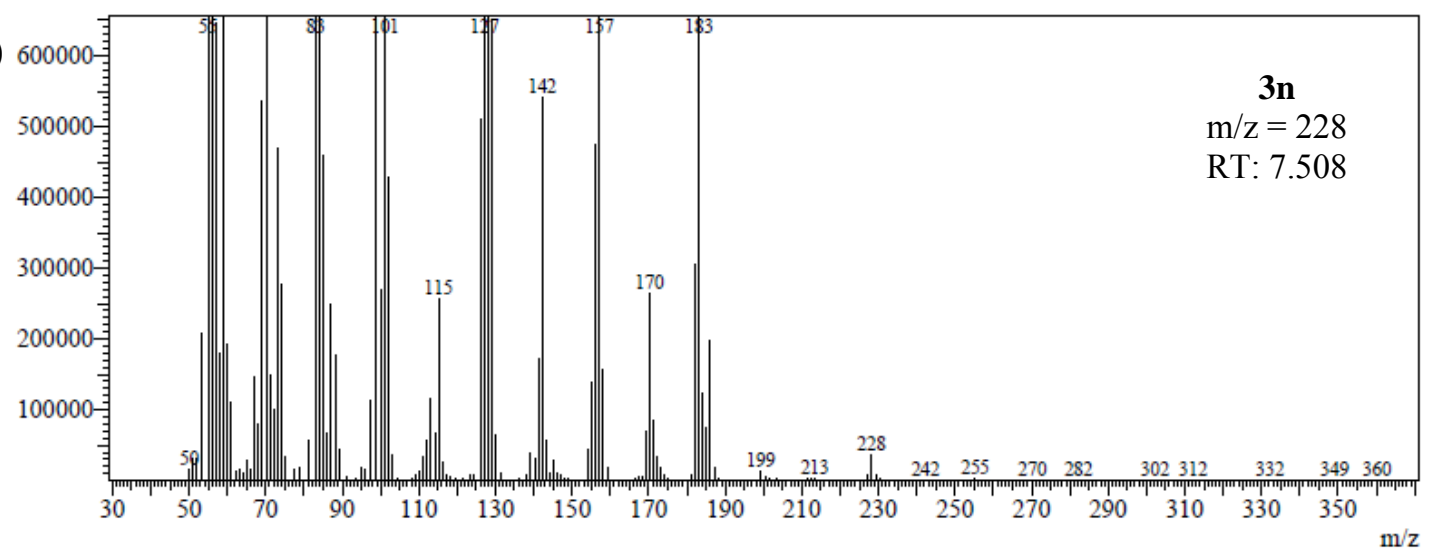

Line\#:1 R.Time:7.508(Scan\#:782)

MassPeaks:437

RawMode:Single 7.508(782) BasePeak:55.00(2489137)

BG Mode:None

c)

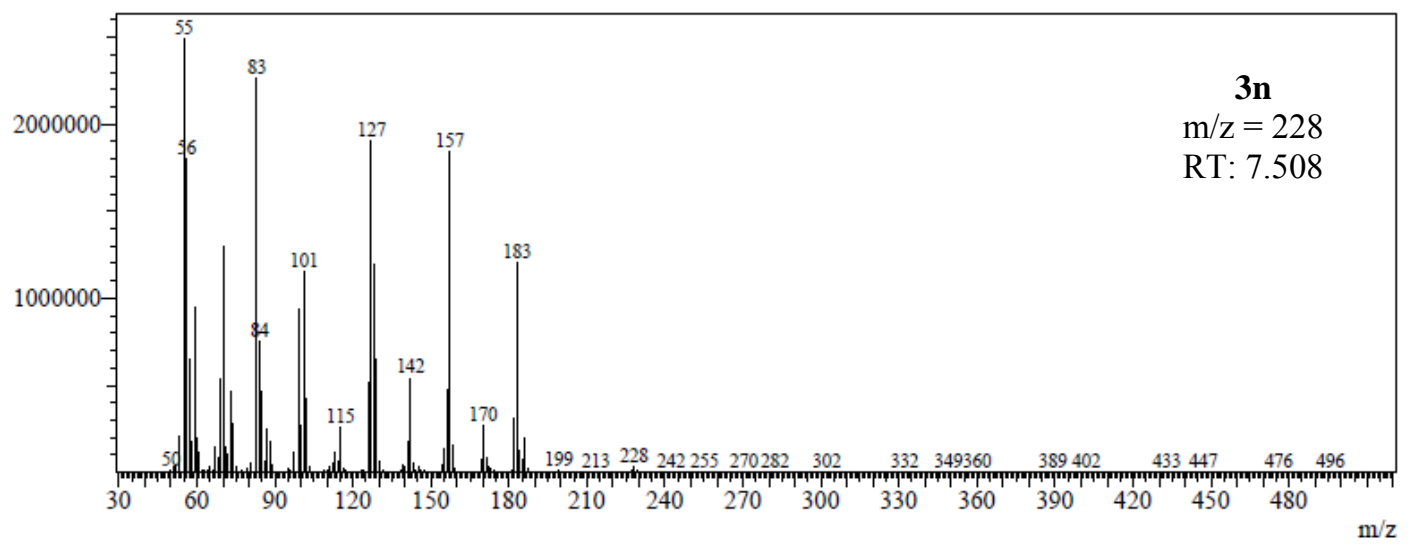


Line\#:1 R.Time:8.067(Scan\#:849)

MassPeaks: 438

RawMode:Single 8.067(849) BasePeak:171.20(152277)

BG Mode:None

d)

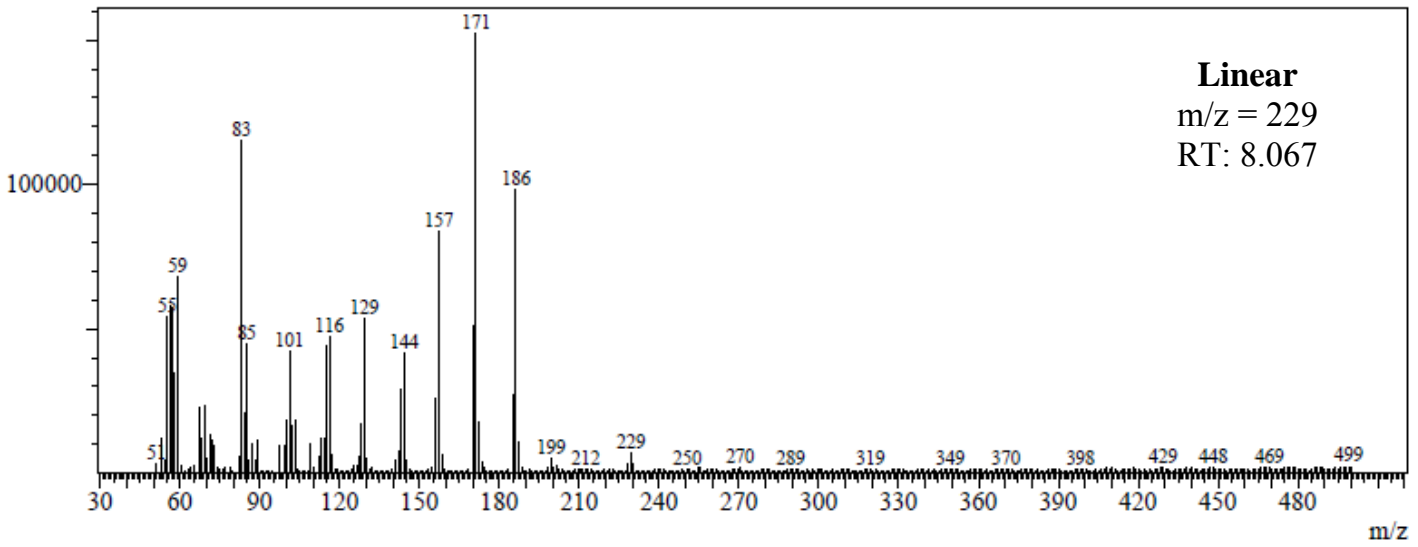

Line\#:1 R.Time:8.742(Scan\#:930)

MassPeaks: 425

RawMode:Single 8.742(930) BasePeak:255.20(10760149)

BG Mode:None

e) 10000000

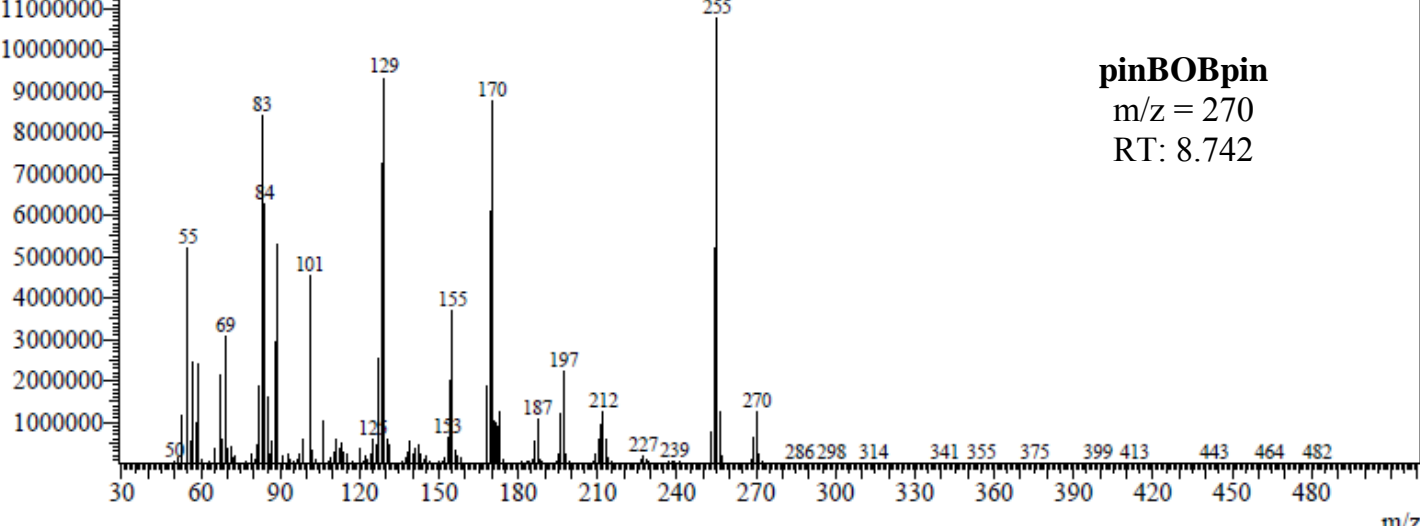

Line\#:1 R.Time:10.142(Scan\#:1098)

MassPeaks:435

RawMode:Single 10.142(1098) BasePeak:69.10(436505)

BG Mode:None

f)

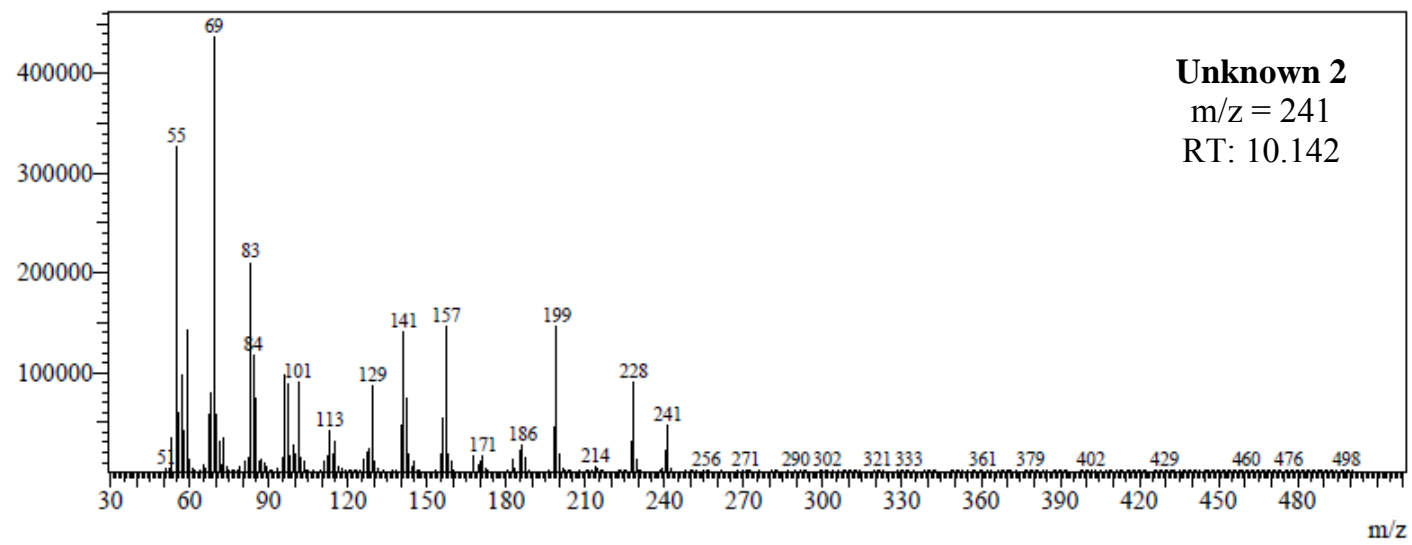


Line\#:1 R.Time:10.717(Scan\#:1167)

MassPeaks: 429

RawMode:Single 10.717(1167) BasePeak:171.15(427174)

BG Mode:None

g)

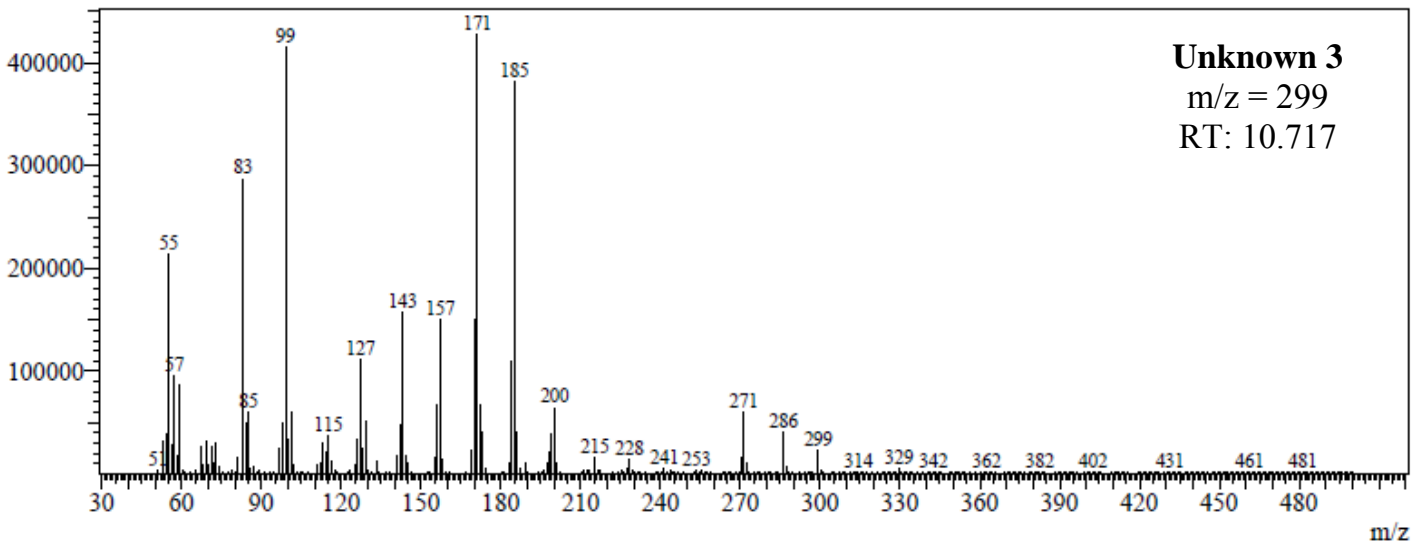

Line\#-1 R Time-10.942(Scan\#:1194)

MassPeaks: 426

RawMode:Single 10.942(1194) BasePeak:84.10(724543)

BG Mode:None

h)

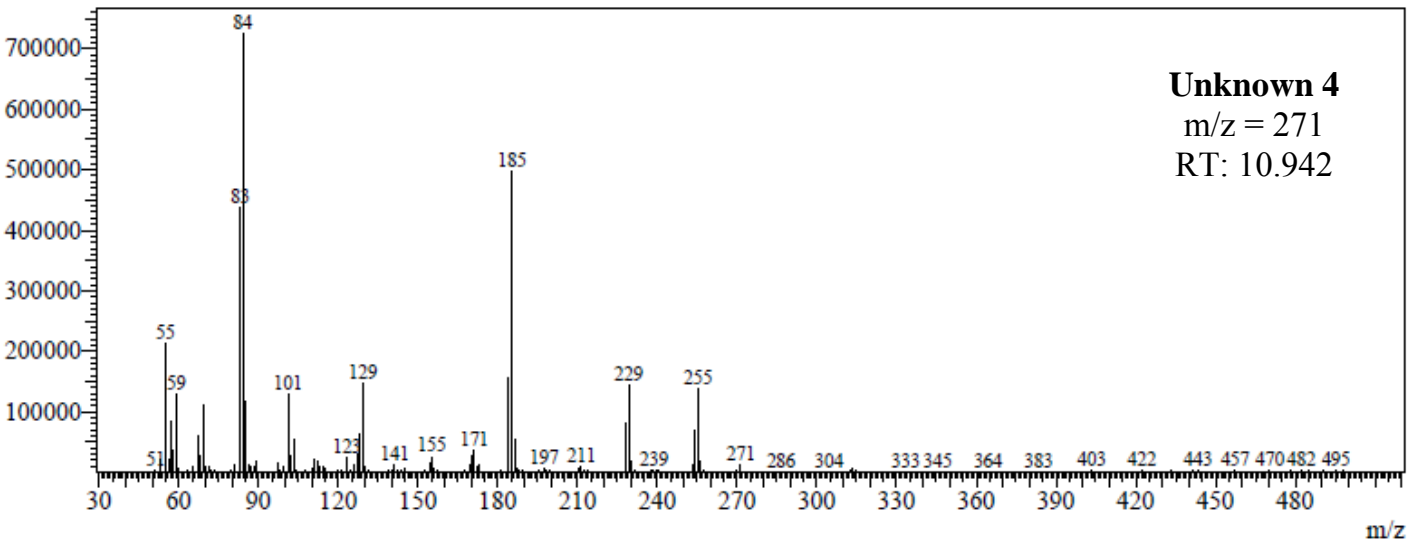

Figure S23. GC-MS chromatograms of product 3n, linear isomer, pinBOBpin, and unknowns. 


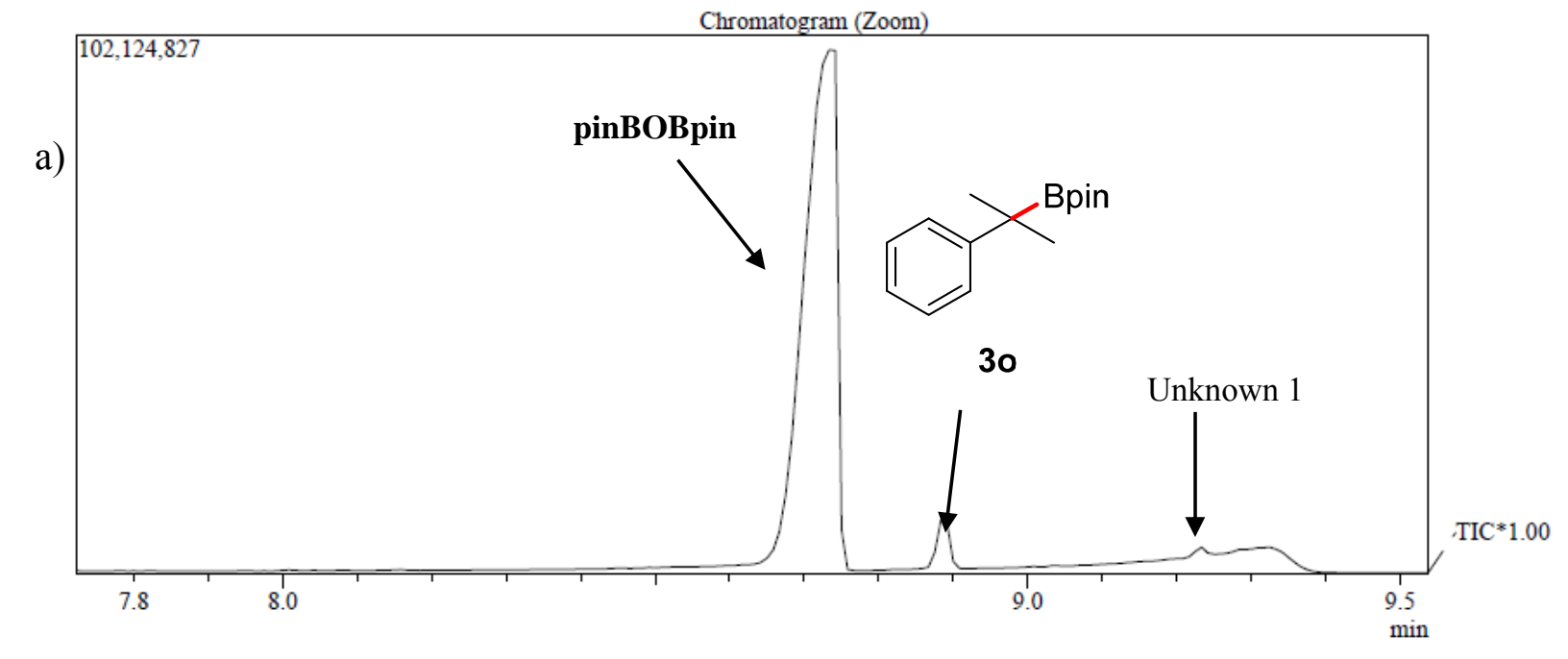

Line\#:1 R.Time:8.733(Scan\#:1048)

MassPeaks:326

RawMode:Single 8.733(1048) BasePeak:83.10(7360378)

BG Mode:Averaged 8.150-8.650(978-1038)

b)

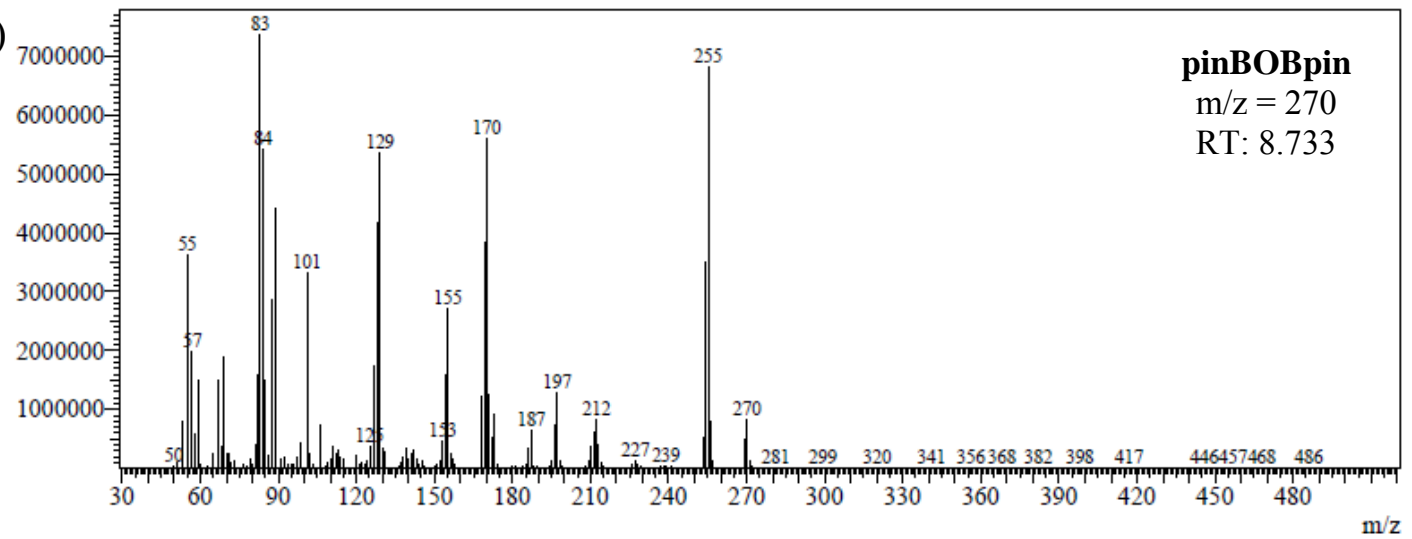

Line\#:1 R.Time:8.892(Scan\#:1067)

MassPeaks: 429

RawMode:Single 8.892(1067) BasePeak:83.05(543948)

BG Mode:None

c)

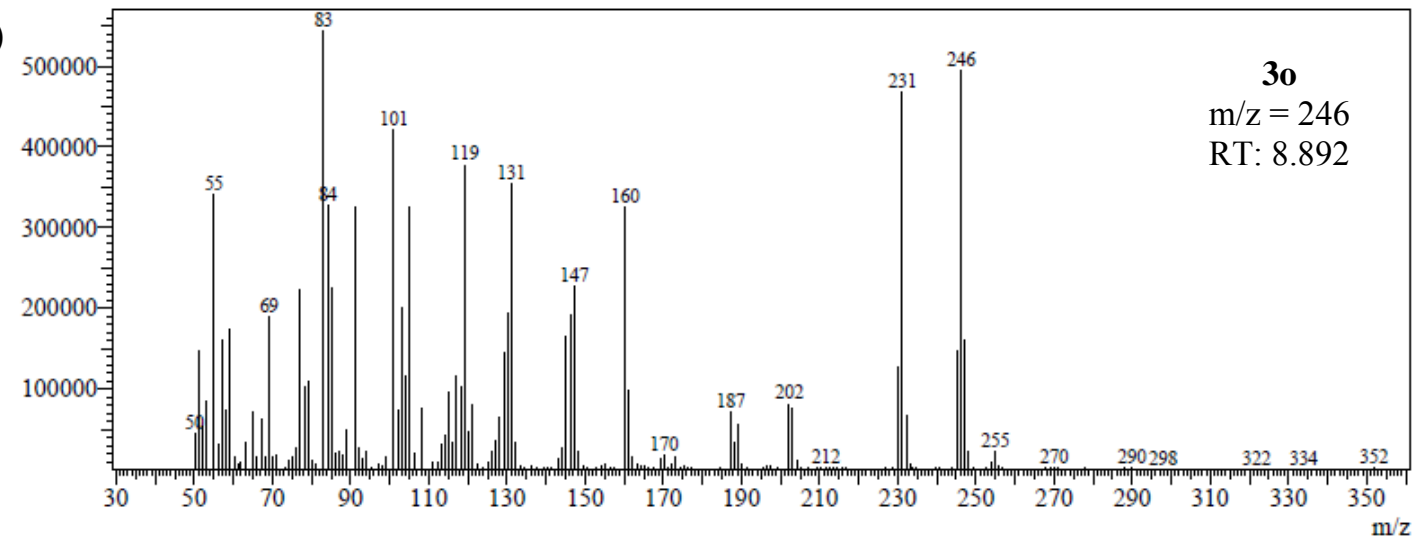




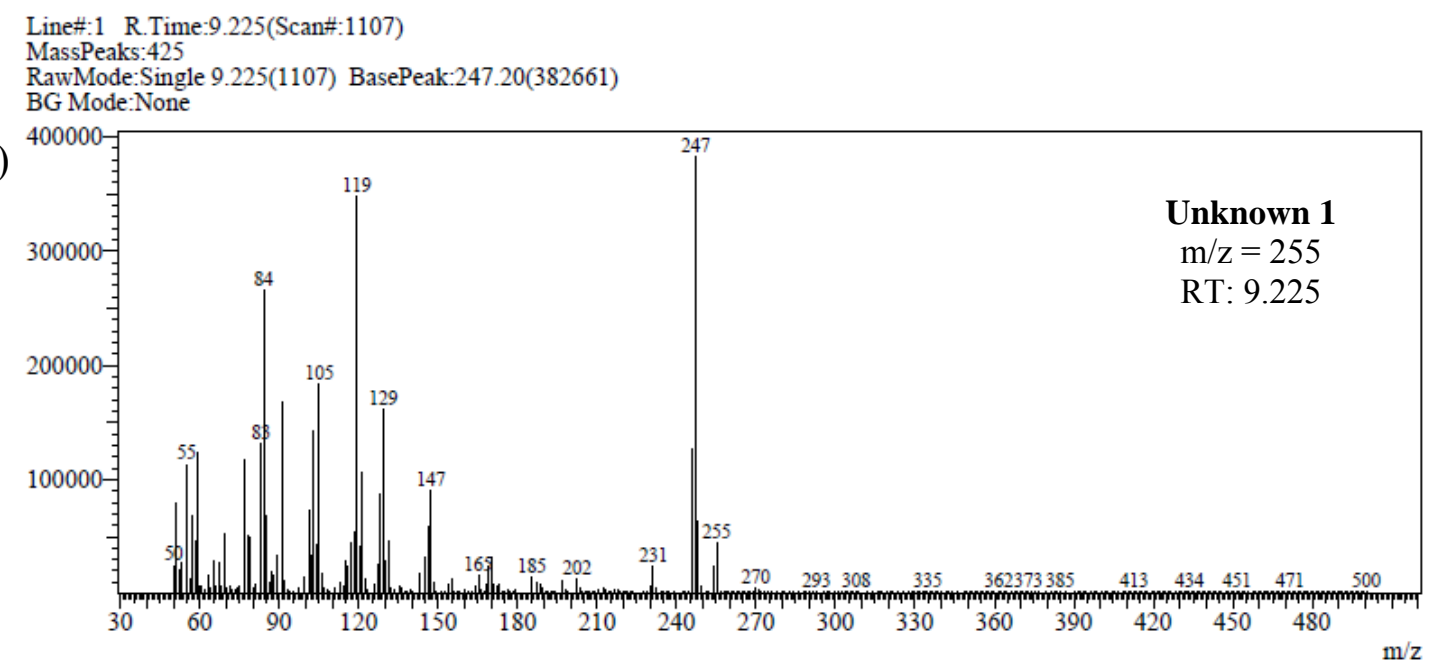

Figure S24. GC-MS chromatograms of product 3o, pinBOBpin, and an unknown. 


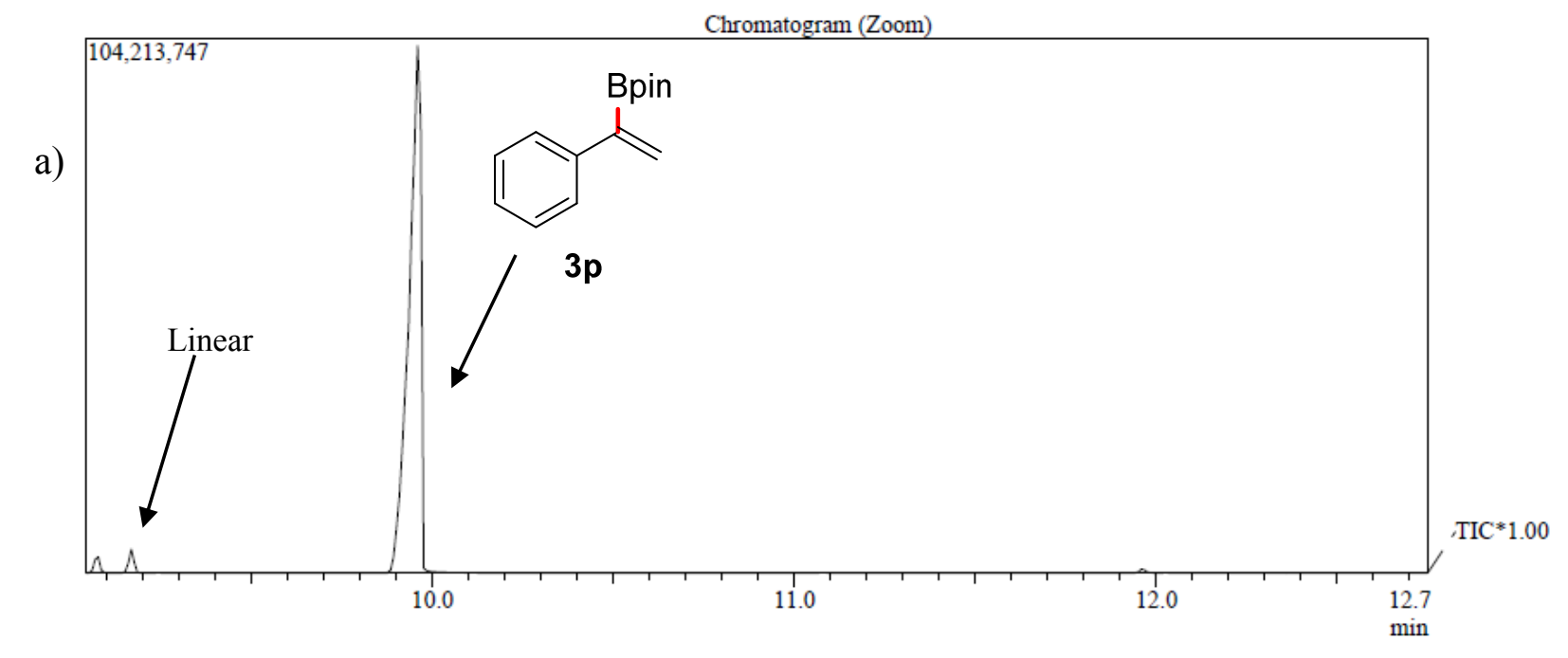

Line\#:1 R.Time:9.950(Scan\#:1075)

MassPeaks:429

RawMode:Single 9.950(1075) BasePeak:129.05(10247794)

BG Mode:None

b)

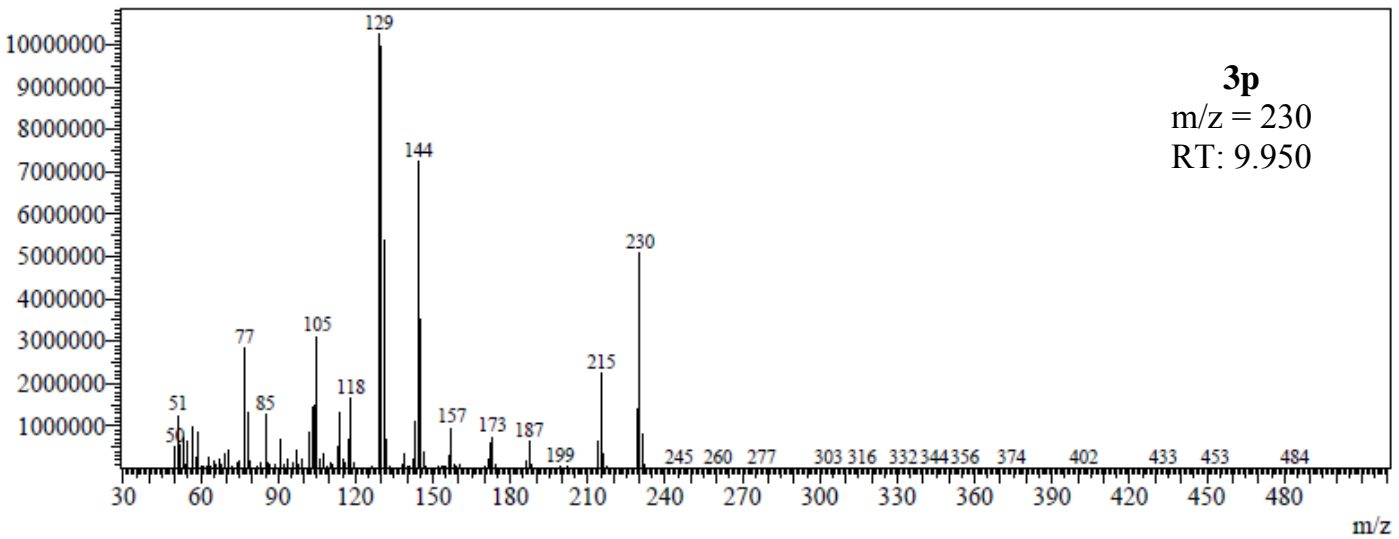

Line\#:1 R.Time:9.167(Scan\#:981)

MassPeaks:435

RawMode:Single 9.167(981) BasePeak:129.15(450981)

BG Mode:None

c)

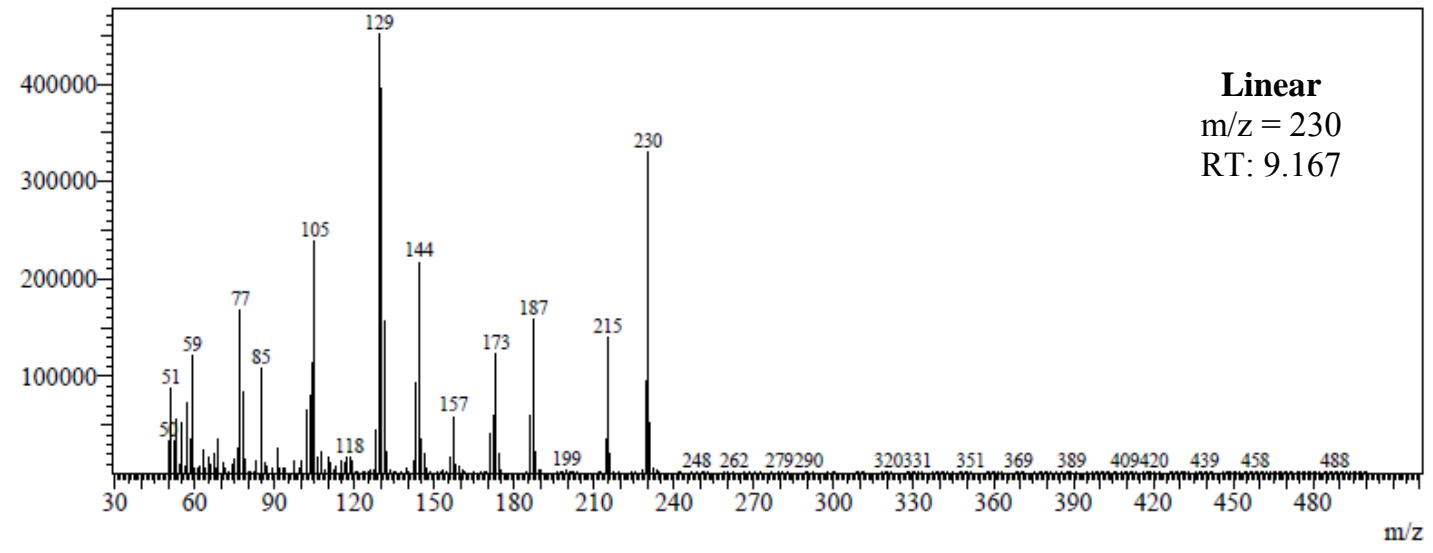

Figure S25. GC-MS chromatograms of product 3p and linear isomer. 
Chromatogram (Zoom)

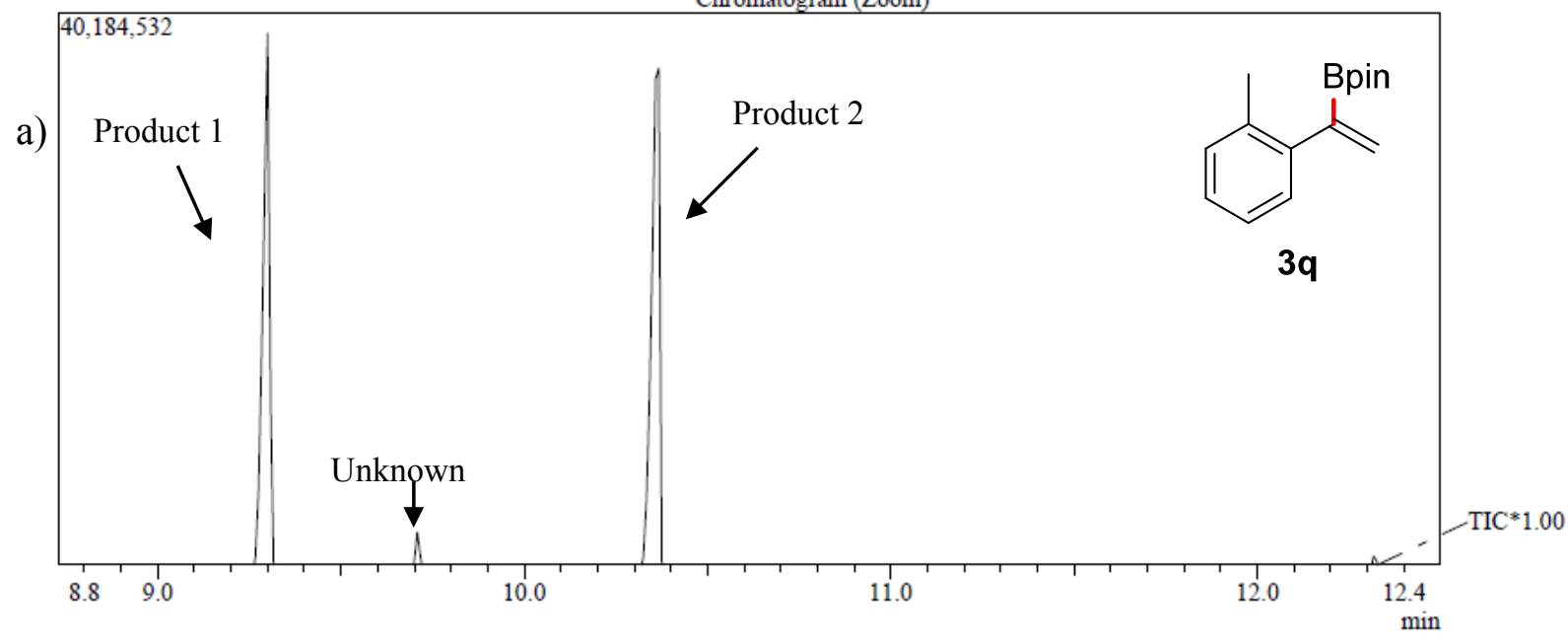

Line\#:1 R.Time-9.292(Scan\#:996)

MassPeaks: 433

RawMode:Single 9.292(996) BasePeak:144.15(4411612)

BG Mode:None

b)

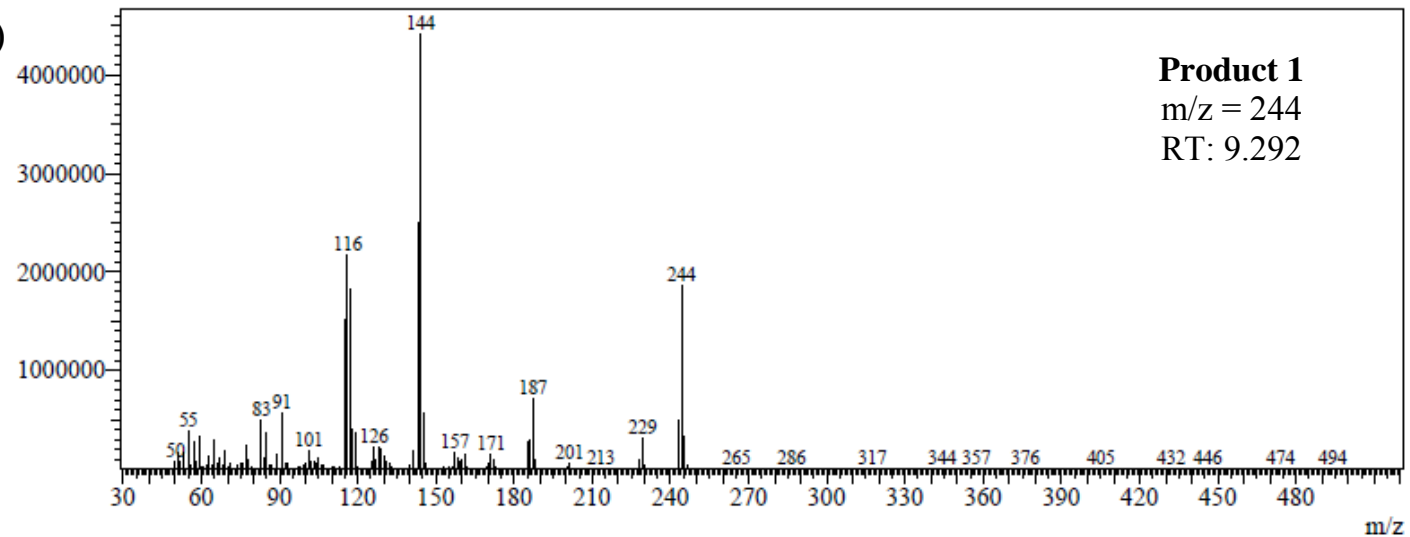

Line\#:1 R.Time:9.708(Scan\#:1046)

MassPeaks:437

RawMode:Single 9.708(1046) BasePeak:84.00(522551)

BG Mode:None

c)

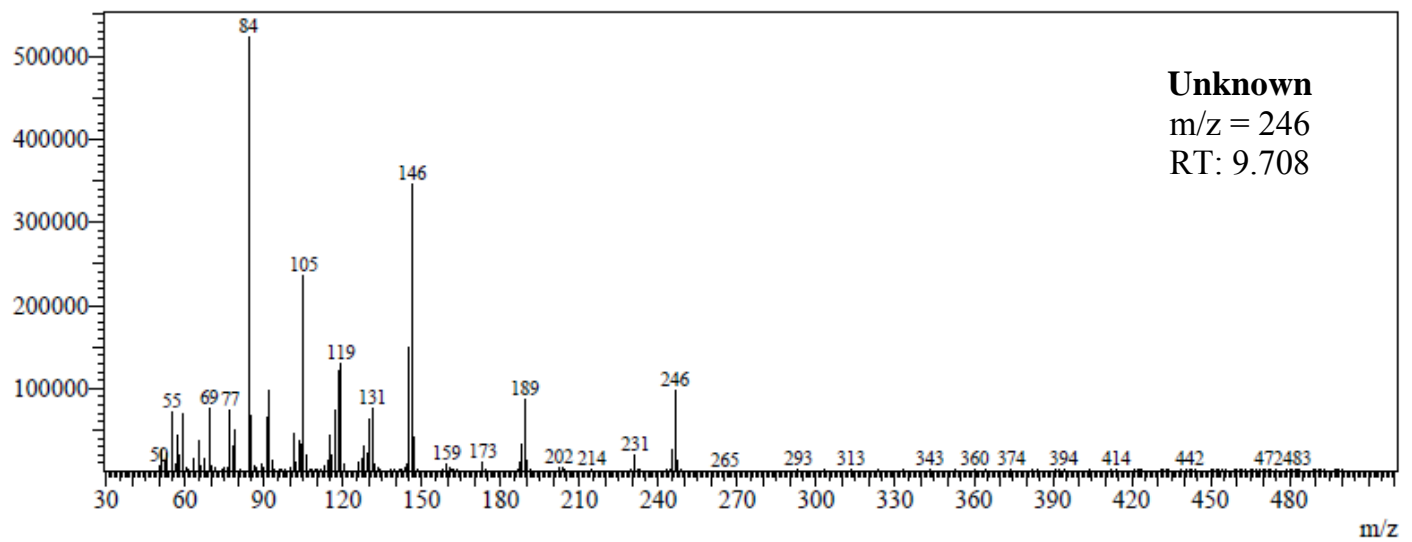


Line\#:1 R.Time:10.358(Scan\#:1124)

MassPeaks: 435

RawMode:Single 10.358(1124) BasePeak:144.15(5498231)

d)

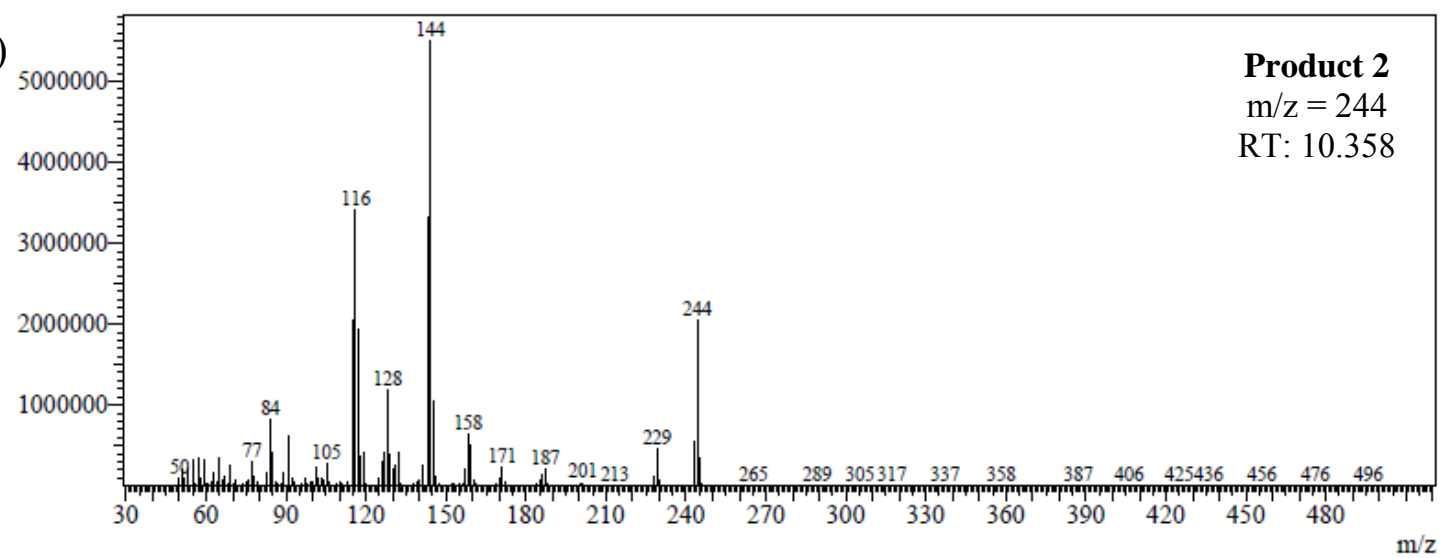

Figure S26. GC-MS chromatograms of product 3q, an isomer, and an unknown. 
Chromatogram (Zoom)

a)

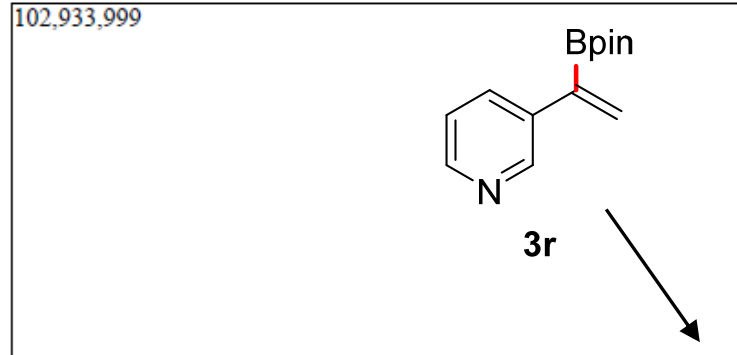

Line\#:1 R. Time:10.358(Scan\#:1243)

MassPeaks:424

RawMode:Single 10.358(1243) BasePeak:132.10(9820971)

BG Mode:None

b)

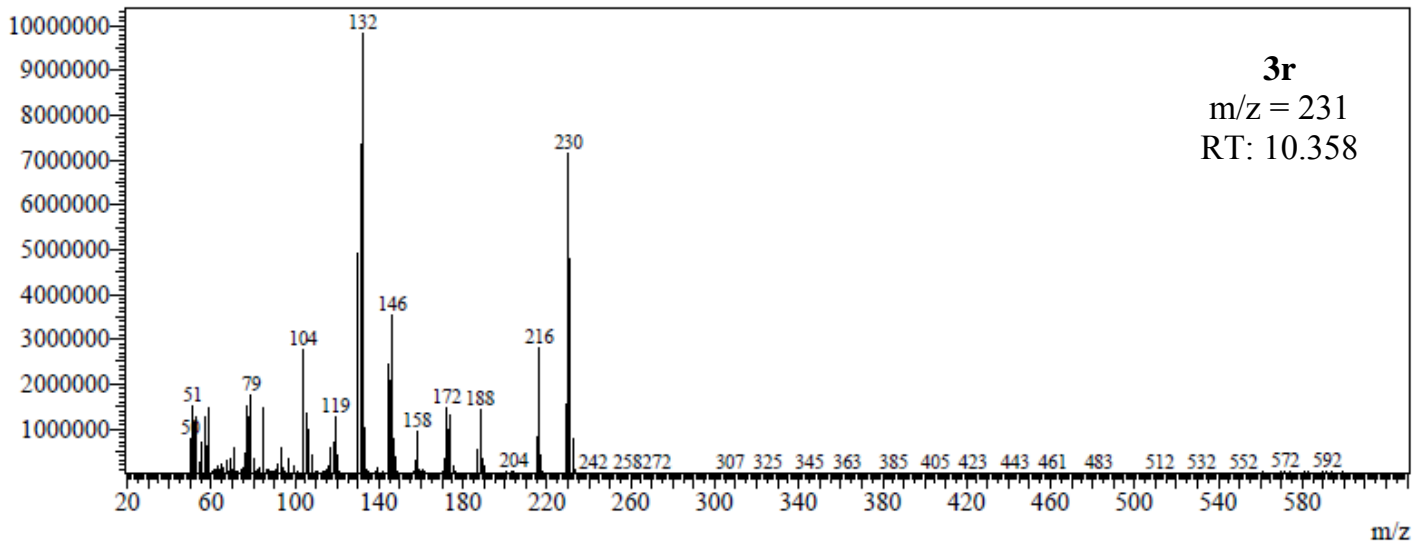

Figure S27. GC-MS chromatograms of product 3r. 
a)

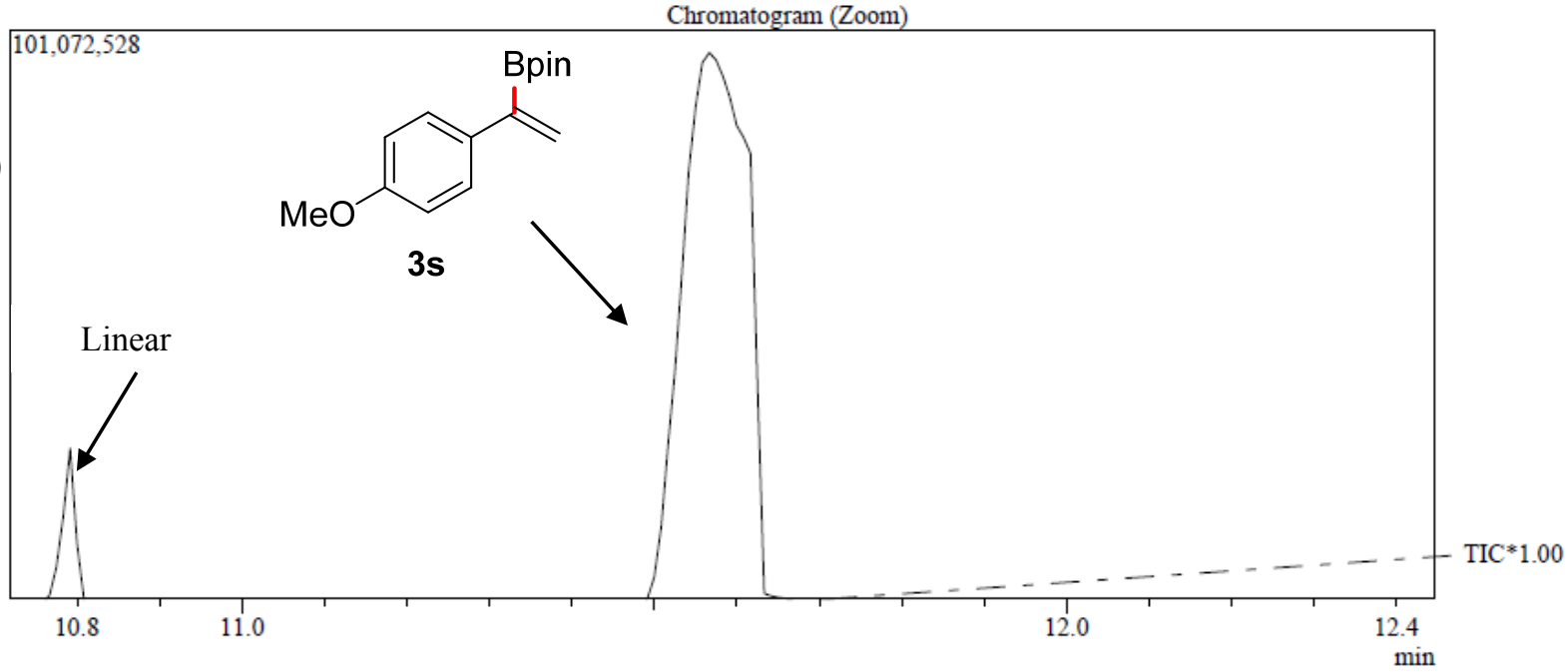

Line\#:1 R.Time:11.575(Scan\#:1389)

MassPeaks: 430

RawMode:Single 11.575(1389) BasePeak:144.15(10527417)

BG Mode:None

b)

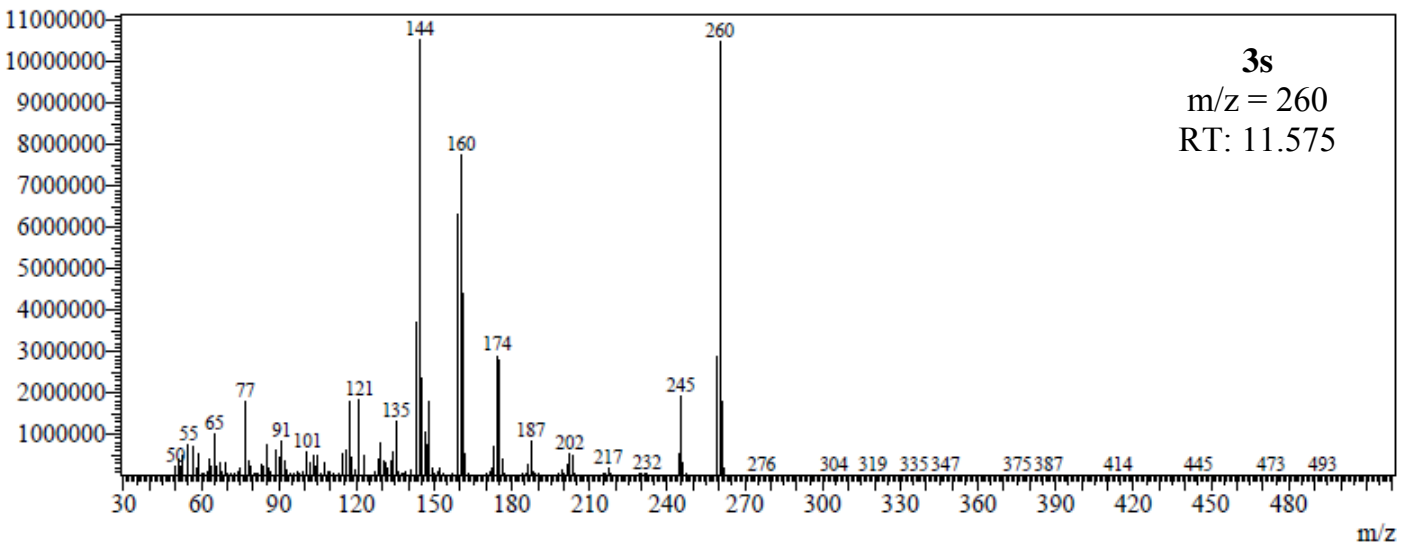

Line\#:1 R.Time:10.792(Scan\#:1295)

MassPeaks:433

RawMode:Single 10.792(1295) BasePeak:260.20(4005936)

BG Mode:None

c)

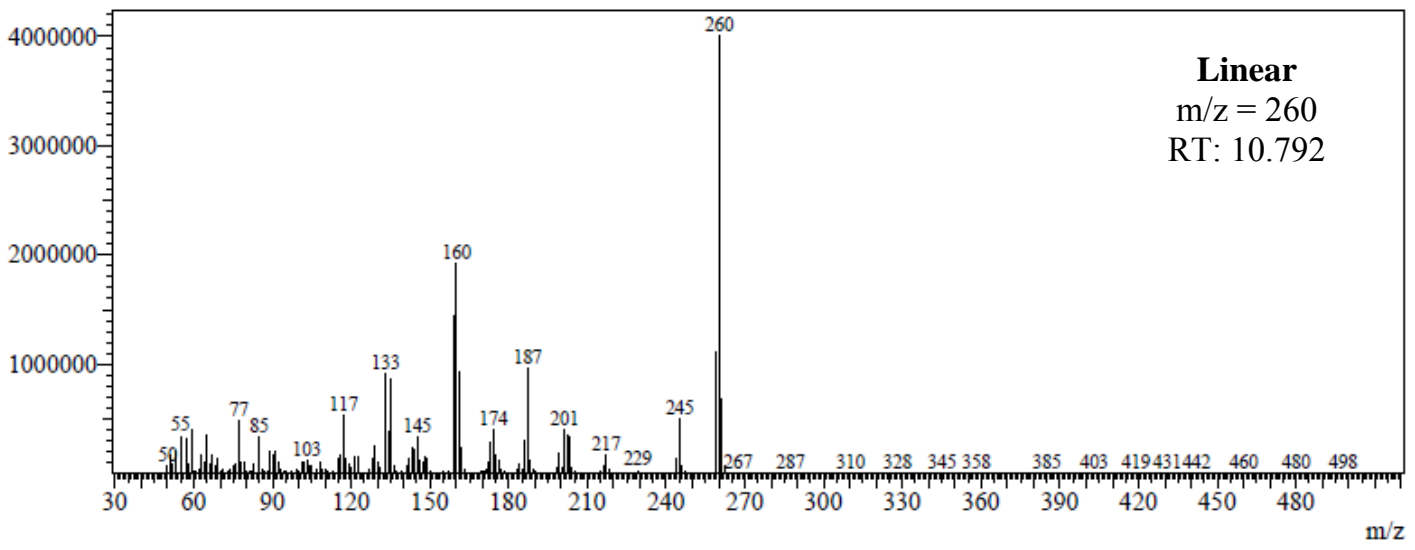

Figure S28. GC-MS chromatograms of product 3s and an isomer. 
Chromatogram (Zoom)

a)

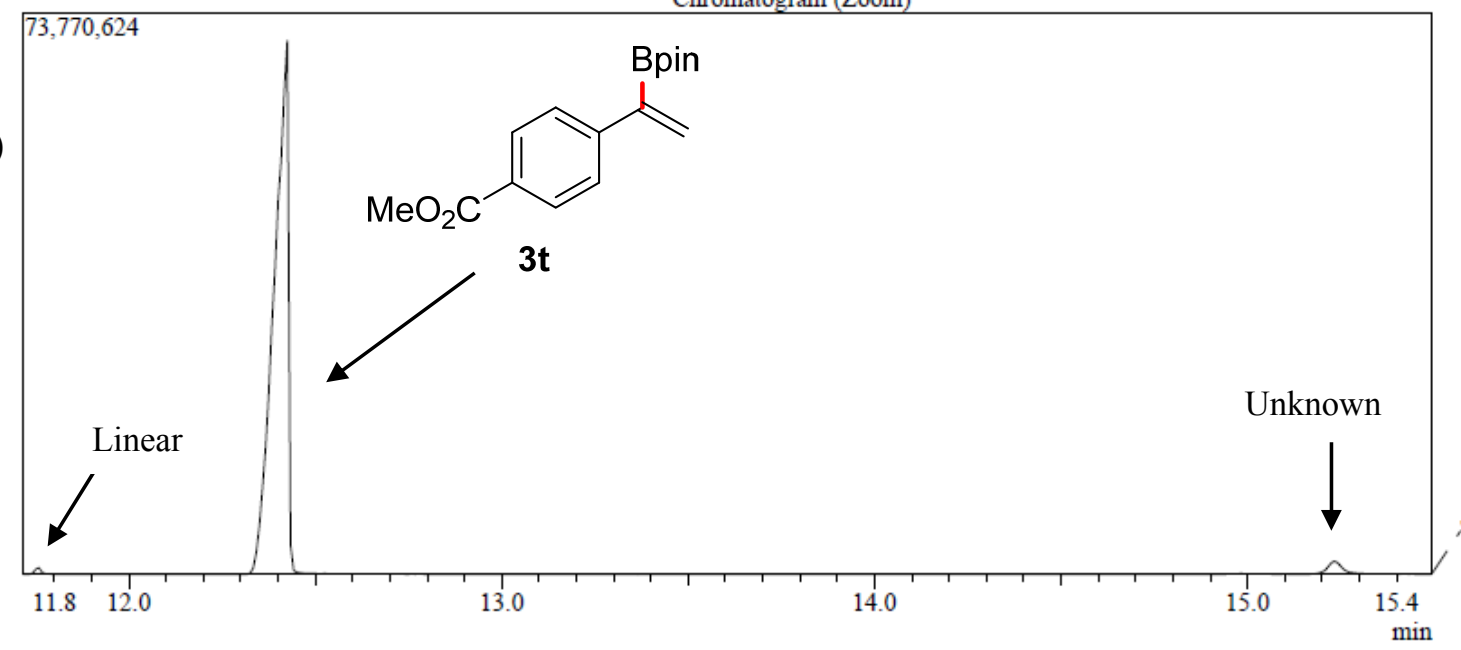

Line\#:1 R.Time: 12.425 (Scan\#:1372)

MassPeaks: 429

RawMode:Single 12.425(1372) BasePeak:157.10(6212676)

BG Mode:None

b)

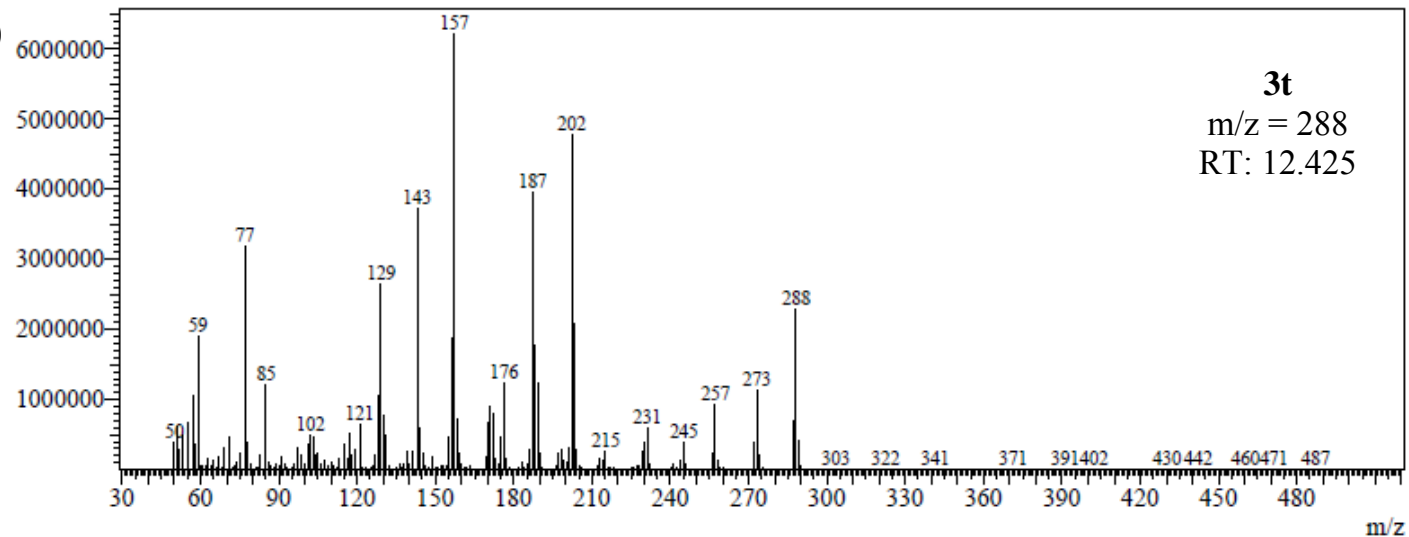

Line\#:1 R.Time: 11.750 (Scan\#:1291)

MassPeaks: 432

RawMode:Single 11.750(1291) BasePeak:157.15(52128)

BG Mode:None

c)

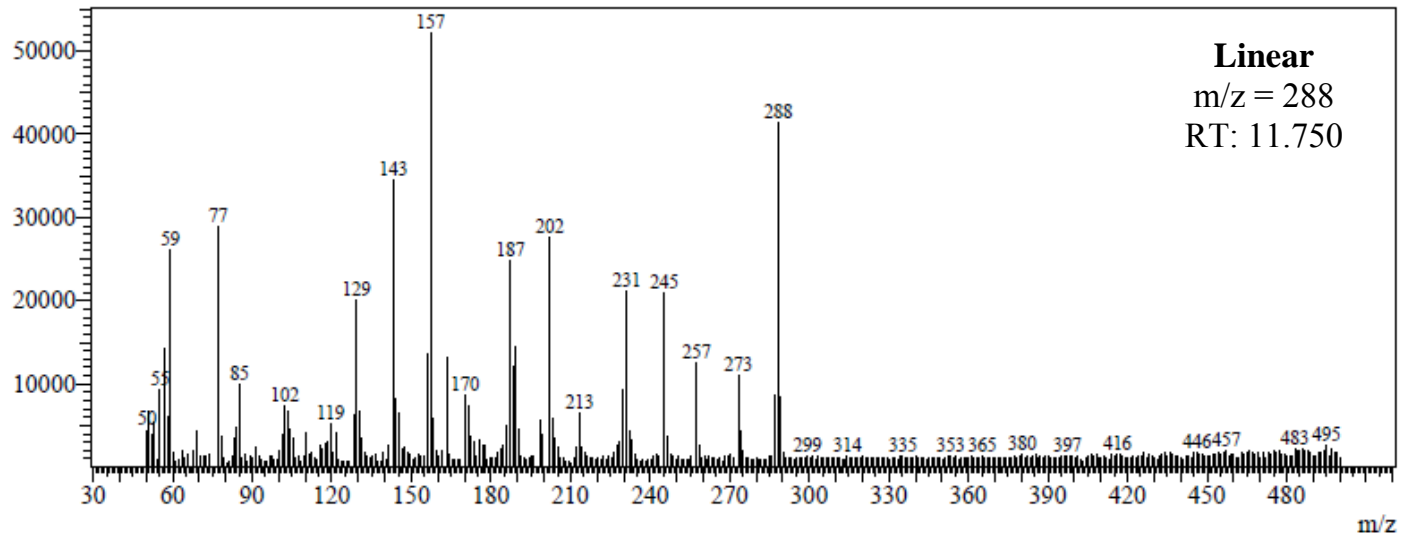


Line\#:1 R.Time:15.233(Scan\#:1709)

MassPeaks:423

RawMode:Single 15.233(1709) BasePeak:84.15(572985)

d)

ode.None

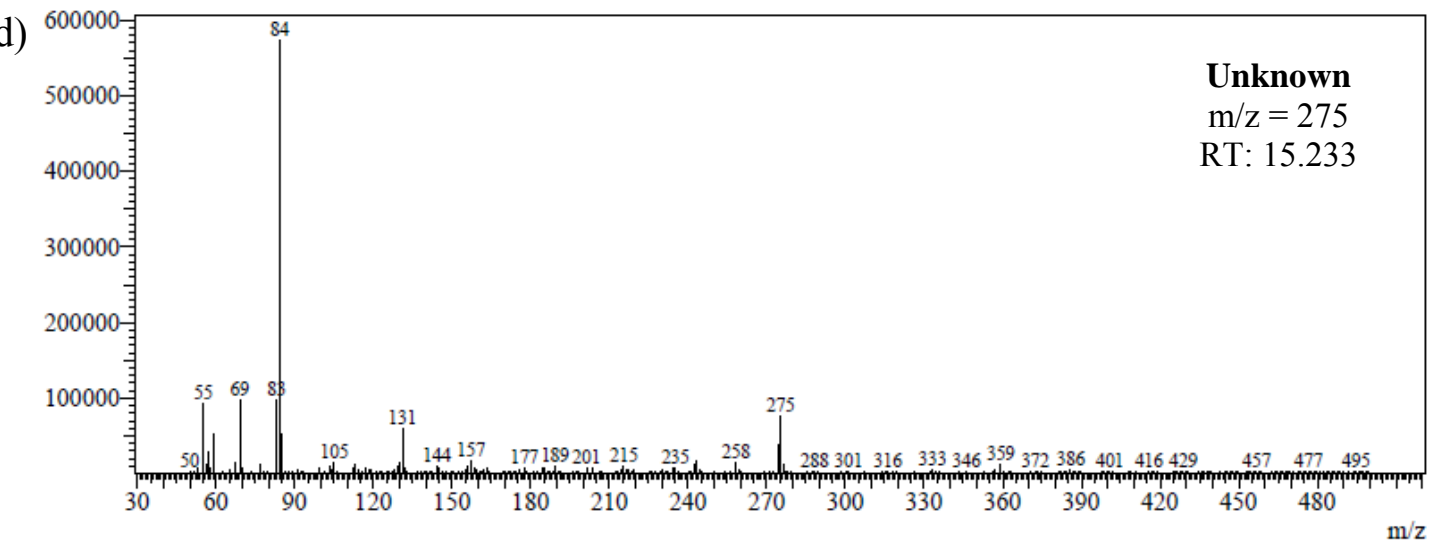

Figure S29. GC-MS chromatogram of product 3t, isomer, and unknown 
Chromatogram (Zoom)

a)

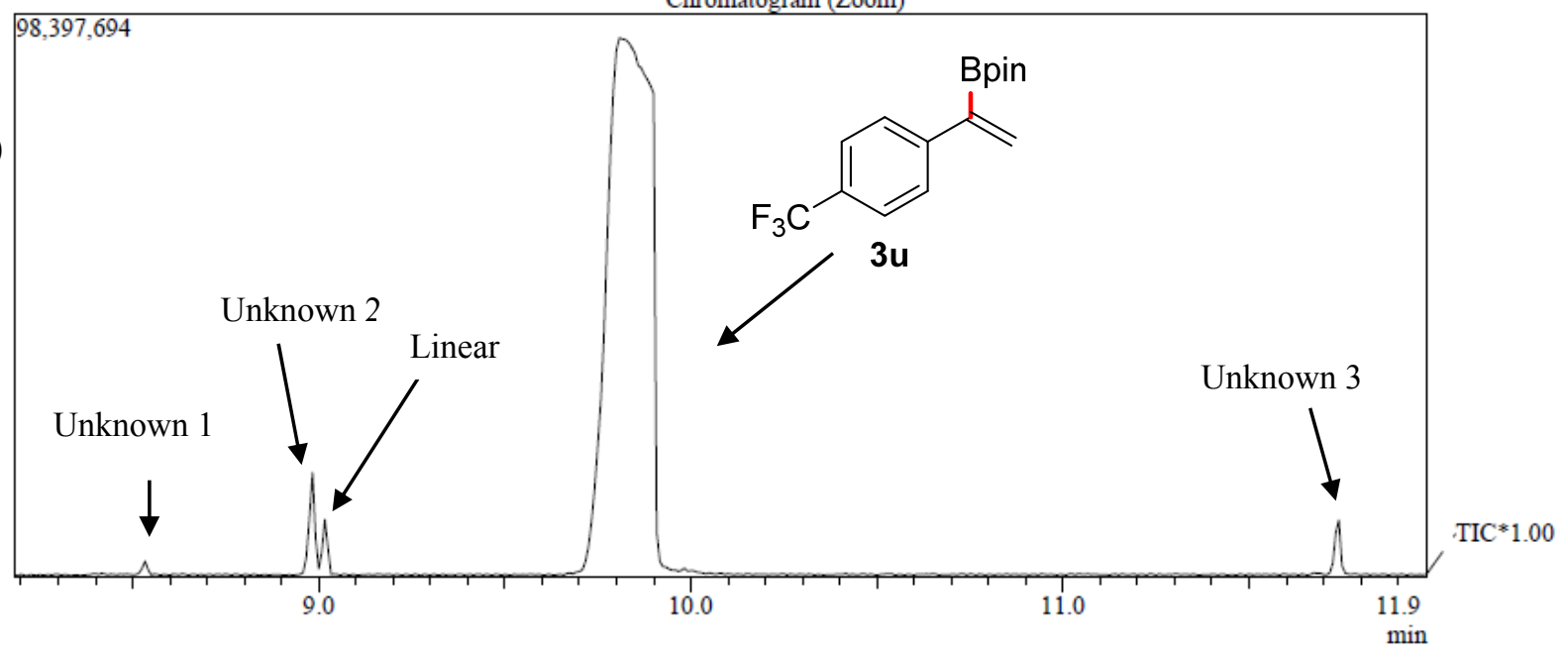

Line\#:1 R.Time:9.842(Scan\#:1181)

MassPeaks: 503

RawMode:Single 9.842(1181) BasePeak:212.05(8265192) BG Mode:None

b)

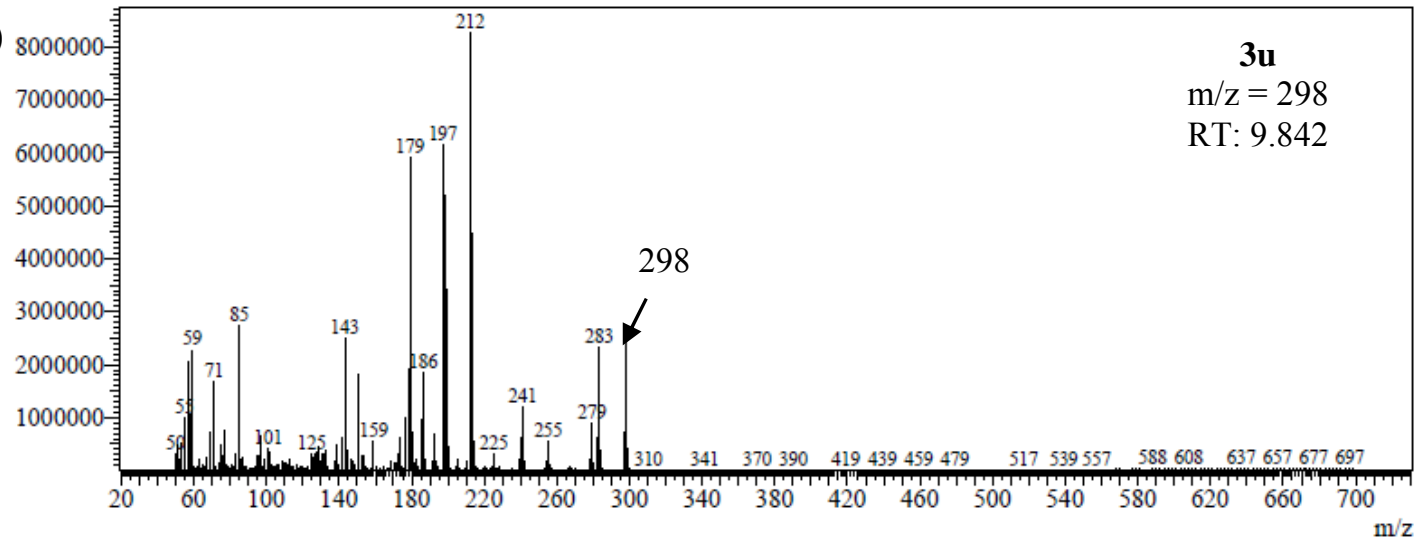

Line\#:1 R.Time:8.525(Scan\#:1023)

MassPeaks:356

RawMode:Single 8.525(1023) BasePeak:83.05(132738)

BG Mode:Averaged 8.092-8.475(971-1017)

c)

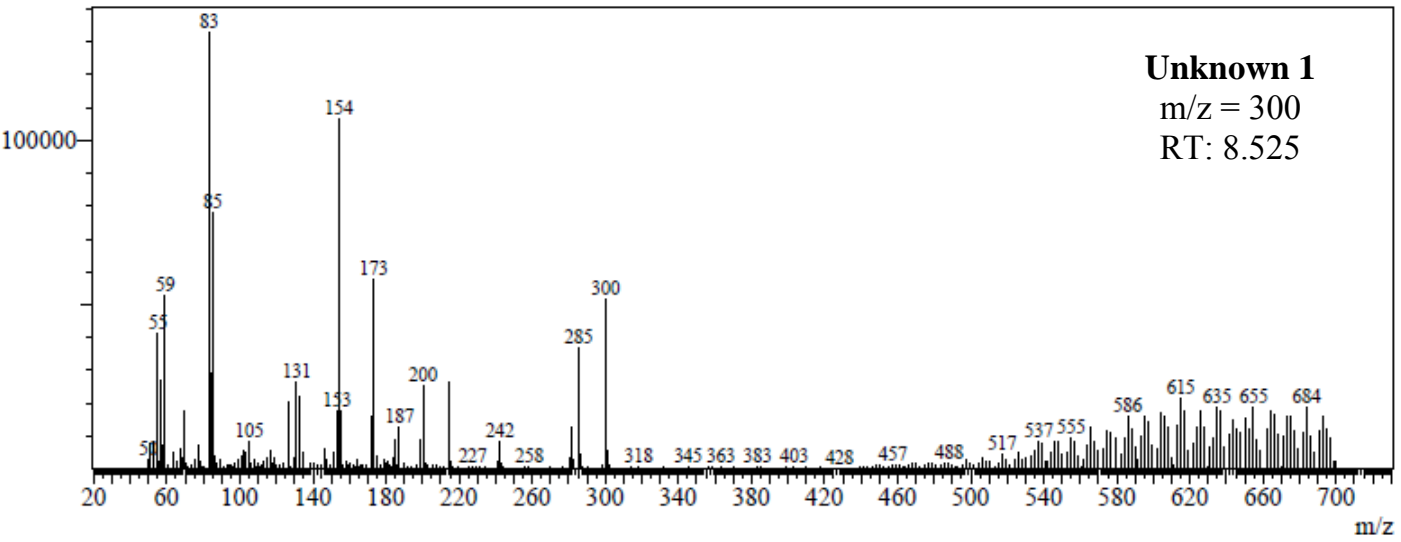


Line\#:1 R.Time:8.983(Scan\#:1078)

MassPeaks:364

RawMode:Single 8.983(1078) BasePeak:59.05(2205776)

BG Mode:Averaged 8.408-8.950(1009-1074)

d)

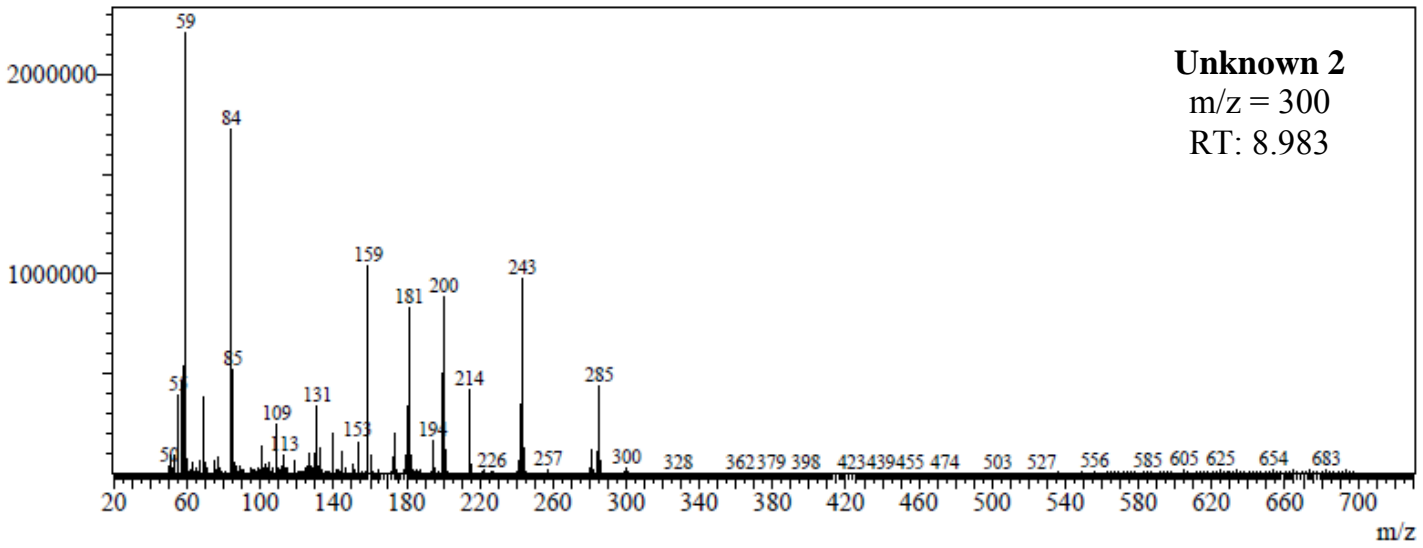

Line\#:1 R.Time:9.017(Scan\#:1082)

MassPeaks:399

RawMode:Single 9.017(1082) BasePeak:198.10(539154)

BG Mode:Averaged 8.200-8.950(984-1074)

e)

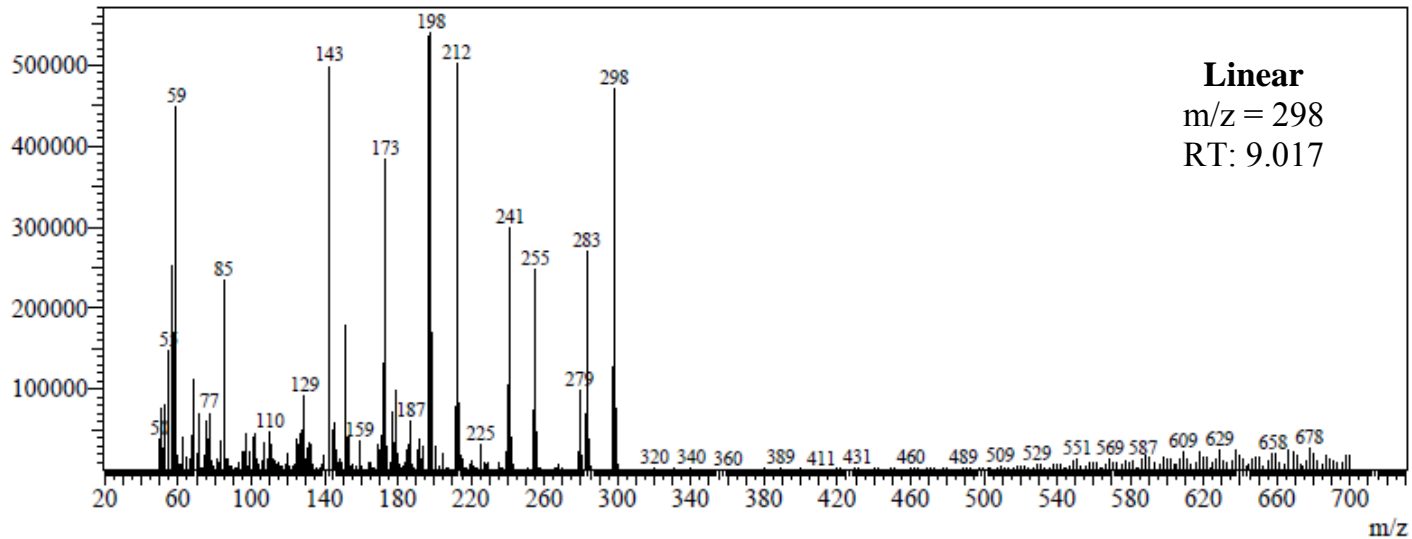

Line\#:1 R.Time:11.742(Scan\#:1409)

MassPeaks:404

RawMode:Single 11.742(1409) BasePeak:84.10(2707619)

BG Mode:Averaged 9.975-11.692(1197-1403)

f)

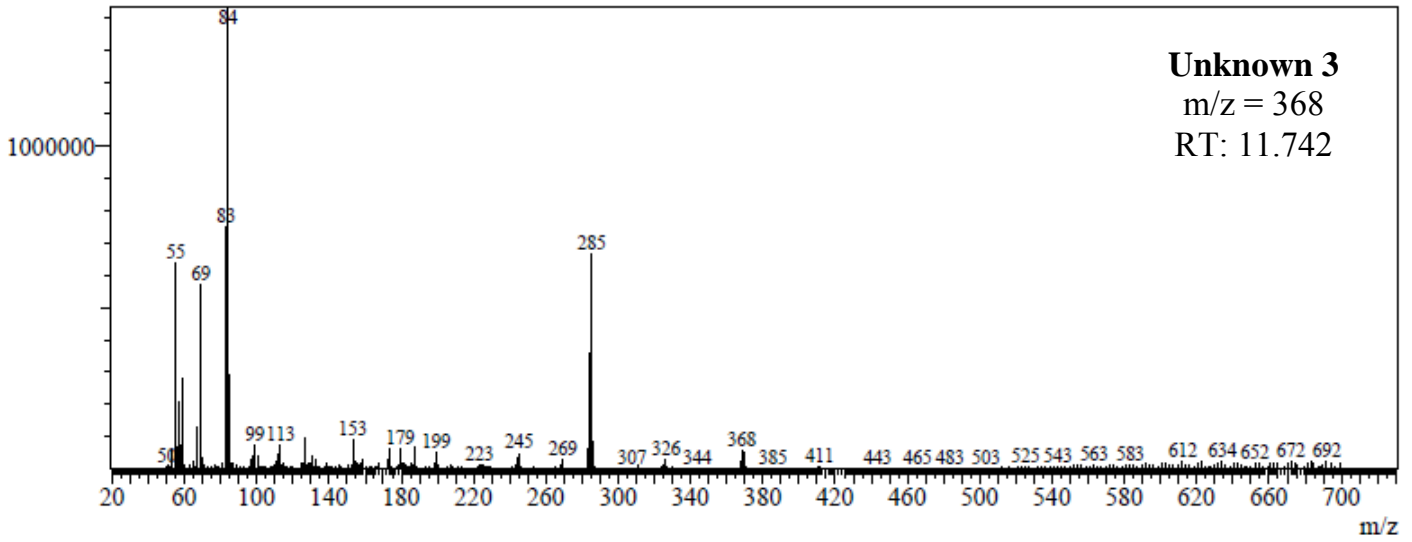

Figure S30. GC-MS chromatogram of product 3u, linear isomer and 3 unknowns. 
Chromatogram (Zoom)

a)

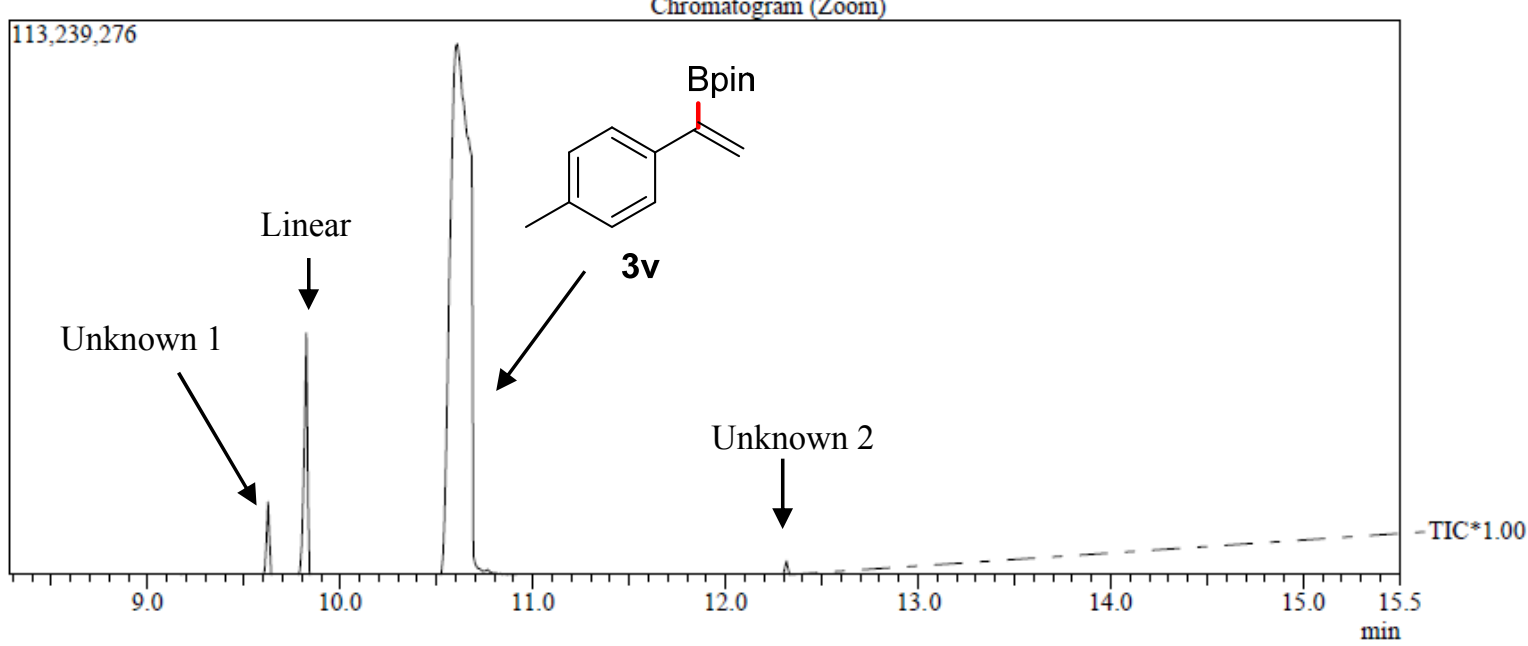

Line\#:1 R.Time:10.617(Scan\#:1274)

MassPeaks:533

RawMode:Single 10.617(1274) BasePeak:143.05(12758577)

BG Mode:None

b)

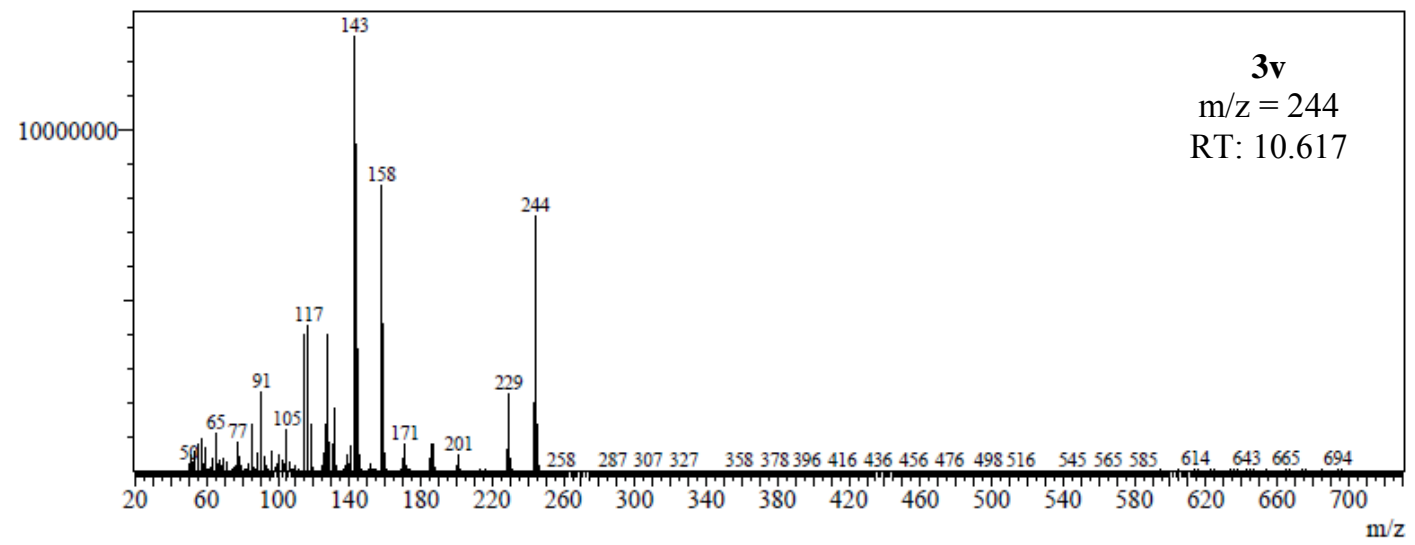

Line\#:1 R.Time:9.617(Scan\#:1154)

MassPeaks:366

RawMode:Single 9.617(1154) BasePeak:84.05(1399237)

BG Mode:Averaged 8.450-9.575(1014-1149)

c)

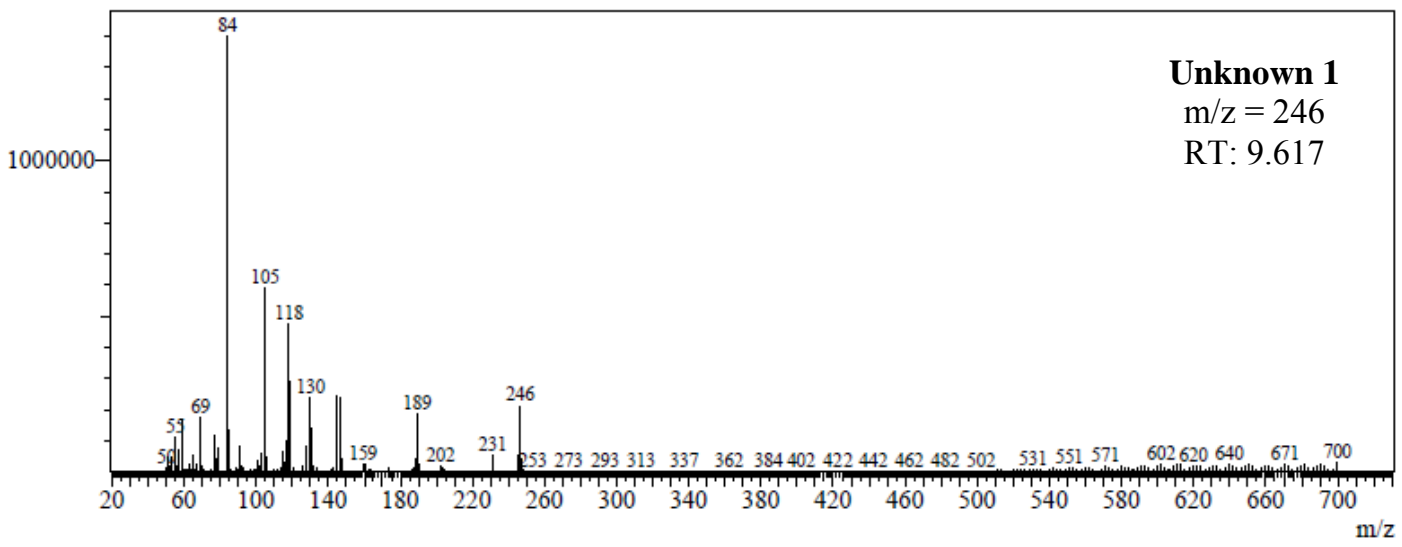


Line\#:1 R.Time:9.817(Scan\#:1178)

MassPeaks:524

RawMode:Single 9.817(1178) BasePeak:143.05(3206638)

BG Mode:None

d)

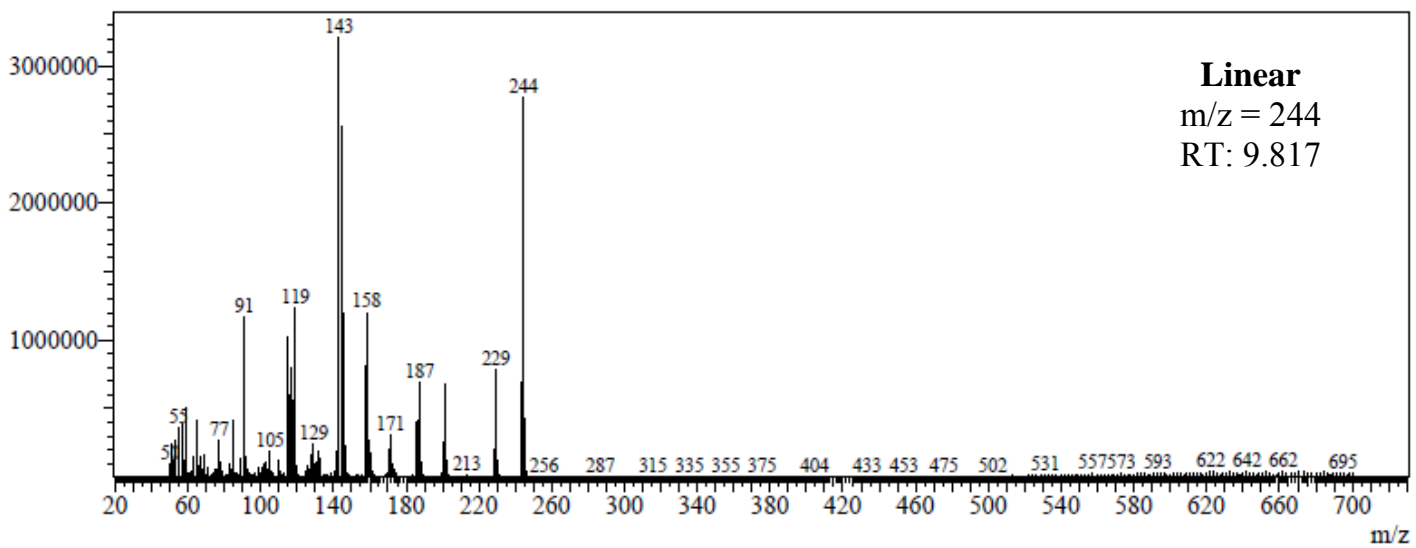

Line\#:1 R.Time:12.317(Scan\#:1478)

MassPeaks:390

RawMode:Single 12.317(1478) BasePeak:84.10(482357)

BG Mode:Averaged 10.783-12.258(1294-1471)

e)

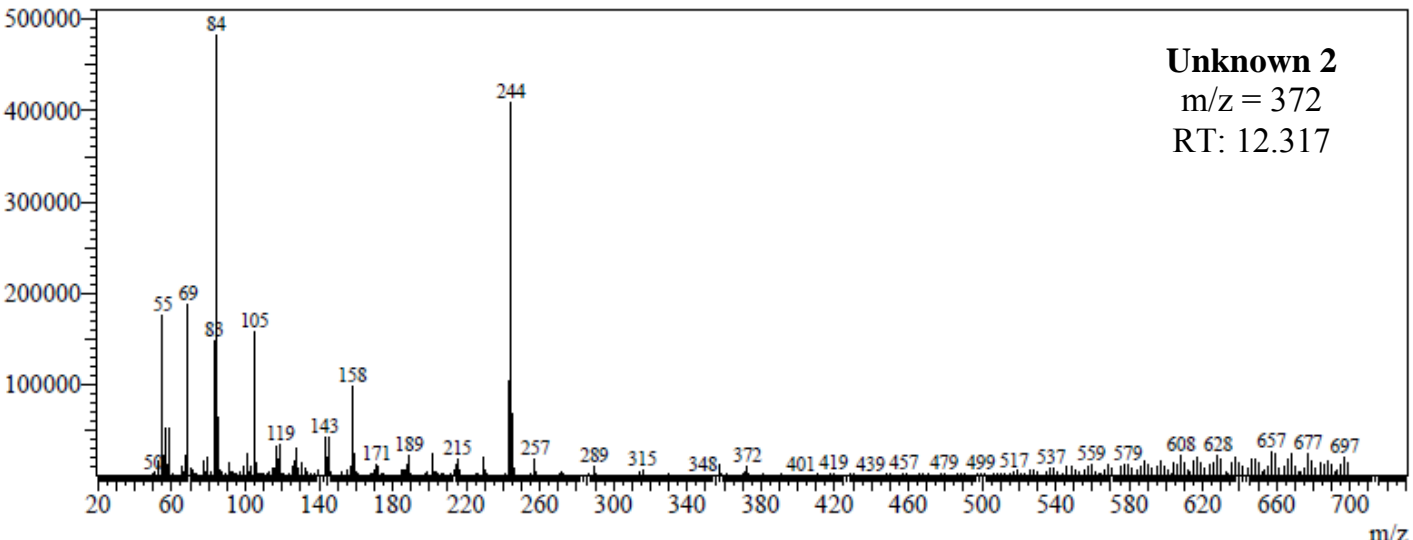

Figure S31. GC-MS chromatograms of product 3v, linear isomer, and 2 unknowns. 
Chromatogram (Zoom)

a)

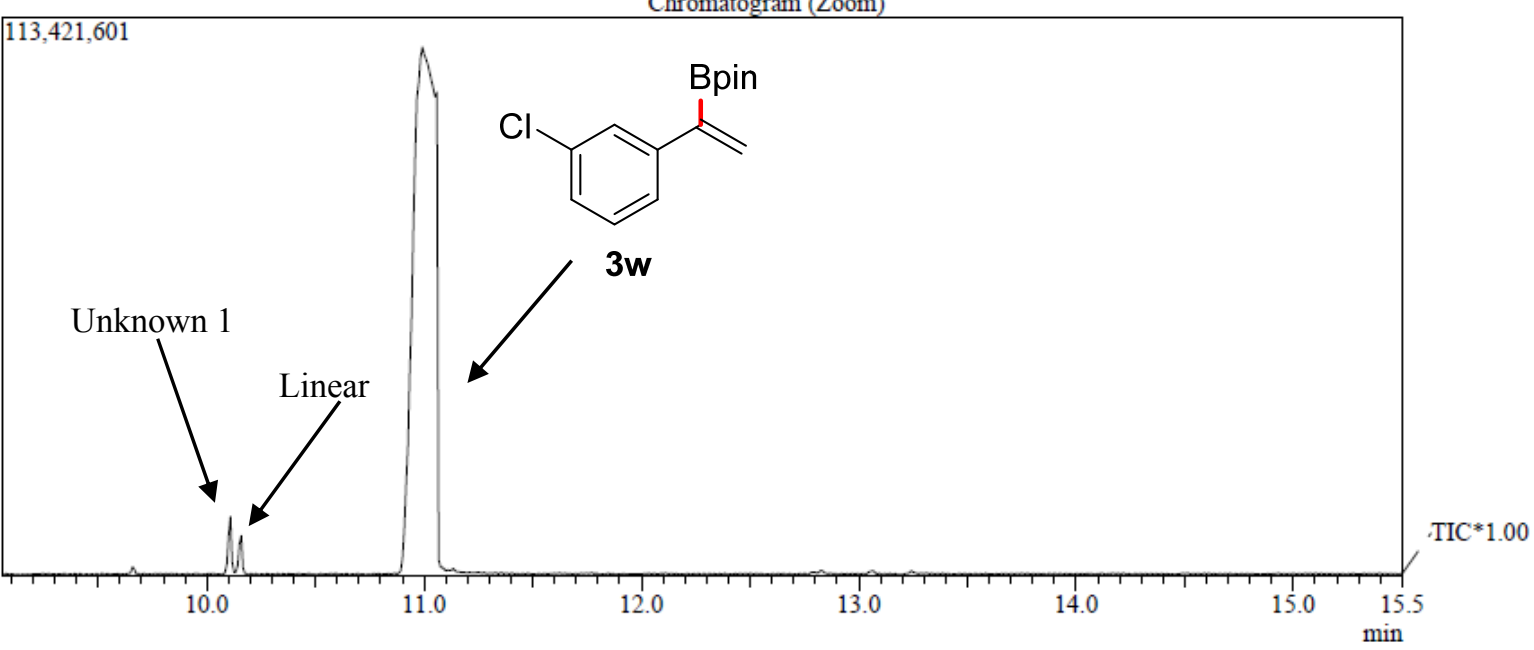

Line\#:1 R.Time:10.983(Scan\#:1318)

MassPeaks: 485

RawMode:Single 10.983(1318) BasePeak:164.10(6819146)

BG Mode:None

b)

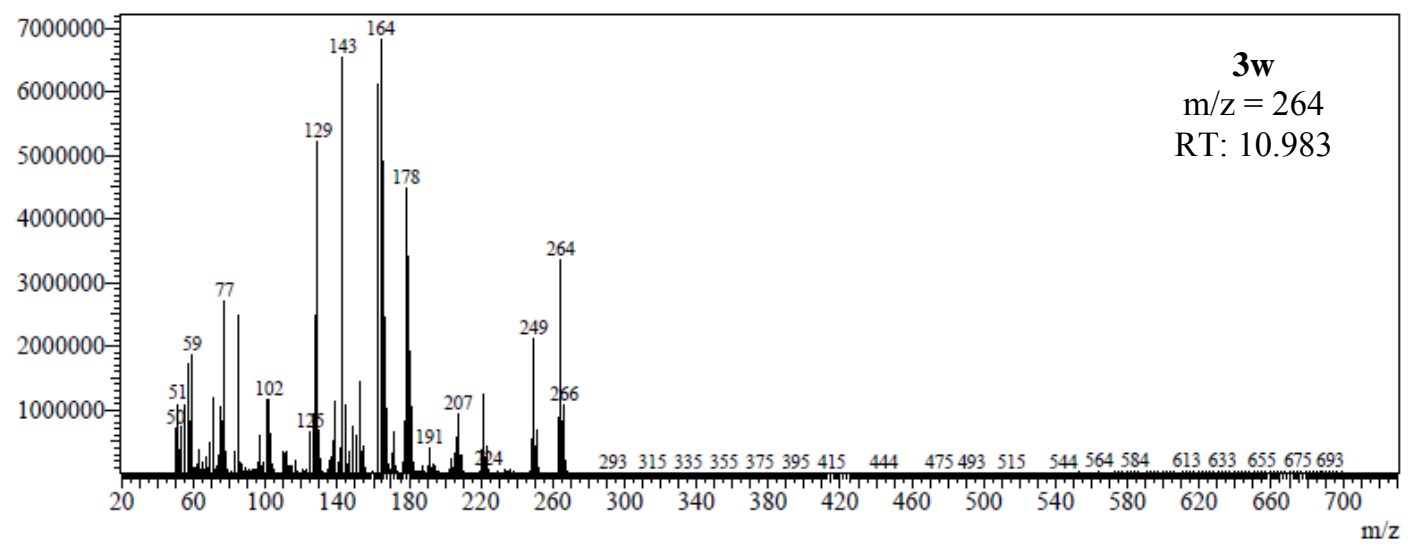

Line\#:1 R.Time:10.100(Scan\#:1212)

MassPeaks:378

RawMode:Single 10.100(1212) BasePeak:84.05(1006613)

BG Mode:Averaged 9.208-10.058(1105-1207)

c)

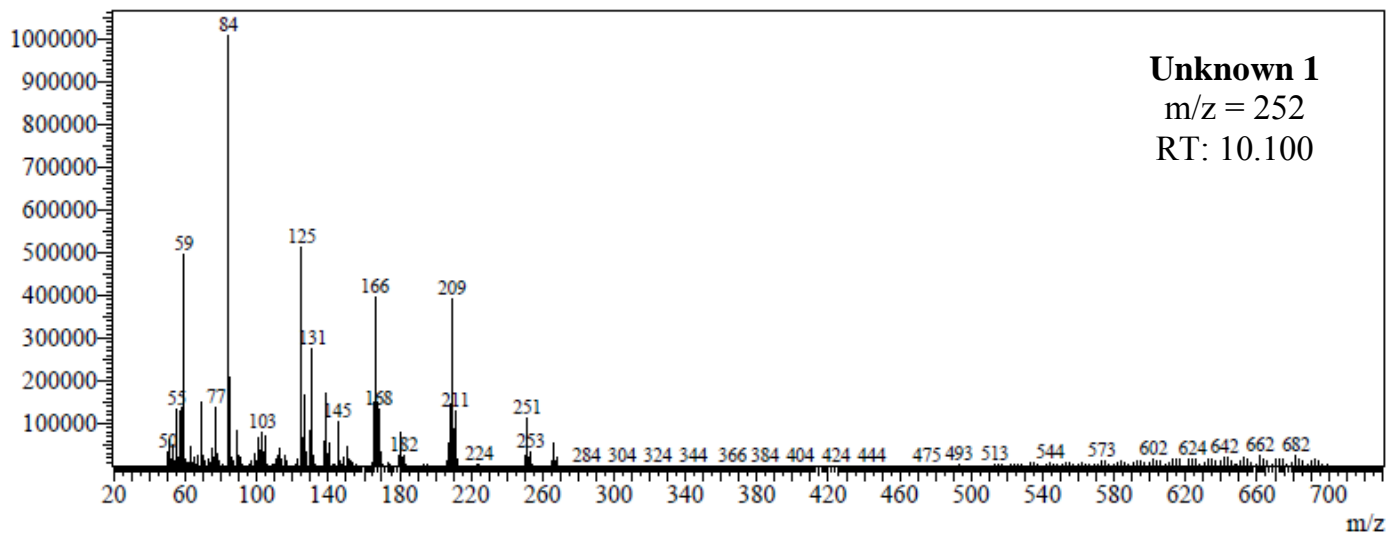


Line\#:1 R.Time:10.150(Scan\#:1218)

MassPeaks: 385

RawMode:Single 10.150(1218) BasePeak:143.05(535828)

BG Mode:Averaged 9.100-10.108(1092-1213)

d)

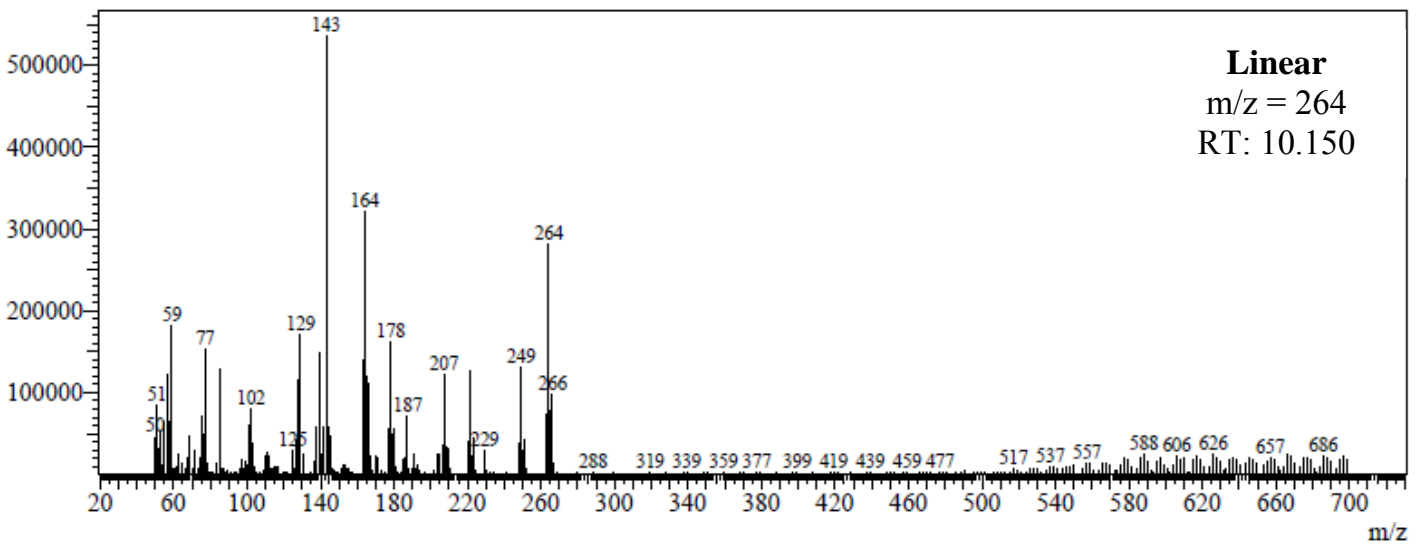

Figure S32. GC-MS chromatogram of product 3w, linear isomer, and an unknown. 


\section{References}

(1) Jafarpour, L.; Stevens, E. D.; Nolan, S. P. A Sterically Demanding Nucleophilic Carbene: 1,3-Bis(2,6Diisopropylphenyl)Imidazol-2-Ylidene). Thermochemistry and Catalytic Application in Olefin Metathesis. $J$. Organomet. Chem. 2000, 606, 49-54.

(2) Bantreil, X.; Nolan, S. P. Synthesis of N-Heterocyclic Carbene Ligands and Derived Ruthenium Olefin Metathesis Catalysts. Nat. Protoc. 2011, 6, 69-77.

(3) MacNair, A. J.; Millet, C. R. P.; Nichol, G. S.; Ironmonger, A.; Thomas, S. P. Markovnikov-Selective, Activator-Free Iron-Catalyzed Vinylarene Hydroboration. ACS Catal. 2016, 6, 7217-7221.

(4) Zhang, G.; Wu, J.; Li, S.; Cass, S.; Zheng, S. Markovnikov-Selective Hydroboration of Vinylarenes Catalyzed by a Cobalt(II) Coordination Polymer. Org. Lett. 2018, 20, 7893-7897.

(5) Peng, J.; Docherty, J. H.; Dominey, A. P.; Thomas, S. P. Cobalt-Catalysed Markovnikov Selective Hydroboration of Vinylarenes. Chem. Commun. 2017, 53, 4726-4729.

(6) Do, N. M.; Olivier, M. A.; Salisbury, J. J.; Wager, C. B. Application of Quantitative 19 F and 1 H NMR for Reaction Monitoring and In Situ Yield Determinations for an Early Stage Pharmaceutical Candidate. Anal. Chem. 2011, 83, 8766-8771.

(7) Lennox, A. J. J.; Nutting, J. E.; Stahl, S. S. Selective Electrochemical Generation of Benzylic Radicals Enabled by Ferrocene-Based Electron-Transfer Mediators. Chem. Sci. 2018, 9, 356-361.

(8) Li, H.; Wang, L.; Zhang, Y.; Wang, J. Transition-Metal-Free Synthesis of Pinacol Alkylboronates from Tosylhydrazones. Angew. Chemie Int. Ed. 2012, 51, 2943-2946.

(9) Huang, J.; Yan, W.; Tan, C.; Wu, W.; Jiang, H. Palladium-Catalyzed Regioselective Hydroboration of Aryl Alkenes with B 2 Pin 2. Chem. Commun. 2018, 54, 1770-1773.

(10) Hu, J.; Sun, H.; Cai, W.; Pu, X.; Zhang, Y.; Shi, Z. Nickel-Catalyzed Borylation of Aryl- and Benzyltrimethylammonium Salts via C-N Bond Cleavage. J. Org. Chem. 2016, 81, 14-24.

(11) Ng, E. W. H.; Low, K.-H.; Chiu, P. Synthesis and Applications of Unquaternized C-Bound Boron Enolates. J. Am. Chem. Soc. 2018, 140, 3537-3541.

(12) Guan, W.; Michael, A. K.; McIntosh, M. L.; Koren-Selfridge, L.; Scott, J. P.; Clark, T. B. Stereoselective Formation of Trisubstituted Vinyl Boronate Esters by the Acid-Mediated Elimination of $\alpha$-Hydroxyboronate Esters. J. Org. Chem. 2014, 79, 7199-7204.

(13) Zhang, P.; Meijide Suárez, J.; Driant, T.; Derat, E.; Zhang, Y.; Ménand, M.; Roland, S.; Sollogoub, M. Cyclodextrin Cavity-Induced Mechanistic Switch in Copper-Catalyzed Hydroboration. Angew. Chem. Int. Ed. 2017, 56, 10821-10825.

(14) Claudel, S.; Gosmini, C.; Paris, J. M.; Périchon, J. A Novel Transmetallation of Arylzinc Species into Arylboronates from Aryl Halides in a Barbier Procedure. Chem. Commun. 2007, No. 35, 3667.

(15) Farnaby, W.; Fieldhouse, C.; Hazel, K.; Kerr, C.; Kinsella, N.; Livermore, D.; Merchant, K.; Miller, D. PCT Int. Appl. (2013), WO 2013027000 A1 20130228.

(16) Hawkeswood, S.; Stephan, D. W. Syntheses and Reactions of the Bis-Boryloxide O(Bpin $)_{2}\left(\mathrm{Pin}=\mathrm{O}_{2} \mathrm{C}_{2} \mathrm{Me}_{4}\right)$. Dalton Trans. 2005, 2182-2187. 